Aus der Klinik für Psychiatrie und Psychotherapie

(Komm. Leiter: Prof. Dr. med. B. Bandelow) im Zentrum Psychosoziale Medizin

der Medizinischen Fakultät der Universität Göttingen

\title{
Neuronale Korrelate von Placeboeffekt, Furchtextinktion und willentlicher Emotionsregulation
}

Eine Metaanalyse über die Regulation negativer Gefühle

\author{
INAUGURAL-DISSERTATION \\ zur Erlangung des Doktorgrades \\ der Medizinischen Fakultät \\ der Georg-August-Universität zu Göttingen
}

vorgelegt von

Katharina Geier

aus

Minden

Göttingen 2013 
Dekan:

1. Berichterstatter:

2. Berichterstatterin:

3. Berichterstatter:
Prof. Dr. rer. nat. H. K. Kroemer

Prof. Dr. med. O. Gruber

Prof. Dr. rer nat. M. Wilke

Prof. Dr. med. M. Oppermann

Tag der mündlichen Prüfung: 12.08.2014 


\section{Inhaltsverzeichnis}

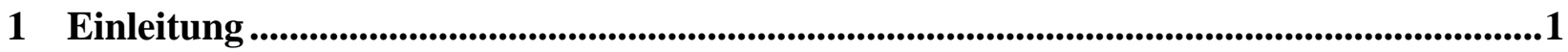

1.1 Kurzer Einblick in die Furchtextinktion................................................................................ 1

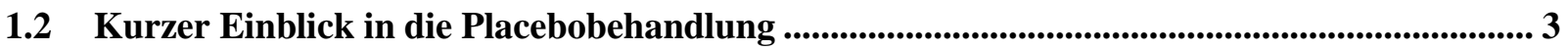

1.3 Kurzer Einblick in die willentliche Emotionsregulation.........................................................5

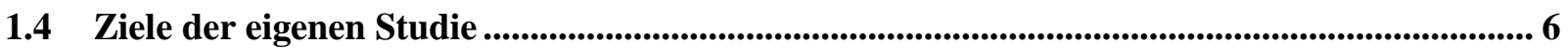

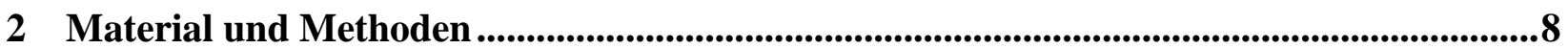

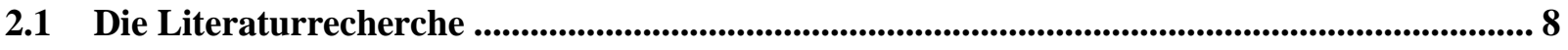

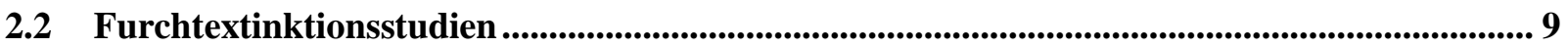

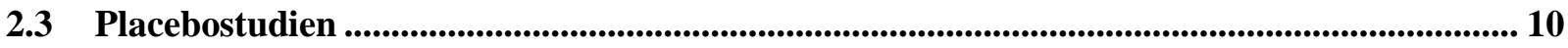

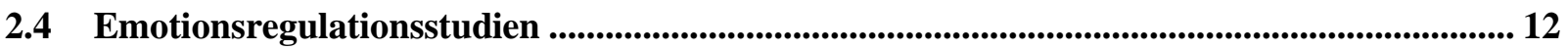

2.5 Koordinatenselektion .............................................................................................................................. 13

2.6 Die Metaanalyse....................................................................................................................................... 14

2.7 Activation likelihood estimation (ALE) ....................................................................................... 15

2.8 Durchführung der ALE-Metaanalyse .............................................................................................. 16

2.9 Konkretes Vorgehen bei Durchführung der Metaanalyse............................................................. 17

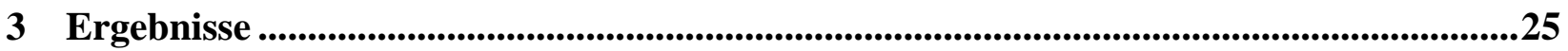

3.1 VMPFC-Aktivität während der Regulation negativer Emotionen .........................................25

3.2 Regionen mit verstärkter Aktivität neben dem VMPFC .................................................... 27

3.3 Gehirnaktivierungen bei der Emotionsregulation - Beschränkung auf Studien mit ähnlicher

Verteilung an weiblichen und männlichen Probanden.............................................................. 31

3.4 Zentrum der Überlappung von Aktivität............................................................................................ 32

3.5 Paarmetaanalyse mit Emotionsregulations- und Placebostudien .......................................... 32

3.6 Amygdala-Deaktivierung während der Reduktion negativer Emotionen............................... 35

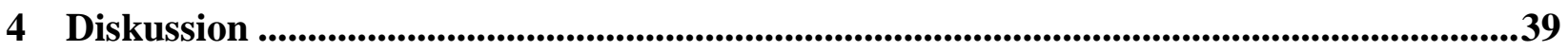

4.1 Ziele und Überblick über die wichtigsten Ergebnisse dieser Arbeit..................................... 39

4.2 Der VMPFC: Anatomie, Einflüsse und Funktionen ................................................................ 41

4.2.1 Studientypübergreifende VMPFC-Aktivität während der Regulation negativer Emotionen. 44

4.2.2 Weitere Erklärungsmodelle für die VMPFC-Aktivierung ........................................... 45

4.2.2.1 Veränderte Wahrnehmung durch Erwartungsmanipulation .............................................................. 46

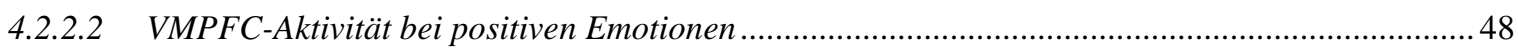

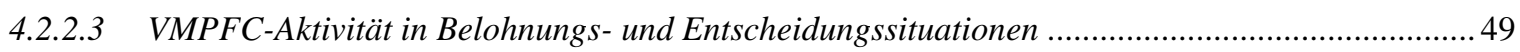

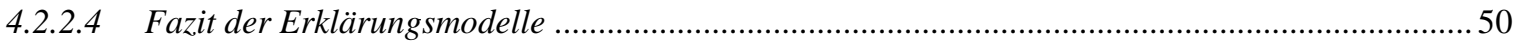


4.2.3 Inkludierte Studien der Metaanalyse ohne VMPFC-Aktivität ........................................... 50

4.3 Weitere Gehirnaktivierungen während der Regulation negativer Emotionen...................... 53

4.3.1 Weitere Gehirnaktivierungen in Emotionsregulationsstudien ........................................ 54

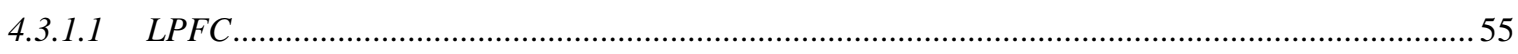

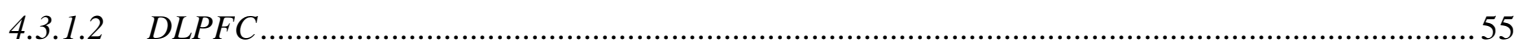

4.3.1.3

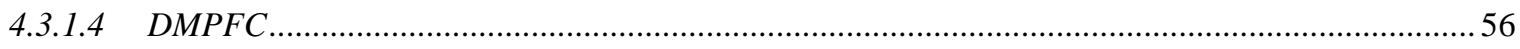

4.3.1.5 Parietaler Kortex, temporaler Kortex, inferiorer frontaler Gyrus............................................... 57

4.3.2 Weitere Gehirnaktivierungen in Placebostudien ........................................................ 57

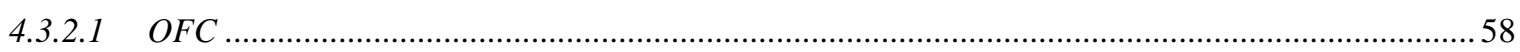

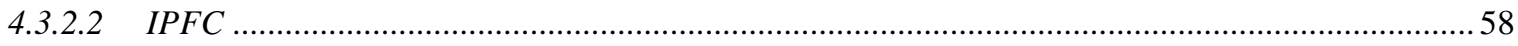

4.3.3 Weitere Gehirnaktivierungen in Furchtextinktionsstudien ........................................... 59

4.3.4 Paarmetaanalyse von Emotionsregulations- und Placebostudien.................................... 59

4.3.4.1 ACC

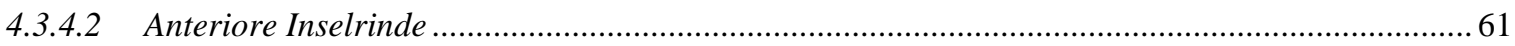

4.4 Die Amygdala - Zentrum der Deaktivierung während Regulation negativer Emotionen .... 62

4.4.1 Die Amygdala: Kurzer Einblick in Einfluss und Funktion ........................................... 63

4.4.2 Inkludierte Studien der Metaanalyse ohne Amygdala-Deaktivität................................... 63

4.5 Grenzen dieser Metaanalyse................................................................................................................... 64

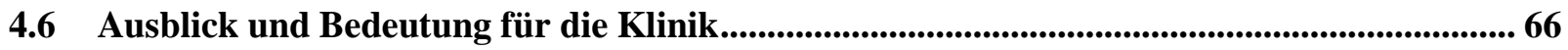

4.6.1 Klinische Relevanz der Placebostudien dieser Metaanalyse .......................................... 67

4.6.2 Klinische Relevanz der Emotionsregulationsstudien dieser Metaanalyse .......................... 68

4.6.3 Klinische Relevanz der Furchtextinktionsstudien dieser Metaanalyse ............................. 68

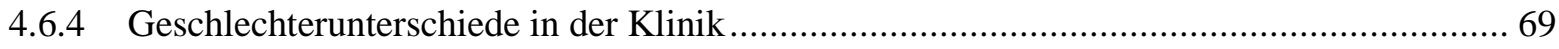

$5 \quad$ Zusammenfassung ............................................................................................................................70

6 Literaturverzeichnis.........................................................................................................................72

$7 \quad$ Abbildungsverzeichnis .......................................................................................................................97

8 Tabellenverzeichnis............................................................................................................................98

$8.1 \quad$ Ergänzende Tabellen........................................................................................................................ 99

9 Abkürzungsverzeichnis........................................................................................................................101

10 Publikation zum Dissertationsthema.............................................................................................103 


\section{$1 \quad$ Einleitung}

„Die Gefühlsregulation erfolgt mit Hilfe der Verarbeitung im Gehirn, durch die wir beeinflussen, welche Gefühle wir haben, wann wir sie haben und wie wir sie wahrnehmen und ausdrücken“ (Gross 1998, S. 275).

Diese Annahme war Ausgangspunkt unterschiedlicher Studien, die sich in den letzten Jahren mit dem Thema „emotionale Regulation“ beschäftigten.

Es wurde in unterschiedlichen Ansätzen untersucht, wie der Mensch die Wahrnehmung eines angsteinflößenden Ereignisses verändern kann und gleichzeitig bemühte man sich, die damit im Zusammenhang stehenden neurophysiologischen und neurochemischen Prozesse im Gehirn aufzuzeigen (Diekhof et al. 2011a, S. 275). Diese Untersuchungen waren von besonderem klinischen Interesse, da die Fähigkeit, Emotionen zu regulieren, insbesondere bei der Bewältigung negativer Ereignisse (Eippert et al. 2007, S. 409) entscheidend für die geistige Gesundheit ist. Angststörungen zählen zu den häufigsten psychischen Erkrankungen. Die fehlende Möglichkeit zur Angstlöschung führt beispielsweise zu psychischen Störungen wie Posttraumatische Belastungsstörungen (Milad et al. 2006). Generell ist es ein Aspekt des persönlichen Wohlbefindens und einer erfolgreichen sozialen Interaktion, Gefühle nicht immer vollständig zum Ausdruck zu bringen, sondern Emotionen situationsabhängig kontrollieren zu können (Gross 2002).

Es gibt drei unterschiedliche Studientypen, die sich in besonderer Weise mit der Regulation von Emotionen auseinandersetzen. Dazu zählen Studien über die Furchtextinktion, über die Placebobehandlung sowie über die willentliche Emotionsregulation bzw. emotionale Neubewertung.

Das Versuchsdesign und der theoretische Hintergrund der drei unterschiedlichen Studientypen stellen sich wie folgt dar.

\subsection{Kurzer Einblick in die Furchtextinktion}

Das Löschen der Angst zu lernen ist wichtig für ein adaptives Verhalten in unserer sich ständig verändernden Umwelt (Kalisch et al. 2006b, S. 9503). 
Es gibt zwei Formen der Furchtextinktion. Zum einen gibt es die Pavlovsche Löschung, das heißt das Löschen einer klassisch konditionierten Antwort, wenn der konditionierte Reiz nicht länger gepaart mit dem unkonditionierten Stimulus dargeboten wird (Pavlov 1927), auf die im Folgenden noch genauer eingegangen wird. Zum anderen gibt es die operante Extinktion, bei der eine konditioniert operante Antwort gelöscht wird, wenn der Antwort nicht mehr länger ein verstärkender Stimulus wie eine Belohnung folgt, oder eine Bestrafung durch Hinzufügen eines aversiven Reizes erfolgt.

Die meisten Studien der hier durchgeführten Metaanalyse zum Thema „Furchtextinktion“ orientieren sich am Pavlovschen Prinzip der Konditionierung. Die Grundlage ist das Verständnis über die Konditionierung, da Konditionierungsprozesse wichtig für die Entwicklung und Aufrechterhaltung von Angststörungen sind (Koler 2006, S.7).

Konditionierung bedeutet das Lernen der Assoziation zwischen einem konditionierten Reiz (CS) und einem unkonditioniertem Reiz (UCS) (Koler 2006, S. 7). Ein unbedingter oder unkonditionierter Reiz (UCS: z.B. ein Stromstoß) führt zu einer unbedingten oder unkonditionierten Reaktion (UCR: erzeugte Angstreaktion durch den leichten elektrischen Schock). Beim pavlovschen Konditionieren wird ein neutral bedingter Reiz (NS) - z.B. ein Ton mit einem aversiven unbedingten Reiz (UCS), z.B. einem leichten elektrischem Schock, gepaart. Nach mehreren Wiederholungen und in Folge des Lernprozesses wird der ursprünglich neutrale Reiz (NS) zu einem bedingten Reiz (CS) und führt allein eine erlernte angepasste bedingte Reaktion herbei (CR: Angst bei elektrischem Schock) (Corcoran und Quirk 2007).

Bei der Löschung bzw. Extinktion wird der bedingte Reiz (CS) für längere Zeit allein dargeboten (ohne den UCS), sodass allmählich die bedingte Reaktion (CR) verschwindet. Danach existieren zwei Erinnerungen im Gehirn. Zum einen der CS/UCS-Verband für das Konditionieren/Lernen (,Angstgedächtnis“) und der CS/Kein-UCS-Verband für das „Löschgedächtnis“ (Milad et al. 2007, Kalisch et al. 2006b).

Zusätzlich wird die Löschung in zwei Phasen eingeteilt. Die erste ist das Erlernen der Furchtextinktion, die zweite die Festigung bzw. die Entwicklung eines Löschgedächtnisses (Lang et al. 2009).

Die Furchtextinktion beinhaltet die Bildung eines neuen Gedächtnisses, das den alten konditionierten Pfad hemmt, jedoch ohne ihn zu löschen (Myers und Davis 2007). Dieses neue 
Löschgedächtnis konkurriert mit dem alten Angstgedächtnis um die Kontrolle über das Verhalten (Bouton 2004, Delamater 2004, Myers und Davis 2002). Das Löschgedächtnis ist jedoch labil und schwach verglichen mit der konditionierten Angst (Akirav und Maroun 2007).

So kann das Angstgedächtnis in bestimmten Situationen jederzeit wieder aktiviert werden, sodass die Wahrscheinlichkeit, dass ein richtiger Gefahrenhinweis missachtet wird, reduziert wird. Dies ist natürlich wichtig, da das Fehlen dieser Funktion möglicherweise lebensgefährlich sein könnte (Kalisch et al. 2006b).

\subsection{Kurzer Einblick in die Placebobehandlung}

Bei der Darbietung eines aversiven Reizes in Kombination mit einem Placebo wird die Angstabschwächung oder Schmerzlinderung durch Täuschung oder irreführende Hinweise und damit verbundene veränderte Erwartungen erzeugt. Die Placebos lassen den Versuchsprobanden glauben, dass ein schmerzlindernder Effekt eintritt. Dieses Ergebnis wird durch zwei Prozesse erzielt (Diekhof et al. 2011a, S. 276). Der Placeboeffekt hängt somit einerseits von der positiven Erwartungshaltung gegenüber dem Erfolg der Behandlung und der analgetischen Wirkung ab und andererseits von der Konditionierung (Amanzio und Benedetti 1999, S. 484).

Das bedeutet, dass allein die Erwartung eines weniger schmerzhaften Reizes als wirklich dargeboten die spinale nozizeptive Verarbeitung beeinflusst (Goffaux et al. 2007, Matre et al. 2006) und somit die Wahrnehmung des Reizes zum „Positiven“ verändert (Diekhof et al. 2011a, S. 276). Die Größe der Erwartungshaltung bezüglich der Wirksamkeit der Behandlung setzt sich aus Faktoren wie der individuell grundsätzlichen Einstellung zu der Placebobehandlung, dem Ruf des Behandlers, der Art der Placebobehandlung (Injektion oder orale Placebogabe (Zhang et al. 2008)), der Größe, Farbe und dem Preis des Placebos (Waber et al. 2008) zusammen. Ebenso ist der Placeboeffekt abhängig von den Erwartungen, die entweder durch experimentelle Manipulation (Montgomery und Kirsch 1997, Price et al. 1999, Voudouris et al. 1989, Voudouris et al. 1990) oder Opiat-Gabe induziert werden können.

Diese zweite Möglichkeit, Erwartungen über eine Schmerzreduktion zu erzeugen, funktioniert durch zunächst produzierte Analgesie durch wirksame Medikamente wie Opiate und anschließende Verabreichung eines Placebos, mit der fehlerhaften Information, es handele sich um ein Opiat 
(Amanzio und Benedetti 1999). „Der Placeboerfolg ist somit entscheidend abhängig von dem Lerneffekt“" (Petrovic et al. 2005, S. 957). Ein opioides Medikament in oraler Applikationsform einer Pille als UCS induziert bei dem Probanden als UCR Schmerzfreiheit. Schließlich erzeugt die Placebopille als CS ebenso eine Reduktion der Schmerzen (CR).

Plassmann et al. (2008) (S. 1050) behaupten, dass die „erfahrene Annehmlichkeit“ als Lernsignal funktioniert, das dem Gehirn als Leitfaden für spätere Entscheidungen in der Zukunft dient. Es könnte dazu führen, dass man Situationen in der Zukunft positiv beurteilt, wenn man gelernt hat, Dinge positiv zu bewerten (z.B. die Wahrnehmung eines reduzierten Schmerzes oder Angstreduktion). Dies könnte als therapeutischer Ansatz bei der Behandlung von Krankheiten wie Depressionen dienen.

Zusätzlich wurde gezeigt, dass die Vorahnung und Erwartung von reduziertem Schmerz und die aktuelle Wahrnehmung von einer Schmerzverringerung als Basis eines selbstverstärkenden Rückkopplungskreislaufes dienen könnte, der durch vorheriges Lernen unterstützt wird (Watson et al. 2009). Eine Placebobehandlung wird danach erst richtig effektiv, wenn man mit Hilfe des Konditionierens ein Gedächtnis schafft mit Informationen über die Wirkung von Placebos. Durch eintreffende sensorische Informationen muss das Gedächtnis immer wieder erneuert und angepasst werden (Rolls 2000).

Pharmakologische Studien zeigten, dass Placeboanalgesie durch den Opioid-Antagonist Naloxon antagonisiert werden kann. Dies bedeutet, dass Placeboanalgesie auf das Opioidsystem Einfluss hat (Benedetti et al. 1999). Das Opioidsystem des Menschen ist direkt in die Verarbeitung und Regulation von Emotionen involviert (Liberzon et al. 2002, Zubieta et al. 2003). Naloxon blockiert den subjektiven Placeboeffekt jedoch nicht komplett (Eippert et al. 2009). Dies lässt vermuten, dass es noch eine weitere nicht-opioide Komponente gibt (Amanzio und Benedetti 1999, Gracely et al. 1983, Grevert et al. 1983, Vase et al. 2005).

Ein weiteres System, das bei der emotionalen Placeboanalgesie beteiligt ist, ist das Dopaminsystem (Petrovic et al. 2005). Auch Tierstudien haben gezeigt, dass bei der Erwartung eines positiven Ergebnisses die mesolimbischen Zellen Dopamin vermehrt ausschütten (Tobler et al. 2005). Bei der Placeboanwendung werden also zum einen Dopamintransmitter ausgeschüttet, die Dopaminrezeptoren D2/D3 in ventralen basalen Ganglien beeinflussen, und zum anderen Opioide, die Opioid-Rezeptoren im rostralen subgenualen Gyrus cinguli, orbitofrontalen Kortex, anteriorer 
und posteriorer Insel, medialen Thalamus, Nucleus accumbens, Amygdala und PAG beeinflussen (Scott et al. 2008, S. 229).

\subsection{Kurzer Einblick in die willentliche Emotionsregulation}

„Gefühle haben Einfluss auf die Aufmerksamkeit (Vuilleumier 2005), das Treffen von Entscheidungen (Bechara et al. 1999), das Gedächtnis (Phelps 2006), physiologische Reaktionen (Cacioppo et al. 2000, Levenson 2003) und soziale Interaktionen (Keltner und Kring 1998)“ (Goldin et al. 2007, S. 1). Die Fähigkeit, Situationen emotional neu zu bewerten, ist daher wichtig für ein erfolgreiches Agieren im alltäglichen Leben.

Durch die willentliche Kontrolle über die Wahrnehmung negativer Emotionen, beispielsweise von Angst, wird eine objektive Sicht auf negative Ereignisse ermöglicht. Mit Hilfe von Neubewertung kann die Wahrnehmung des negativen Ereignisses verändert werden und das Ausmaß der Angst reduziert werden. Die Methoden der Neubewertung umfassen das emotionale Distanzieren von einem negativen Ereignis, sowie die Neuinterpretation, die Betrachtung des negativen Ereignisses als ,unecht““ oder das Vorstellen von positiv assoziierten Bildern.

Man vermutet, dass auf neuronaler Ebene die Angstregulation, wie im Folgenden dargestellt, funktioniert.

Kalisch et al. 2006c (S. 1273) nehmen an, dass bei „der emotionalen Regulation im Arbeitsgedächtnis ein Austausch (Bower 1981) von angstverbundenen mentalen Inhalten zu unängstlichem Material, so genanntes ,sicheres“ oder „gesichertes“, erfolgt, was zur Abschaltung des Angstnetzwerkes führt und somit weniger Angst empfunden wird.

Eine generelle Deaktivierung des Angstnetzwerkes durch Austausch oder Ersatz kann die oft beobachtete Abschwächung der autonomen Reaktion während der Emotionsregulation erklären (Kalisch et al. 2005, Gross 2002, Jackson et al. 2000). Wie auch in den anderen zwei Studientypen wird hier an eine Form der Konditionierung erinnert. Durch emotionale Regulationen, kommt es bei der nächsten Situation, in der eine Emotionsregulation erforderlich ist, nur noch zu einer abgeschwächten Anstrengung während der Regulation.

Diese Art der Reduktion negativer Gefühle durch Emotionsregulation ist, im Vergleich zu den anderen Arten der Gefühlsregulierung durch die Placebobehandlung oder die Furchtextinktion, 
anspruchsvoller und erfordert eine willentliche Kontrolle, um mit angsterzeugenden Situationen zurecht zu kommen (Diekhof et al. 2011a, S. 276).

\subsection{Ziele der eigenen Studie}

Für diese Metaanalyse waren Studien über die Furchtextinktion (Fear extinction), über den Placeboeffekt und zum dritten für Studien über die willentliche Emotionsregulation und Neubewertung (Reappraisal) von besonderem Interesse. In allen drei Studientypen wurden die Probanden einem aversiven Reiz ausgesetzt. Bei den Studien mit Placebobehandlung und bei den Studien über Furchtextinktion waren es beispielsweise ungefährliche Stromstöße, bei den Emotionsregulationsstudien waren es teilweise angsterzeugende Bilder. Gemeinsam war außerdem, dass in allen drei Studienbereichen versucht wurde, durch unterschiedliche Formen der Gefühlsregulation, negative Emotionen (im Besonderen Angst) zu reduzieren.

Das Ziel dieser Untersuchung war herauszufinden, ob in allen drei experimentellen Studienbereichen ähnliche neuronale Gehirnstrukturen zur Kontrolle der wahrgenommenen negativer Emotionen aktiviert wurden, und die Gemeinsamkeiten in der Gehirnaktivierung herauszuarbeiten. Es wurde die Untersuchungshypothese aufgestellt, dass die aktivierten Gehirnareale, die in allen drei unterschiedlichen Studientypen gefunden wurden, als Teil eines allgemeingültigen Regulationszentrums von negativen Emotionen betrachtet werden können, da ja die Reduktion der negativen Emotionen das in allen Studien gemeinsame Ergebnis war. Deaktivierungen in den Studien waren folglich wichtig für die Wahrnehmung negativer Emotionen. Zusätzlich stellte sich die Frage, ob bei den geistig anspruchsvolleren emotionalen Regulationsstrategien, wie bei der willentlichen Emotionsregulation und der Placebobehandlung, weitere Gehirnregionen für eine erfolgreiche Reduktion negativer Emotionen von Bedeutung sind.

Das Herausarbeiten der aktivierten Gehirnregionen war nicht nur ein wichtiger Aspekt zum Verständnis des neuronalen Mechanismus der Regulation negativer Emotionen wie der Angst, sondern auch um herauszufinden, wie mentale Prozesse generell bestimmte psychologische Reaktionen hervorrufen und die Wahrnehmung beeinflussen können (Diekhof et al. 2011a, S. 276). 
Die Auswertung der unterschiedlichen Studientypen mit Hilfe der koordinatenbasierten Metaanalyse sollte Gehirnregionen herausarbeiten, die bei der Reduktion negativer Emotionen beteiligt sind.

Dabei vergleicht die koordinatenbasierte quantitative Metaanalyse die relevanten bildgebenden Studien miteinander und bietet so die Möglichkeit, Übereinstimmungen von Ergebnissen von verschiedenen experimentellen Studien aufzuzeigen (Laird et al. 2005a). Dadurch ist es möglich Ergebnisse zu generalisieren (Laird et al. 2009b, S. 7), die unabhängig von unterschiedlichen experimentellen Ausführungen, Datenanalysetechniken und dem ungenauen Gebrauch anatomischer Bezeichnungen sind, welche sonst Vergleiche verkomplizieren könnten (Caspers et al. 2010). 


\section{Material und Methoden}

Im Folgenden werden die Ergebnisse einer koordinatenbasierten quantitativen Metaanalyse mit der Activation-likelihood-estimation (ALE)-Methode von neurologischen Studien präsentiert, die bildgebende Verfahren der funktionellen Magnetresonanztomographie (fMRI) oder der Positronen-Emissions-Tomographie (PET) benutzt haben, um die Gehirnreaktionen bei willentlicher Emotionsregulation, dem Placeboeffekt und bei der Furchtextinktion zu zeigen.

Durch die Betrachtung der Gehirnaktivierung in den verschiedenen Studientypen sollte die Funktion von Gehirnregionen bei der Reduktion negativer Emotionen aufzeigt werden.

Gemeinsam war in allen drei Versuchstypen, dass die Probanden einem aversiven Reiz ausgesetzt wurden und ihre dadurch erzeugten Gefühle regulieren sollten. Die Versuchstypen präsentierten unterschiedliche Möglichkeiten der Regulation negativer Emotionen. „Emotionale Regulation kann beabsichtigt oder habituell, bewusst oder unbewusst sein und Veränderungen im Ausmaß, in der Dauer oder in der Qualität von einem oder mehreren Bestandteilen einer Gefühlsreaktion beinhalten“" (McRae et al. 2008, S. 146).

\subsection{Die Literaturrecherche}

Die Literatursuche wurde mit der Internet-Suchmaschine PubMED durchgeführt. Um Emotionsregulationsstudien zu selektieren, wurden die Suchbegriffe „emotion regulation“ oder „reappraisal“ gewählt, jeweils kombiniert mit einem Plussymbol $(,,+$ “) oder „AND“ und den Stichwörtern „fMRI“ oder „PET“ (z.B.: ,emotion regulation AND fMRI“). Eine ähnliche Suchweise wurde bei den anderen beiden Studientypen angewandt. Für das Sammeln der Furchtextinktionsstudien wurden die Suchbegriffe „fear extinction“ oder ,extinction learning“ benutzt und diese jeweils wieder mit „,+“ oder „AND“ und den Stichwörtern „fMRI“ oder „PET“

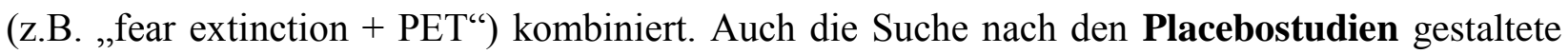
sich auf diese Weise, bei der das Wort „placebo“ zusammen mit „,+“ oder „AND“ und der Stichwörtern „fMRI“ oder „PET“ in die PubMED Suchmaschine eingegeben wurde (z.B. ,placebo AND fMRT"). 
Die Suche wurde begrenzt auf die relevanten Artikel, die im Zeitraum 2000 bis Juni 2010 herausgegeben wurden. Zusätzlich wurde noch die Studie Diekhof et al. (2011b) miteinbezogen, die zu dem Zeitpunkt der Suche noch nicht veröffentlicht war. Es wurde sich auf funktionelle Studien mit neurologischen bildgebenden Verfahren konzentriert, die gesunde Erwachsene untersuchten.

Studien mit Probanden, die neurologische Krankheiten aufwiesen, oder mit Probanden unter Medikamenteneinnahme wurden ausgeschlossen. Auch Studien, die von pädiatrischen oder Jugendfällen handelten, wurden der systematischen Literaturüberprüfung nicht beigefügt. Ebenso wurden Studien mit Tierversuchen nicht berücksichtigt. Ferner wurden Studien ausgeschlossen, sofern sie nur von anatomischen Regionen ohne Koordinaten berichteten (z. B. Benedetti et al. 2003, Benedetti et al. 2005, Holmes et al. 2004, Mathews 2004, Phelps 2006, Ploghaus et al. 2003, Rainville 2002, Sander et al. 2005, Sotres-Bayon et al. 2004, Sotres-Bayon et al. 2006, Wager 2005).

Anfangs zeigten sich nach erster Prüfung der Literatur 173 relevante Emotionsregulationsstudien, 43 Placebostudien und 25 Fearextinktionstudien. Die Anzahl wurde jedoch nach Anwendung der Ausschlusskriterien stark reduziert. Insgesamt wurden neun bildgebende Studien zum Thema „Furchtextinktion“(Tabelle 2.1), 13 zum „Placeboeffekt“ (Tabelle 2.2) und 23 Artikel zum Thema „willentliche Emotionsregulation“ (Tabelle 2.3) ausgewählt.

\subsection{Furchtextinktionsstudien}

Während der Furchtextinktion wird die zuvor konditionierte Angstantwort (CR) gelöscht durch das Fehlen der Verstärkung des angstkonditionierenden Reizes (CS) durch den unkonditionierten Reiz (UCS). Danach existieren zwei Erinnerungen im Gehirn. Zum einen gibt es den CS/UCS- Verband für das konditioniete Angstgedächtnis und zum zweiten den CS/ Kein-UCS-Verband für das Löschgedächtnis (Milad et al. 2007, S. 446). Das Angstgedächtnis wird während des Angstlöschung nicht gelöscht, sondern lediglich gehemmt, sodass die Wahrscheinlichkeit, dass ein wahrer Gefahrenhinweis missachtet wird, reduziert werden kann (Kalisch et al. 2006b, S. 9503). Insgesamt wurden neun Studien zum Thema „Furchtextinktion“ in diese Metaanalye inkludiert. 
Sechs der neun verwendeten Studien über die Furchtextinktion benutzten als unkonditionierten Stimulus Schmerz in Form eines ungefährlichen Stromstoßes, eine weitere nutzte als Reiz eine schmerzhafte Dehnung des Ösophagus und eine weitere Studie verwendete als Schmerzreiz einen 1000-Hz-Ton. Die Angstlöschung erfolgte durch Ausbleiben des Schmerzreizes. Eine letzte bildgebende Studie zeigte Probanden Bilder, die einen Gewinn oder Verlust von Punkten bedeuteten und löschte die Angst durch veränderte Ergebnisse, die in Kombination mit den Bildern präsentiert wurden.

In allen relevanten Studien wurden die Koordinaten zum Thema „Furchtextinktion“ extrahiert. In einer Studie wurde darauf verwiesen, dass sich die aktivierten Hirnareale auf einen frühen Zeitpunkt im Prozess der Furchtextinktion beziehen (Early Extinction) (Spoormaker et al. 2010), in einer weiteren Studie bezogen sich die relevanten Koordinaten auf einen späten Zeitpunkt beim Erlernen der Furchtextinktion (Late Extinction Learning) (Milad et al. 2007). Des Weiteren wurden aus zwei Studien Gehirnkoordinaten entnommen, die sich auf die Wiedergabe des Löschgedächtnisses beziehen (Recall of Fear Extinction) (Milad et al. 2007, Spoormaker et al. 2010). In den restlichen Studien wurden keine Verweise auf Besonderheiten bezüglich der extrahierten Koordinaten gemacht.

\subsection{Placebostudien}

Es wurden 13 Studien in die Metaanalyse miteinbezogen, die sich mit dem Placeboeffekt beschäftigten. Der Placebogebrauch ist eine weitere Möglichkeit, negative Emotionen zu reduzieren.

In 10 der bildgebenden Studien wurden den Probanden Schmerzreize unterschiedlicher Intensitäten zugefügt (fünf Hitzereize, zwei Laser-Stimulationen, ein Radiotracer, eine Dehnung des Ösophagus, ein elektrischer Schock). Anstatt eines Schmerzmittels oder schmerzlindernder Akupunktur wurde den Probanden zuvor ein Placebo verabreicht mit dem Hinweis, die Substanz sei ein schmerzlinderndes Medikament.

In fünf Studien wurden als Placebos unechte analgetische Cremes (meist Basis Hautcreme), in zwei Studien vorgetäuschte Akupunkturnadeln und in drei Studien Injektionen mit einer Kochsalzlösung verwendet. Das Schmerzempfinden der Probanden wurde getestet und die Stärke des Schmerzes 
von den Versuchsprobanden bewertet. Der Schmerzreiz wurde durch die Placeboeinnahme als weniger schmerzhaft empfunden.

Die Reaktionen der Probanden zur schmerzhaften Stimulierung wurde zum einen unter der Placeboeinnahme und zum anderen unter Bedingungen ohne Placebobehandlung als Kontrollversuch mit Hilfe von ereignisgebundenem fMRI beurteilt. Durch diesen direkten Vergleich war es möglich, Gehirnregionen zu identifizieren, die während der Schmerzreduktion und somit der Reduktion negativer Emotionen aktiviert waren.

Das Studiendesign der untersuchten Placebostudien zeigte sich wie folgt:

Unter den Studien über die Placebobehandlung war die Bedingung der Schmerzzuführung bei den Probanden nicht zwingend. Eine irreführende Erwartung, etwas weniger Negatives zu erfahren zählte auch zu diesem Studientyp (Diekhof et al. 2011a). Die Studien zeigten, dass die Erwartungsmanipulation durch die Placebos die Wahrnehmung von aversiven Ereignissen und die Begleitgehirnaktivierung änderten (Sarinopoulos et al. 2006). Zu dieser Gruppe zählten drei Studien (Diekhof et al. 2011b, Petrovic et al. 2005, Sarinopoulos et al. 2006)

In zwei weiteren Studien wurden den Probanden Angst erzeugende Bilder gezeigt und ihnen ein Placebo (Kochsalzlösung) anstelle einer Injektion mit einer angstlindernden Substanz (Benzodiazepam) verabreicht. Die Probanden sollten die Aversivität der Bilder bewerten in dem Bewusstsein, ein Benzodiazepam bekommen zu haben (Petrovic et al. 2005).

Diekhof et al. (2011b) zeigten ihren Probanden Bilder mit angsterfüllten Gesichtern und veränderten die Wahrnehmung der Versuchsteilnehmer ,positiv“ durch vorherige mentale Vorstellungsstrategien über weniger negative Gesichter.

Sarinopoulos et al. (2006) präsentierten in ihrer Studie den Probanden bittere Geschmackslösungen und veränderten zuvor die Erwartungen der Versuchsteilnehmer durch Hinweise, die die zu verabreichenden Geschmacksrichtungen als weniger aversiv beschrieben.

In einigen Placebostudien wurden die Erwartungen der Probanden manipuliert („,This medication is thought to have analgesic effects through the activation of natural brain systems that suppress pain“ Scott et al. 2008, S. 222) um die Wahrnehmung der aversiven Reize zum „Positiven“ zu verändern (Scott et al. 2008, Wager et al. 2004, Sarinopoulos et al. 2006, Diekhof et al. 2011b). 


\subsection{Emotionsregulationsstudien}

In 20 der 23 bildgebenden Emotionsregulationsstudien wurden die Probenden aversiven Reizen ausgesetzt, indem ihnen abstoßende, unerfreuliche Bilder und Filme präsentiert wurden. Die Probanden sollten ihre negativen Gefühle vermindern, indem sie die präsentierte Situation mit Abstand betrachteten und eine emotionale Distanz dazu aufbauten. Auch die Betrachtung aus der Perspektive einer dritten Person eröffnete die Möglichkeit, dieses Ziel zu erreichen. Die Versuchsteilnehmer sollten sich vorstellen, mit dem präsentierten Kontext und den Individuen nicht persönlich verbunden zu sein, und wurden darum gebeten, sich eine positive Entwicklung des dargebotenen Ereignisses zu denken und es somit neu zu interpretieren (Ochsner et al. 2004). Man versuchte nicht, die negativen Emotionen und die Angst der Probanden durch Ablenkung, wie beispielsweise das Lösen einer geistig anspruchsvollen Aufgabe (Kellermann et al. 2011), zu reduzieren, da Formen von Ablenkung die Gefühlsverarbeitung zerstören (Ochsner et al. 2004). Ablenkung produziert Inhalte im Arbeitsgedächtnis, die nicht in Beziehung zu dem zu unterdrückenden angstverbundenen Material stehen, im Gegensatz zu dem Prozess der willentlichen Neubewertung, bei dem Inhalte im Arbeitsgedächtnis geschaffen werden durch die Neuinterpretierung von angstverbundenem Material, das, obwohl es ,sicher“ ist, trotzdem mit der Angst in Zusammenhang steht (Kalisch et al. 2006c). Die Versuchsteilnehmer wurden daher dazu aufgefordert, die präsentierte Situation neu zum Positiven zu bewerten (Ochsner et al. 2004).

In einer weiteren Studie war es die Aufgabe der Probanden, sich auf negative autobiographische Erinnerungen zu konzentrieren und die Rumination der negativen Gefühle zu minimieren (Kross et al. 2009). Die Teilnehmer sollten die erlebten Ereignisse akzeptieren, aber versuchen sich von ihnen zu distanzieren, um die Angst zu regulieren.

In den übrigen zwei Studien wurde den Probanden ein Schmerzreiz in Form eines leichten elektrischen Stromstoßes zugefügt. Die Aufgabe der Teilnehmer war es, die Situation neu zu bewerten (Reappraisal) und zu versuchen, sich - beispielsweise - etwas in der Natur vorzustellen, um sich zu beruhigen und die negativen Emotionen zu reduzieren (Delgado et al. 2008).

Studien, in denen die Probanden ihre physiologischen negativen Emotionen und ihre Angstreaktion einfach unterdrückten (Suppression), wurden ausgeschlossen. Dies wurde getan, weil Unterdrückung normalerweise zu geringfügigen oder keinen Änderungen in der Erfahrung des 
emotionalen Ereignisses (Gross 2002) führt und auf diese Art wahrgenommene Angst im Vergleich mit anderen kognitiven Strategien weniger wirksam reduziert wird (Goldin et al. 2007).

In allen Studien wurden zum Vergleich Versuche durchgeführt, in denen die Probanden ihren Gefühlen freien Lauf lassen sollten, um direkte Vergleiche bezüglich der aktivierten Gehirnregionen ziehen zu können.

Interessant war bei den Emotionsregulationsstudien die Betrachtung der Geschlechterverteilung, denn die Tatsache, dass Frauen emotionaler als Männer sind, ist ein allgemeines Klischee (Mc Rae et al. 2008). 10 Studien untersuchten nur weibliche Probanden (Drabant et al. 2008, Eippert et al. 2007, Goldin et al. 2007, Harenski und Hamann 2006, Kim und Hamann 2007, Mak et al. 2009, McRae et al. 2009, Ochsner et al. 2002, Ochsner et al. 2004, Walter et al. 2009). Es wurde deshalb eine weitere Metaanalyse durchgeführt, mit Studien, die weibliche und auch männliche Probanden anteilig mit einer ähnlichen Gewichtung untersuchten (siehe Tabelle 3.4). Dies war wichtig, um mögliche Fehler der Ergebnisse durch den starken Anteil an weiblichen Probanden aufzudecken.

\subsection{Koordinatenselektion}

Es wurden zum einen nur die Koordinaten mit verstärkter Aktivierung und zum zweiten die Koordinaten mit verringerter Aktivität bei der Furchtextinktion, der Emotionsregulation und der Placebobehandlung gewählt.

Bei den Furchtextinktionsstudien wurden die aktivierten Gehirnkoordinaten, bei denen dem CS kein UCS (wie z.b. ein Schock) mehr folgte (,CS+“") im Vergleich zur Situation, bei der dem CS ein UCS folgte (,CS-“) extrahiert (siehe Tabelle 2.1). Weiterhin wurden auch die Koordinaten aus den Studien in die Metaanalyse miteinbezogen, die den Prozess der Furchtextinktion in unterschiedliche Phasen einteilten (beispielsweise bei Spoormaker et al. 2010 Frühes Löschen und Wiederabruf des Löschgedächtnisses (,Recall“)) (Diekhof et al. 2011a).

Bei den Placebostudien wurden die Koordinaten mit Aktivierungen bei der Placeboeinnahme extrahiert, die im direkten Vergleich zu den neuronalen Aktivierungen bei der Wahrnehmung eines Schmerzreizes ohne Placeboeinnahme standen (siehe Tabelle 2.2). Bei der Kontrolluntersuchung ohne Placebobehandlung bekamen die Probanden vor der Reizdarbietung den Hinweis, eine Creme 
ohne analgetischen Effekt zu erhalten (z.B. Wager et al. 2004 (1)) oder keine Akupunkturbehandlung und somit keinen analgetischen Effekt zu erfahren (z.B. Kong et al. 2009). Aus den relevanten Emotionsregulationsstudien wurden die Koordinaten extrahiert, die von einer verstärkten Gehirnaktivierung während der Angstreduktion durch verschiedene Formen der Angstregulation bei der Wahrnehmung oder der Erwartung eines negativen Ereignisses (beispielsweise Schmerzreiz oder unerfreuliche Bildern) berichteten und im Vergleich zu den Gehirnkoordinaten standen, die bei der Wahrnehmung der negativen Emotionen und der Angst Aktivierungen zeigten (siehe Tabelle 2.3 bzgl. der relevanten Kontraste in den einzelnen Studien). Wenn dieser Kontrast in den ausgewählten Studien nicht untersucht wurde, wurden die Koordinaten aus dem Vergleich - Hirnaktivität bei Angstverringerung zur Hirnaktivität emotionaler Neutralität - für die Metaanalyse herangezogen. In Studien, in denen beide Vergleiche behandelt wurden, wurden die Koordinaten aus dem stärkeren Kontrast bevorzugt extrahiert (z.B. Angstreduktion gegen Angstwahrnehmung). Bei Verweisen auf weitere verstärkte Gehirnaktivierungen, die mit der Angstreduktion einhergingen, wurden diese Koordinaten auch der Metaanalyse zugeführt (z.B. Wager et al. 2008, S.1041 „Correlations between Reappraisal Aktivation and Reappraisal Success in reported Experience“").

Die Studien, die von separaten Ergebnissen für männliche und weibliche Probanden berichteten, wurden als gemeinsame Daten angesehen (Hinweise in den Ergebnistabellen 2.1-2.3 ersichtlich). Die Koordinaten wurden vorrangig aus Tabellen der verwendeten Studien entnommen. Sofern keine Tabellen vorlagen, wurden Abbildungslegenden sowie der Text nach Koordinaten durchsucht. Die Koordinaten außerhalb der Maske (Foki outside the area) wurden gestrichen, da sie möglicherweise fremde Aktivierungen an den Rand ihrer Maske bringen, und somit kein Zentrum der Masse abzeichnen (Laird 2009). Redundante Koordinaten wurden entfernt. Zusätzlich wurde die Anzahl der Probanden mit in die Berechnungen einbezogen.

\subsection{Die Metaanalyse}

Die Metaanalyse eröffnet die Möglichkeit, quantitative Ergebnisse verschiedener, aber ähnlicher Studien zusammenzufassen. Einzeln betrachtet können Untersuchungen zu kleine Fallzahlen aufweisen oder in ihrem Rahmen limitiert sein, um generelle Schlüsse zu ziehen. Durch eine 
Metaanalyse kann die Tendenz einzelner Studien bekräftigt werden (DerSimonian und Laird 1986).

Die strukturbasierte Metaanalyse konzentriert sich auf spezifische anatomische Regionen und versucht globale Koaktivierungen in den Regionen festzustellen, während die Probanden verschiedene Aufgaben in unterschiedlichen Versuchen lösen.

Im Gegensatz dazu steht die funktionsbasierte Metaanalyse, die eine Sammlung von ähnlichen Studien mit gleichen experimentellen Entwürfen untersucht. Die Theorie zur strukturbasierten Metaanalyse geht davon aus, dass Koordinatengruppen, die bei verschiedenen Versuchen aktiviert sind, funktionell verbundene Netze im Gehirn identifizieren können. Die strukturbasierte Metaanalyse erhöht die Möglichkeit, Ergebnisse zu generalisieren (Laird et al. 2009).

\subsection{Activation likelihood estimation (ALE)}

Nach der Literaturrecherche erfolgte die Activation-likehood-estimation (ALE)-Metaanalyse. Durchgeführt wurde sie mit der Software GingerALE in der Version 2.0 (Laird et al. 2005b, Turkeltaub et al. 2002).

„ALE ist seit ihrer Einführung (Chein et al. 2002, Turkeltaub et al. 2002) eine anerkannte Methode mit einer hohen Spezifität und Sensibilität und wird bei vielen Untersuchungen normaler Gehirnfunktionen (Costafreda et al. 2008, Decety und Lamm 2007, Eickhoff et al. 2009, Grosbras et al. 2005, Sörös et al. 2009, Spreng et al. 2009), in Studien von neuropsychologischen und neurologischen Störungen, wie Schizophrenie (Glahn et al. 2005, Minzenberg et al. 2009, Ragland et al. 2009), zwanghaften Störungen (Menzies et al. 2008), Depressionen (Fitzgerald et al. 2008), und Entwicklungsstottern (Brown S et al. 2005) angewandt“ (Laird et al. 2009, S.3).

Auf der Internetseite brainmap.org ist das Herunterladen der neuesten GingerALE-Version möglich. BrainMap wurde dafür entwickelt, die quantitative Metaanalyse von Ergebnissen aus sogenannten „neuroimaging“-Studien zu erleichtern und unterstützt die Methode, Aktivierungswahrscheinlichkeiten einzuschätzen (ALE). BrainMAP ist eine Datenbank, die es ermöglicht, Verhaltensfunktionen mit Gehirnregionen in Verbindung $\mathrm{zu}$ bringen durch Visualisierung der Koordinaten und ihre zugehörigen Metadaten, um so Ergebnisse von Studien zu vergleichen (Laird et al. 2009). 
Die Berechnungen mit GingerALE wurden mit allen drei Studientypen (Furchtextinktion, Emotionsregulation, Placeboeffekt) durchgeführt, um sie anschließend anhand der Ergebnisse untereinander zu vergleichen.

In ALE sind 3 D Koordinaten (x, y, z) im stereotaktischen Raum von einer Sammlung von Studien vereinigt (Laird et al. 2005b, S.156). Dieser voxelbasierte Ansatz ermöglicht die Analyse der räumlichen Verteilung und Konkordanz von Gehirnkoordinaten aus den unterschiedlichen Studien. Das Ergebnis der ALE-Metaanalyse ist eine statistische Landkarte des ganzen Gehirns. Das Zentrum eines Aktivitätsclusters entspricht dem Zentrum einer GausWahrscheinlichkeitsverteilung, wobei das Zentrum des Clusters die höchste Signifikanz und somit den höchsten ALE-Wert besitzt. Es wurde die Wahrscheinlichkeit der Aktivierung für jeden Voxel für jede der drei Aufgaben (Furchtextinktion, Emotionsregulation, Placebobehandlung) geschätzt, wobei bei jeder Aufgabe je der vollständige Satz der relevanten Studien betrachtet wurde (Laird et al. 2005b). Die ALE-Statistik ist auf jeden Voxel im Gehirn berechnet (Laird et al. 2005a, S.7). Jede Studie präsentiert eine unterschiedliche Anzahl an Fokussen. Da alle Fokusse gleichermaßen bei der ALE-Methode bewertet werden, haben die Studien, die von einer größeren Anzahl an Fokussen berichten, einen stärkeren Einfluss (Laird et al. 2005b, S. 156).

In dieser Phase der Metaanalyse wurden die Talairach-Koordinaten und die MNI- Koordinaten aus den relevanten Studien extrahiert. Die Talairach-Koordinaten geben eine Richtlinie zur Lage der Achsen und zur Standardisierung der Größe individuell unterschiedlicher Gehirne (Talairach und Szikla 1967, Talairach und Tournoux 1988). Weiterhin gibt es eine vom Talairach-Gehirn abweichende Empfehlung vom neurologischen Institut Montreal (MNI). Studien, die von Talairach-Koordinaten berichteten, wandelte ich mit Hilfe der Lancaster-Transformation (icbm2tal) bei GingerALE (Lancaster et al. 2007) in die MNI-Form um. Ich wählte die Koordinaten in MNI-Form, weil mehr Studien von MNI-Koordinaten anstatt von Talairach-Koordinaten berichteten. Jede Transformation kann zu Fehlern führen, die möglichst gering gehalten werden sollten.

\subsection{Durchführung der ALE-Metaanalyse}

ALE-Landkarten wurden entsprechend dem von Turkeltaub et al. (2002) beschriebenen Verfahren geschaffen. 
Die Berechnungen bei GingerALE erfolgten in drei Schritten.

Der erste Schritt berechnet die ALE-Werte für jeden Voxel im Gehirn und führt einen Test durch, um die Nullverteilung bei jedem Voxel zu bestimmen. Die Halbwertsbreite (Breite der Gaußfunktion bei halber Amplitude= FWHM-Wert= Full Width at Half Maximum) wurde empirisch bestimmt (siehe Eickhoff et al. 2009 für Einzelheiten).

Ginger ALE erzielte in diesem ersten Schritt zwei Ergebnisse: Zum einen die ALE-Werte (Extrema Value) der Fokusse entsprechend und zum anderen die P-Werte (Überschreitungswahrscheinlichkeit, Signifikanzwert) von jedem Voxel (Laird 2009).

Im folgenden Schritt wurde der Grenzwert (Threshold) der ALE-Karte erstellt mit Hilfe der PWerte aus dem ersten Schritt und mit dem Algorithmus von Thomas E. Nichols ${ }^{1}$. Es wurde das Signifikanzniveau auf 0.05 festgelegt sowie eine minimale Clustergröße von $100 \mathrm{~mm} \wedge 3$ gewählt. Nur Voxel, die als statistisch signifikant eingestuft wurden, bekamen einen ALE-Wert zugewiesen. In diesem Schritt wurde die Thresholded-Landkarte erstellt (Laird 2009).

Im letzten Schritt wurde die Clusteranalyse der Thresholded-Landkarte durchgeführt.

\subsection{Konkretes Vorgehen bei Durchführung der Metaanalyse}

Es wurden separate ALE-Landkarten für Emotionsregulation, Furchtextinktion und Placebobehandlung berechnet, um regionale Konkordanz in Aktivierungsmustern innerhalb der Gruppen zu beurteilen. Insgesamt wurden ALE-Landkarten für die Verteilung der Aktivierungen während der Regulation negativer Emotionen jeweils für die Furchtextinktionsstudien, die Placebostudien und die Emotionsregulationsstudien berechnet. Zusätzlich wurden Berechnungen für die Verteilung der Aktivierungen der Gehirnregionen für alle drei Studientypen zusammen durchgeführt, um Überlappungszentren herauszuarbeiten. Weiterhin erfolgte eine Berechnung speziell für Aktivierungen in willentlichen Emotionsregulationsstudien, die Probanden beiderlei Geschlechts miteinbezogen, da ein Großteil der Emotionsregulationsstudien der allgemeinen Metaanalyse nur mit Frauen durchgeführt wurden und diese möglicherweise stärkere Emotionen zeigen, die folglich eine stärkere emotionale Regulation erfordern. Dazu wurde

\footnotetext{
${ }^{1}$ Verfügbar im Internet: http://www.sph.umich.edu/ nichols/FDR/
} 
die Paarmetaanalyse durchgeführt, in der ,Placeboeffekt und willentliche Emotionsregulation' zusammen untersucht wurden, um mögliche Übereinstimmung von Gehirnarealen mit verstärkter Aktivierung während der Reduktion negativer Emotionen bei geistig anspruchsvolleren Gefühlsregulationsstrategien aufzudecken. Als weitere Gemeinsamkeit hatten die beiden Studientypen die permanente Darbietung des negativen Reizes, im Gegensatz zur Furchtextinktion. Abschließend erfolgten noch die Berechnungen der Verteilung von verminderter Gehirnaktivität während der Regulation negativer Emotionen jeweils für alle drei Studientypen einzeln und schließlich zusammen.

Insgesamt wurden neun bildgebende Studien mit 53 Fokussen zum Thema Furchtextinktion, 13 Placebostudien mit 128 Fokussen und 23 Studien über Emotionsregulation mit 196 Fokussen gefunden.

Mit Hilfe der Mango Software 2.3.2 ${ }^{2}$ wurden die erzeugten Gehirnkarten auf ein MNIStandardgehirn (Colin T1-template ${ }^{3}$ ) visualisiert.

\footnotetext{
${ }^{2}$ Verfügbar im Internet: http://ric.uthscsa.edu/mango

${ }^{3}$ Verfügbar im Internet: http:// brainmap.org/ale/index.html.
} 
Tabelle 2.1: Furchtextinktionsstudien

\begin{tabular}{|c|c|c|c|c|c|c|}
\hline Studie & $\begin{array}{c}\text { Anzahl } \\
\text { der } \\
\text { Proban- } \\
\text { den }\end{array}$ & $\begin{array}{l}\text { Geschlechter- } \\
\text { verteilung } \\
(\mathbf{f} / \mathbf{m})^{*}\end{array}$ & Art der Stimulation & Kontrast & $\begin{array}{l}\text { Anzahl } \\
\text { Fokusse }\end{array}$ & Modalität \\
\hline $\begin{array}{l}\text { Finger et al. } \\
\quad 2008\end{array}$ & 20 & $10 / 10$ & $\begin{array}{c}\text { Bilder gepaart mit } \\
\text { Verlustsituation }\end{array}$ & $\begin{array}{c}\text { Furchtextinktion> } \\
\text { Kontrolle } \\
\text { (frühe Extinktion) }\end{array}$ & 2 & $\begin{array}{l}\text { Visuelle } \\
\text { Wahrneh- } \\
\text { mung }\end{array}$ \\
\hline $\begin{array}{c}\text { Kalisch et al. } \\
\text { 2006b }\end{array}$ & 17 & $8 / 9$ & $\begin{array}{l}\text { Gesichter gepaart mit } \\
\text { elektrischen Schocks }\end{array}$ & $\begin{array}{c}\text { (CS+> CS-) ext> } \\
(\mathrm{CS}+>\mathrm{CS}-) \mathrm{u} \\
\text { (Abruf (recall) } \\
\text { Löschgedächtnis) }\end{array}$ & 11 & Schmerz \\
\hline $\begin{array}{l}\text { Knight et al. } \\
2004\end{array}$ & 10 & Keine Angabe & $\begin{array}{l}\text { Lichter gepaart mit } \\
\text { elektrischen Schocks }\end{array}$ & $\begin{array}{c}\text { CS+ext>CS+u } \\
\text { (frühe Extinktion) }\end{array}$ & 1 & Schmerz \\
\hline $\begin{array}{l}\text { Lang et al. } \\
\qquad 2009\end{array}$ & 21 & $7 / 14$ & $\begin{array}{c}\text { Farbiger } \\
\text { Hintergrund gepaart } \\
\text { mit elektrischem } \\
\text { Schock }\end{array}$ & $\begin{array}{c}\text { CS+ ext>CS- } \\
\text { (frühe Extinktion) } \\
\text { Frühe Extinktion > } \\
\text { Späte Aneignung }\end{array}$ & 6 & Schmerz \\
\hline $\begin{array}{l}\text { Milad et al. } \\
\qquad 2007\end{array}$ & 14 & $7 / 7$ & $\begin{array}{l}\text { Lichter gepaart mit } \\
\text { elektrischen Schocks }\end{array}$ & $\begin{array}{c}\text { CS+ ext> CS- } \\
\text { (späte Extinktion) } \\
\text { CS+ ext> CS+u (Abruf } \\
\text { Löschgedächtnis) }\end{array}$ & 16 & Schmerz \\
\hline $\begin{array}{l}\text { Schiller et al. } \\
2008\end{array}$ & 17 & $8 / 9$ & $\begin{array}{l}\text { Gesichter gepaart mit } \\
\text { elektrischen Schocks }\end{array}$ & $\begin{array}{c}\text { Neu CS-> neu CS+ } \\
\text { (späte Umkehr) }\end{array}$ & 1 & Schmerz \\
\hline $\begin{array}{c}\text { Soliman et al. } \\
2010\end{array}$ & 35 & $15 / 20$ & $\begin{array}{l}\text { Geometrische } \\
\text { Formen } \\
\text { gepaart mit } \\
\text { 1000-Hz-Ton }\end{array}$ & $\begin{array}{c}\text { CS+ ext (frühe } \\
\text { Extinktion bei } \\
\text { Val/Val Trägern) }\end{array}$ & 1 & $\begin{array}{c}\text { Auditorische } \\
\text { Wahrneh- } \\
\text { mung }\end{array}$ \\
\hline $\begin{array}{c}\text { Spoormaker } \\
\text { et al. } 2010\end{array}$ & 16 & $0 / 16$ & $\begin{array}{l}\text { Geometrische } \\
\text { Formen gepaart mit } \\
\text { elektrischen Schocks }\end{array}$ & $\begin{array}{c}\text { CS+ ext > CS- } \\
\text { (frühe Extinkton) } \\
\text { CS+ ext > CS+ u (Abruf } \\
\text { Löschgedächtnis) }\end{array}$ & 7 & Schmerz \\
\hline $\begin{array}{l}\text { Yágüez et } \\
\text { al.2005 }\end{array}$ & 8 & $3 / 5$ & $\begin{array}{c}\text { Geometrische } \\
\text { Formen gepaart mit } \\
\text { schmerzhafter } \\
\text { ösophagealer } \\
\text { Distension }\end{array}$ & $\begin{array}{c}\text { CS+ ext>CS- } \\
\text { (frühe Extinktion) }\end{array}$ & 8 & Schmerz \\
\hline
\end{tabular}

*Diese Studien beinhalten 58 weibliche und 90 männliche Probanden. 
Tabelle 2. 2: Placebostudien

\begin{tabular}{|c|c|c|c|c|c|c|}
\hline Studie & $\begin{array}{l}\text { Anzahl } \\
\text { der } \\
\text { Proban- } \\
\text { den }\end{array}$ & $\begin{array}{c}\text { Geschlechter- } \\
\text { verteilung } \\
(\mathbf{f} / \mathbf{m})^{*}\end{array}$ & Art der Stimulation & Kontrast & $\begin{array}{l}\text { Anzahl } \\
\text { Fokusse }\end{array}$ & Modalität \\
\hline Bingel et al. 2006 & 19 & $4 / 15$ & $\begin{array}{c}\text { Schmerzhafte Laser } \\
\text { Stimulation gepaart } \\
\text { mit analgetischer } \\
\text { Placebocreme }\end{array}$ & $\begin{array}{c}\text { Placebo> Kein } \\
\text { Placebo }\end{array}$ & 2 & Schmerz \\
\hline $\begin{array}{c}\text { Diekhof et al. } \\
\text { 2011b }\end{array}$ & 9 & $4 / 5$ & $\begin{array}{l}\text { Angstinduzierende } \\
\text { Gesichter gepaart mit } \\
\text { irreführendem } \\
\text { vorwegnehmendem } \\
\text { Hinweis und Bild }\end{array}$ & $\begin{array}{l}\text { Sinnestäuschung> } \\
\text { Echte Wahrnehmung }\end{array}$ & 9 & $\begin{array}{c}\text { Visuelle } \\
\text { Wahrneh- } \\
\text { mung }\end{array}$ \\
\hline $\begin{array}{c}\text { Eippert et al. } \\
2009\end{array}$ & 20 & $0 / 20$ & $\begin{array}{c}\text { Hitzereiz gepaart mit } \\
\text { analgetischer } \\
\text { Placebocreme }\end{array}$ & 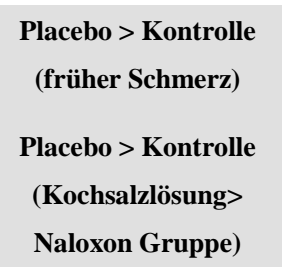 & 8 & Schmerz \\
\hline Kong et al. 2006 & 24 & $11 / 13$ & $\begin{array}{l}\text { Hitzereiz gepaart mit } \\
\text { analgetischer } \\
\text { Akupunktur- } \\
\text { täuschung }\end{array}$ & $\begin{array}{c}\text { Placebo > Kontrolle } \\
\text { Korrelation der } \\
\text { Aktivierung bei } \\
\text { Placeboanalgesie }\end{array}$ & 7 & Schmerz \\
\hline Kong et al. 2009 & 12 & $6 / 6$ & $\begin{array}{l}\text { Hitzereiz gepaart mit } \\
\text { analgetischer } \\
\text { Akupunktur- } \\
\text { täuschung }\end{array}$ & $\begin{array}{c}\text { Hohe Erwartung } \\
\text { Placeboeffekt > } \\
\text { Kontrolle }\end{array}$ & 3 & Schmerz \\
\hline $\begin{array}{l}\text { Petrovic et al. } \\
2002\end{array}$ & 9 & $0 / 9$ & $\begin{array}{c}\text { Hitzereiz gepaart mit } \\
\text { einer analgetischen } \\
\text { Placeboinjection }\end{array}$ & Placebo $>$ Schmerz & 2 & Schmerz \\
\hline $\begin{array}{c}\text { Petrovic et al. } \\
2005 \text { (2) }\end{array}$ & 11 & $11 / 0$ & $\begin{array}{l}\text { Angstinduzierende } \\
\text { Gesichter gepaart mit } \\
\text { angstlösender } \\
\text { Placeboinjection }\end{array}$ & $\begin{array}{c}\text { Placebo > Kontrolle } \\
\text { Placebo> Kontrolle } \\
\text { (unerfreulich > } \\
\text { neutrale Bilder) } \\
\text { Korrelation mit } \\
\text { Placeboeffekt }\end{array}$ & 24 & $\begin{array}{c}\text { Visuelle } \\
\text { Wahrneh- } \\
\text { mung }\end{array}$ \\
\hline
\end{tabular}


Tabelle 2.2 Placebostudien (Fortsetzung)

\begin{tabular}{|c|c|c|c|c|c|c|}
\hline Studie & $\begin{array}{l}\text { Anzahl } \\
\text { der } \\
\text { Proban- } \\
\text { den }\end{array}$ & $\begin{array}{c}\text { Geschlechter- } \\
\text { verteilung } \\
(\mathbf{f} / \mathbf{m})^{*}\end{array}$ & Art der Stimulation & Kontrast & $\begin{array}{c}\text { Anzahl } \\
\text { Fokusse }\end{array}$ & Modalität \\
\hline $\begin{array}{c}\text { Sarinopoulos et } \\
\text { al. } 2006\end{array}$ & 43 & $19 / 24$ & $\begin{array}{c}\text { Negativer } \\
\text { Geschmacksreiz } \\
\text { gepaart mit einem } \\
\text { vorwegnehmenden, } \\
\text { positiveren, } \\
\text { irreführenden } \\
\text { Hinweis }\end{array}$ & $\begin{array}{c}\text { Korrelation von } \\
\text { vorwegnehmender } \\
\text { Aktivierung während } \\
\text { irreführender } \\
\text { Erwartung mit } \\
\text { Amygdalareaktion } \\
\text { während } \\
\text { Sinnestäuschung }\end{array}$ & 7 & Geschmack \\
\hline $\begin{array}{c}\text { Scott et al. } \\
2008 * *\end{array}$ & 20 & $11 / 9$ & $\begin{array}{c}\text { Infusion einer } \\
\text { Salzlösung in den M. } \\
\text { Masseter gepaart mit } \\
\text { Placeboinjektion }\end{array}$ & Placebo & 15 & Schmerz \\
\hline $\begin{array}{l}\text { Wager et al. } 2004 \\
\text { (1) }\end{array}$ & 24 & Keine Angabe & $\begin{array}{c}\text { Elektrischer Schock } \\
\text { gepaart mit } \\
\text { analgetischer } \\
\text { Placebocreme }\end{array}$ & $\begin{array}{c}\text { Korrelation von } \\
\text { vorwegnehmender } \\
\text { Aktivierung } \\
\text { (Placebo > Kontrolle) } \\
\text { mit berichteter } \\
\text { Placebowirkung }\end{array}$ & 14 & Schmerz \\
\hline $\begin{array}{c}\text { Wager et al. } 2004 \\
\text { (2) }\end{array}$ & 23 & Keine Angabe & $\begin{array}{c}\text { Schmerzhafter } \\
\text { Hitzereiz gepaart mit } \\
\text { analgetischer } \\
\text { Placebocreme }\end{array}$ & $\begin{array}{c}\text { Placebo > Kontrolle } \\
\text { (early anticipation) } \\
\text { Placebo > Kontrolle } \\
\text { (late anticipation) }\end{array}$ & 8 & Schmerz \\
\hline $\begin{array}{l}\text { Watson et al. } \\
2009\end{array}$ & 11 & $6 / 5$ & $\begin{array}{c}\text { Schmerzhafter } \\
\text { Laserreiz gepaart mit } \\
\text { analgetischer } \\
\text { Placebocreme }\end{array}$ & $\begin{array}{c}\text { Placebo > Kontrolle } \\
\text { (postconditioning > } \\
\text { preconditioning) }\end{array}$ & 6 & Schmerz \\
\hline $\begin{array}{c}\text { Zubieta et al. } \\
2005 * * *\end{array}$ & 14 & $0 / 14$ & $\begin{array}{c}\text { Infusion einer } \\
\text { Salzlösung in den M. } \\
\text { Masseter gepaart mit } \\
\text { Placeboinjektion }\end{array}$ & Placebo & 5 & Schmerz \\
\hline
\end{tabular}

* Diese Studien beinhalten 72 weibliche und 120 männliche Probanden.

** Diese PET-Studie testete die placeboinduzierten neuronalen Veränderungen in den $\mu$-opioiden und dopaminergen Systemen.

*** Diese PET-Studie testete die placeboinduzierten neuronalen Veränderungen in dem $\mu$-opioiden System. 
Tabelle 2.3: Emotionsregulationsstudien

\begin{tabular}{|c|c|c|c|c|c|c|}
\hline Studie & $\begin{array}{l}\text { Anzahl der } \\
\text { Probanden }\end{array}$ & $\begin{array}{c}\text { Geschlechter- } \\
\text { verteilung } \\
(\mathbf{f} / \mathbf{m})^{*}\end{array}$ & $\begin{array}{c}\text { Art der } \\
\text { Stimulation }\end{array}$ & Kontrast & $\begin{array}{c}\text { Anzahl } \\
\text { Fokusse }\end{array}$ & Modalität \\
\hline $\begin{array}{c}\text { Banks et al. } \\
\quad 2007\end{array}$ & 14 & $8 / 6$ & $\begin{array}{c}\text { negative IAPS- } \\
\text { Bilder und } \\
\text { emotionale } \\
\text { Regulation durch } \\
\text { Neuinterpretation }\end{array}$ & $\begin{array}{c}\text { Neubewerten } \\
(\text { reappraise) }> \\
\text { Beibehalten } \\
\text { (maintain) } \\
\text { (Zusammenhang mit } \\
\text { Amygdala) }\end{array}$ & 9 & $\begin{array}{c}\text { Visuelle } \\
\text { Wahrnehmung }\end{array}$ \\
\hline $\begin{array}{l}\text { Delgado et } \\
\text { al. } 2008\end{array}$ & 12 & $6 / 6$ & $\begin{array}{c}\text { Elektrischer } \\
\text { Schock und } \\
\text { emotionale } \\
\text { Regulation durch } \\
\text { vorausschauende } \\
\text { beruhigende Bilder }\end{array}$ & $\begin{array}{l}\text { Regulation > } \\
\text { Beibehalten }\end{array}$ & 6 & Schmerz \\
\hline $\begin{array}{c}\text { Domes et al. } \\
2010\end{array}$ & 33 & $17 / 16$ & $\begin{array}{c}\text { negative IAPS- } \\
\text { Bilder und } \\
\text { emotionale } \\
\text { Regulation durch } \\
\text { Distanzierung }\end{array}$ & $\begin{array}{c}\text { Verminderung > } \\
\text { Beibehalten }\end{array}$ & 16 & $\begin{array}{c}\text { Visuelle } \\
\text { Wahrnehmung }\end{array}$ \\
\hline $\begin{array}{c}\text { Drabant et } \\
\text { al. } 2008\end{array}$ & 56 & $56 / 0$ & $\begin{array}{c}\text { ärgerliche und } \\
\text { angsterzeugende } \\
\text { Gesichter und } \\
\text { emotionale } \\
\text { Regulation durch } \\
\text { gewöhnliche } \\
\text { Neubewertungs- } \\
\text { strategie }\end{array}$ & $\begin{array}{c}\text { Zusammenhang von } \\
\text { gewöhnlicher } \\
\text { Neubeurteilungs- } \\
\text { strategie mit } \\
\text { eingeschlossener } \\
\text { Verarbeitung } \\
\text { emotionaler } \\
\text { Gesichter }\end{array}$ & 3 & $\begin{array}{c}\text { Visuelle } \\
\text { Wahrnehmung }\end{array}$ \\
\hline $\begin{array}{c}\text { Eippert et } \\
\text { al. } 2007\end{array}$ & 24 & $24 / 0$ & $\begin{array}{c}\text { negative IAPS- } \\
\text { Bilder und } \\
\text { emotionale } \\
\text { Regulation durch } \\
\text { Distanzierung }\end{array}$ & $\begin{array}{c}\text { Verminderung > } \\
\text { Anschauen }\end{array}$ & & $\begin{array}{c}\text { Visuelle } \\
\text { Wahrnehmung }\end{array}$ \\
\hline $\begin{array}{l}\text { Goldin et al. } \\
\qquad 2007\end{array}$ & 17 & $17 / 0$ & $\begin{array}{l}\text { negative Filme und } \\
\text { emotionale } \\
\text { Regulation durch } \\
\text { Distanzierung }\end{array}$ & $\begin{array}{c}\text { Neubewertung > } \\
\text { Anschauen }\end{array}$ & 15 & $\begin{array}{c}\text { Visuelle } \\
\text { Wahrnehmung }\end{array}$ \\
\hline
\end{tabular}

\section{Tabelle 2.3: Emotionsregulationsstudien (Fortsetzung)}


Tabelle 2.3: Emotionsregulationsstudien (Fortsetzung)

\begin{tabular}{|c|c|c|c|c|c|c|}
\hline Studie & $\begin{array}{c}\text { Anzahl der } \\
\text { Proban- } \\
\end{array}$ & $\begin{array}{c}\text { Geschlechter- } \\
\text { verteilung }\end{array}$ & Art der & Kontrast & Anzahl & Modalität \\
\hline Studie & $\begin{array}{l}\text { Anzahl der } \\
\text { Proban- } \\
\text { den }\end{array}$ & $\begin{array}{c}\text { Geschlechter- } \\
\text { verteilung } \\
(\mathbf{f} / \mathbf{m})^{*}\end{array}$ & $\begin{array}{c}\text { Art der } \\
\text { Stimulation }\end{array}$ & Kontrast & $\begin{array}{c}\text { Anzahl } \\
\text { Fokusse }\end{array}$ & Modalität \\
\hline $\begin{array}{c}\text { Kross et al. } \\
2009\end{array}$ & 18 & $11 / 7$ & $\begin{array}{c}\text { negative auto- } \\
\text { biographische } \\
\text { Ereignisse und } \\
\text { Regulierung durch } \\
\text { Distanzierung }\end{array}$ & $\begin{array}{l}\text { Akzeptieren >Fühlen } \\
\text { (korrelierte } \\
\text { Aktivierungen bei } \\
\text { der Reduktion } \\
\text { negativer Emotionen) }\end{array}$ & 3 & $\begin{array}{c}\text { Vorwegnehmende } \\
\text { negative } \\
\text { Wahrnehmung }\end{array}$ \\
\hline $\begin{array}{c}\text { Herwig et al. } \\
2807\end{array}$ & 14 & $12 / \Gamma_{0}$ & 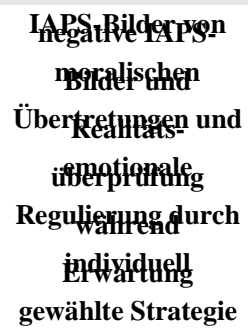 & $\begin{array}{c}\text { Neubeurteilung > } \\
\text { keine Neubeurteilung } \\
\text { Emotionale } \\
\text { (Eryartungshaltung } \\
\text { Regulfation> } \\
\text { negativ >>n } \\
\text { Anschauen } \\
\text { Erwartung neutral) }\end{array}$ & $\mathbf{2}$ & $\begin{array}{c}\text { Visuelle } \\
\text { Visuele } \\
\text { Wahrnehmung }\end{array}$ \\
\hline $\begin{array}{l}\text { McRae et al. } \\
\quad 2008\end{array}$ & 25 & $13 / 12$ & $\begin{array}{c}\text { negative IÂPS- } \\
\text { Bilder und } \\
\text { Regulierung durch } \\
\text { Phantasie oder } \\
\text { Distanzierung }\end{array}$ & $\begin{array}{c}\text { Verminderung> } \\
\text { Anschauen }\end{array}$ & 16 & $\begin{array}{c}\text { Visuelle } \\
\text { Wahrnehmung }\end{array}$ \\
\hline $\begin{array}{l}\text { Kalisch et al. } \\
\text { McRonget al. } \\
2009\end{array}$ & 18 & $\begin{array}{c}8 / 7 \\
18 / 0\end{array}$ & $\begin{array}{c}\text { und } \\
\text { negative IAPS- } \\
\text { vorwegnehmende } \\
\text { Bitier und } \\
\text { Selstablenkung } \\
\text { Regulierung durch } \\
\text { durch Gedanken } \\
\text { Neuinterpretation } \\
\text { anetwas anderes } \\
\text { der Situation }\end{array}$ & $\begin{array}{c}\text { Korrelierte } \\
\text { Aktivierungen bei } \\
\text { Tonische Reaktion } \\
\text { der Reduktion } \\
\text { bei eigener } \\
\text { negativer Emotionen } \\
\text { Ablenkung } \\
\text { und Aktivierung im } \\
\text { Kontrast } \\
\text { (Neubewertung> }\end{array}$ & $\begin{array}{l}1 \\
9\end{array}$ & $\begin{array}{c}\text { Schmerz } \\
\text { Visuelle } \\
\text { Wahrnehmung }\end{array}$ \\
\hline $\begin{array}{l}\text { Modinos et } \\
\text { al. } 2010\end{array}$ & 18 & $7 / 11$ & $\begin{array}{c}\text { IAPS-Bilder von } \\
\text { moralischen } \\
\text { negative IAPS- } \\
\text { Bilder und } \\
\text { emotionale } \\
\text { Regulation durch } \\
\text { Alternativ- } \\
\text { interpretation }\end{array}$ & $\begin{array}{c}\text { Neubewertung> } \\
\text { Anschauen }\end{array}$ & 10 & $\begin{array}{c}\text { Visuelle } \\
\text { Wahrnehmung }\end{array}$ \\
\hline 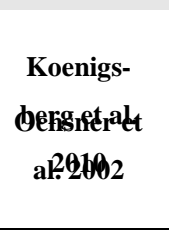 & $\begin{array}{l}16 \\
15\end{array}$ & $\begin{array}{c}9 / 7 \\
15 / 0\end{array}$ & $\begin{array}{c}\text { Bilder (nur soziale } \\
\text { negative IÅS- } \\
\text { Bilder), emotionale } \\
\text { Bilder, emotionale } \\
\text { Regulierung durch } \\
\text { Regulation durch } \\
\text { Distanzierung } \\
\text { Alternativ- }\end{array}$ & $\begin{array}{c}\text { Neubewertung > } \\
\text { Beibehalten } \\
\text { Distanzierung> } \\
\text { und korrelierte } \\
\text { Anschauen } \\
\text { Aktivierungen bei } \\
\text { Reduktion negativer }\end{array}$ & $\begin{array}{c}6 \\
11\end{array}$ & \begin{tabular}{c}
\multicolumn{1}{c}{ Visuelle } \\
Visuefle \\
Wahrnehmung \\
Wahrnehmung
\end{tabular} \\
\hline & & & interpretation & & & \\
\hline
\end{tabular}




\section{Tabelle 2.3: Emotionsregulationsstudien (Fortsetzung)}

* Diese Studien beinhalten 323 weibliche und 104 männliche Probanden.

\begin{tabular}{|c|c|c|c|c|c|c|}
\hline Studie & $\begin{array}{l}\text { Anzahl der } \\
\text { Proban- } \\
\text { den }\end{array}$ & $\begin{array}{c}\text { Geschlechter- } \\
\text { verteilung } \\
(\mathbf{f} / \mathbf{m})^{*}\end{array}$ & $\begin{array}{c}\text { Art der } \\
\text { Stimulation }\end{array}$ & Kontrast & $\begin{array}{l}\text { Anzahl } \\
\text { Fokusse }\end{array}$ & Modalität \\
\hline $\begin{array}{l}\text { Ochsner et } \\
\text { al. } 2004\end{array}$ & 24 & $24 / 0$ & $\begin{array}{c}\text { negative IAPS- } \\
\text { Bilder und } \\
\text { Regulierung durch } \\
\text { Phantasie oder } \\
\text { Distanzierung }\end{array}$ & $\begin{array}{c}\text { Verminderung> } \\
\text { Steigerung }\end{array}$ & 6 & $\begin{array}{c}\text { Visuelle } \\
\text { Wahrnehmung }\end{array}$ \\
\hline $\begin{array}{l}\text { Urry et al. } \\
2006\end{array}$ & 19 & $11 / 8$ & $\begin{array}{l}\text { IAPS-Bilder und } \\
\text { emotionale } \\
\text { Regulation durch } \\
\text { Vorstellungs- } \\
\text { strategie }\end{array}$ & $\begin{array}{c}\text { negative Korrelation } \\
\text { (Verminderung> } \\
\text { Beibehalten) und } \\
\text { Amygdala- } \\
\text { aktivierung }\end{array}$ & 2 & $\begin{array}{c}\text { Visuelle } \\
\text { Wahrnehmung }\end{array}$ \\
\hline $\begin{array}{l}\text { Wager et al. } \\
\qquad 2008\end{array}$ & 30 & $18 / 12$ & $\begin{array}{l}\text { IAPS-Bilder und } \\
\text { emotionale } \\
\text { Regulation durch } \\
\text { Neuinterpretation } \\
\text { der Situation }\end{array}$ & $\begin{array}{c}\text { Korrelierte } \\
\text { Aktivierungen bei } \\
\text { der Reduktion } \\
\text { negativer Emotionen } \\
\text { und Aktivierung im } \\
\text { Kontrast } \\
\text { (Neubewertung> } \\
\text { Anschauen) }\end{array}$ & 8 & $\begin{array}{c}\text { Visuelle } \\
\text { Wahrnehmung }\end{array}$ \\
\hline $\begin{array}{l}\text { Walter et al. } \\
\qquad 2009\end{array}$ & 18 & $18 / 0$ & $\begin{array}{l}\text { IAPS-Bilder und } \\
\text { emotionale } \\
\text { Regulation durch } \\
\text { Distanzierung }\end{array}$ & $\begin{array}{c}\text { Regulation > } \\
\text { keine Regulation }\end{array}$ & 2 & $\begin{array}{c}\text { Visuelle } \\
\text { Wahrnehmung }\end{array}$ \\
\hline $\begin{array}{l}\text { Winecoff et } \\
\text { al. } 2010\end{array}$ & 42 & Keine Angabe & $\begin{array}{l}\text { IAPS-Bilder und } \\
\text { emotionale } \\
\text { Regulation durch } \\
\text { abgetrennte } \\
\text { Perspektive der } \\
\text { Dritten Person }\end{array}$ & $\begin{array}{c}\text { Neubewertung> } \\
\text { Erfahrung }\end{array}$ & 16 & $\begin{array}{c}\text { Visuelle } \\
\text { Wahrnehmung }\end{array}$ \\
\hline
\end{tabular}




\section{Ergebnisse}

Im Folgenden werden die Ergebnisse der Untersuchung über die Gehirnaktivität während der Reduktion negativer Emotionen und der Angstreduktion präsentiert.

\subsection{VMPFC-Aktivität während der Regulation negativer Emotionen}

Wie auch schon im veröffentlichten Artikel von Diekhof, Geier, Falkai und Gruber (Diekhof et al. 2011a) beschrieben, zeigte sich bei der bildlichen Betrachtung der Brennpunkte mit dem Colin T1template (Abbildungen 3.1) eine Gruppierung von aktivierten Arealen im ventromedialen präfrontalen Kortex (VMPFC) bei der Reduktion negativer Emotionen.

Der VMPFC umschreibt eine Region mit inkludiertem medialem orbitofrontalem Kortex, subgenualem und rostralem anteriorem Gyrus cinguli und dem anterioren medialen präfrontalen Kortex (Grabenhorst und Rolls 2011).

Auch die drei unabhängigen domänenspezifischen ALE-Metaanalysen bestätigten diese Übereinstimmung von Aktivierungen im VMPFC (Tabellen 3.1-3.3). Neun Studien, darunter jeweils drei Studien zum Thema „Furchtextinktion“ (Kalisch et al. 2006b, Milad et al. 2007, Schiller et al. 2008), „Placeboeffekt“ (Bingel et al. 2006, Eippert et al. 2009, Watson et al. 2009) und „Emotionsregulation“ (Delgado et al. 2008, Johnstone et al. 2007, Urry et al. 2006), trugen zur Anhäufung der Fokusse in dem ventromedialen präfrontalen Kortex bei. Insgesamt entstand dieses Ergebnis aus 15 relevanten Fokussen, die sich aus vier Fokussen aus den drei Studien über die Furchtextinktion, vier Fokussen aus den drei Emotionsregulationsstudien und sieben Fokussen aus den drei Studien über die Placebobehandlung zusammensetzten. In 17 der 45 Studien zeigte sich eine verstärkter Aktivierung im Bereich des VMPFC mit angrenzendem medialem OFC, subgenualem und rostralem ACC und medialem PFC (Furchtextinktionsstudien: Finger et al. 2008, Kalisch et al. 2006b, Milad et al. 2007, Schiller et al. 2008, Soliman et al. 2010, Placebostudien: Bingel et al. 2006, Diekhof et al. 2011b, Eippert et al. 2009, Kong et al. 2006, Petrovic et al. 2005, Scott et al. 2008, Watson et al. 2009, Emotionsregulationsstudien: Banks et al. 2007, Delgado et al. 2008, Johnstone et al. 2007, Mak et al. 2009, Urry et al. 2006). 
Abbildung 3.1: Gehirnaktivierung bei Furchtextinktionsstudien (Rot), Placebostudien (Blau), Emotionsregulationsstudien (Grün). Schnitte des Colin T1-template im MNIKoordinatenraum. Übereinstimmende Aktivierung im VMPFC durch Fadenkreuz gekennzeichnet. Skala zeigt ALE-Werte an.

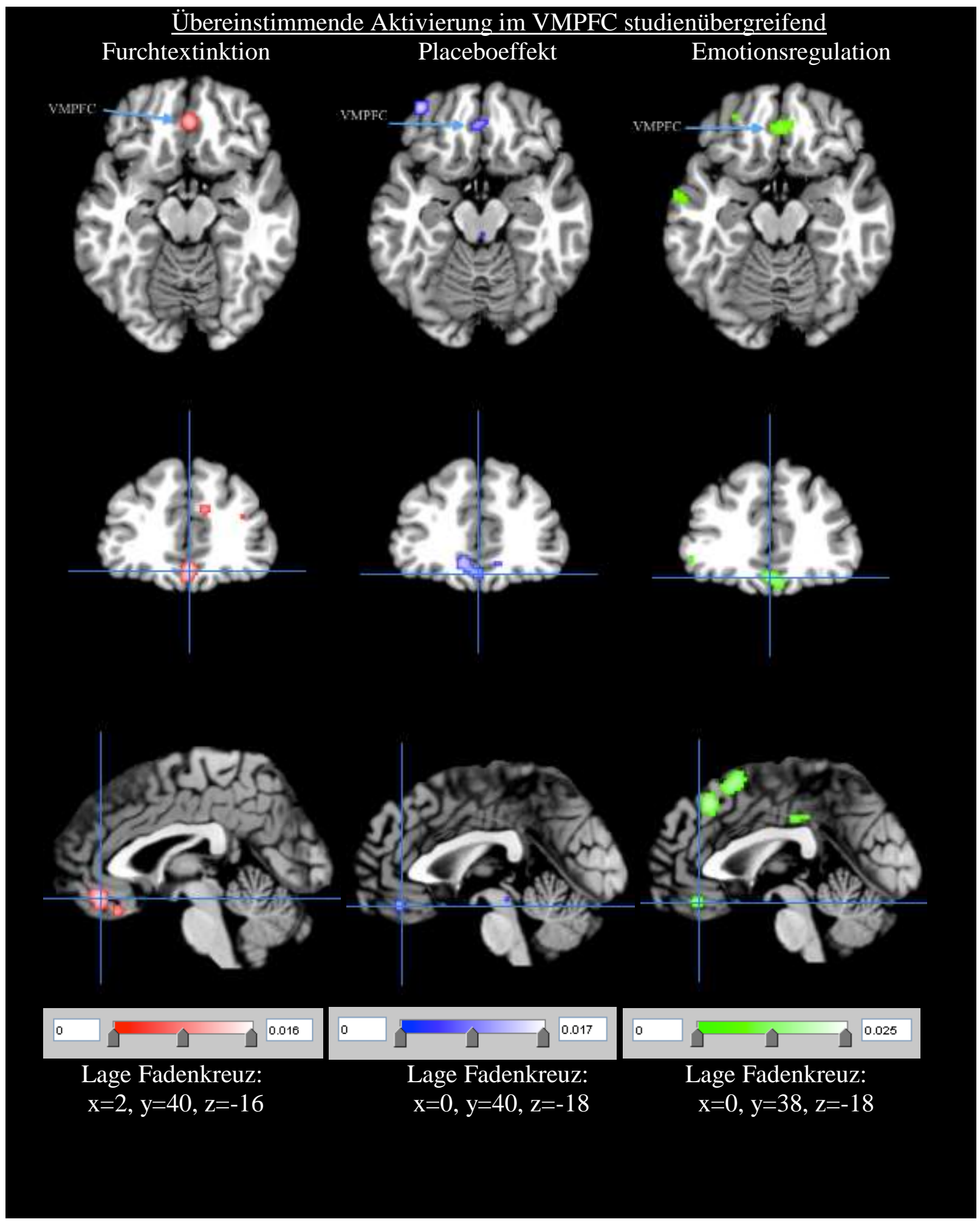




\subsection{Regionen mit verstärkter Aktivität neben dem VMPFC}

Neben dem VMPFC gab es noch weitere studientypspezifische Gehirnregionen, in denen vermehrte Überlappungen von aktivierten Fokussen sichtbar wurden.

Bei der Metaanalyse mit den Koordinaten der Furchtextinktionsstudien zeigten sich vier weitere Gehirnareale mit konkordanten Gehirnaktivierungen (Tabelle 3.1). Unter diesen Gehirnaktivierungen waren der rechte subgenuale anteriore Gyrus cinguli (sgACC) sowie der rechte mediale Bereich des Gyrus cinguli. Zusätzlich wurden noch der linke Paraphippocampus und die rechte Uvula vermis des Cerebellums mit verstärkter Aktivität auffällig, jedoch nur mit geringeren Anzahl an aktivierten Fokussen bzw. geringerer Clustergröße (L Parahippocampus ALE-Wert: 0.0090; R Uvula ALE-Wert: 0.0092).

Tabelle 3.1: ALE-Metaanalyse der Gehirnaktivierungen in Furchtextinktionsstudien

\begin{tabular}{|c|c|c|c|c|c|c|c|}
\hline \multirow{2}{*}{ Region } & \multicolumn{3}{|c|}{$\begin{array}{c}\text { MNI- } \\
\text { Koordinaten }\end{array}$} & \multirow{2}{*}{$\begin{array}{l}\text { ALE- } \\
\text { Wert }\end{array}$} & \multirow{2}{*}{$\begin{array}{c}\text { Clustergröße } \\
\left(\mathbf{m m}^{\wedge} \mathbf{3}\right)\end{array}$} & \multirow{2}{*}{$\begin{array}{l}\text { Anzahl der Fokusse innerhalb des } \\
\text { Clusters }\end{array}$} & \multirow{2}{*}{$\begin{array}{c}\text { Anzahl der } \\
\text { Studien }\end{array}$} \\
\hline & $\mathbf{x}$ & $\mathbf{y}$ & $\mathbf{z}$ & & & & \\
\hline \multirow[b]{2}{*}{ R VMPFC * } & 2 & 40 & -16 & 0.0134 & \multirow[b]{2}{*}{832} & \multirow[b]{2}{*}{4} & \multirow[b]{2}{*}{3} \\
\hline & 6 & 50 & & 0.0085 & & & \\
\hline R sgACC & 8 & 26 & -22 & 0.0123 & 584 & 3 & 2 \\
\hline R Gyrus cinguli & 12 & 36 & 22 & 0.0157 & 560 & 2 & 1 \\
\hline $\begin{array}{c}\mathrm{L} \\
\text { Parahippocampus }\end{array}$ & -26 & -18 & -26 & 0.0090 & 184 & 2 & 1 \\
\hline R Uvula & 12 & -80 & -36 & 0.0092 & 176 & 2 & 1 \\
\hline
\end{tabular}

Diese Metaanalyse beinhaltet Koordinaten von 9 Studien mit 158 Probanden und insgesamt 53 Fokusse.

* Cluster beinhaltet die Studien Kalisch et al. 2006b, Milad et al. 2007, Schiller et al. 2008

Weiterhin zeigte die Metaanalyse über die Placebostudien acht weitere Gehirnregionen mit konkordanten Aktivierungen auf (Tabelle 3.2). Unter diesen Regionen waren der mittlere frontale Gyrus, linker und rechter Pars anterior des Gyrus cinguli (ACC) mit dem angrenzenden mittleren Gyrus cinguli, der rechte mediale frontale Gyrus und der Sulkus frontomarginalis, rechter und linker inferiorer frontaler Gyrus mit angrenzender Inselrinde rechts und sgACC links sowie 
linkseitig der Pars anterior des inferioren frontalen Gyrus, rechter und linker anteriorer orbitofrontaler Kortex (OFC) und der rechte superiore frontale Sulkus.

Tabelle 3.2: ALE-Metaanalyse der Gehirnaktivierungen in Placebostudien

\begin{tabular}{|c|c|c|c|c|c|c|c|}
\hline \multirow{2}{*}{ Region } & & MNI & & ALE- & \multirow{2}{*}{$\begin{array}{c}\text { Clustergröße } \\
\left(\mathbf{m m}^{\wedge} \mathbf{3}\right)\end{array}$} & \multirow{2}{*}{$\begin{array}{c}\text { Anzahl der Fokusse } \\
\text { innerhalb des Clusters }\end{array}$} & \multirow{2}{*}{$\begin{array}{c}\text { Anzahl der } \\
\text { Studien }\end{array}$} \\
\hline & $\mathbf{x}$ & $\mathbf{y}$ & $\mathbf{z}$ & & & & \\
\hline \multirow{4}{*}{ L/R VMPFC } & -8 & 40 & -12 & 0.0133 & & & \\
\hline & & & & & 656 & 5 & $3 *$ \\
\hline & O & 40 & -18 & 0.0118 & & & \\
\hline & 14 & 36 & -12 & 0.0131 & 232 & 2 & $2 * *$ \\
\hline R Mittlerer frontaler Gyrus & 42 & 6 & 30 & 0.0171 & 472 & 3 & 2 \\
\hline $\begin{array}{c}\text { R (anteriorer) mittlerer Gyrus } \\
\text { cinguli }\end{array}$ & 10 & 12 & 32 & 0.0140 & 552 & 4 & 4 \\
\hline L ACC & -6 & 28 & 26 & 0.0145 & 416 & 3 & 3 \\
\hline $\begin{array}{l}\text { R medialer frontaler Gyrus/ } \\
\text { fontomarginaler Sulkus }\end{array}$ & 16 & 50 & 12 & 0.0151 & 608 & 3 & 2 \\
\hline $\begin{array}{l}\text { R medialer frontaler Gyrus/ } \\
\text { fontomarginaler Sulkus }\end{array}$ & 30 & 48 & 8 & 0.0140 & 552 & 3 & 3 \\
\hline $\begin{array}{c}\text { R inferiorer frontaler Gyrus/ } \\
\text { anteriore Inselrinde }\end{array}$ & 46 & 20 & -4 & 0.0146 & 280 & 2 & 1 \\
\hline $\begin{array}{l}\text { L inferiorer frontaler Gyrus, } \\
\text { sgACC }\end{array}$ & -8 & 10 & -12 & 0.0135 & 248 & 2 & 2 \\
\hline $\begin{array}{c}\mathrm{L} \text { anteriorer inferiorer frontaler } \\
\text { Gyrus }\end{array}$ & -40 & 52 & 6 & 0.0131 & 296 & 3 & 2 \\
\hline $\mathrm{R}$ anteriorer $\mathrm{OFC}$ & 28 & 48 & -10 & 0.0144 & 312 & 2 & 2 \\
\hline $\mathrm{L}$ anteriorer $\mathrm{OFC}$ & -38 & 52 & -18 & 0.0152 & 272 & 2 & 1 \\
\hline R superiorer frontaler Sulkus & 22 & 14 & 38 & 0.0148 & 240 & 2 & 1 \\
\hline
\end{tabular}

Diese Metaanalyse beinhaltet Koordinaten von 13 Studien mit 239 Probanden und insgesamt 119 Fokusse.

*Cluster beinhaltet die Studien Bingel et al. 2006, Eippert et al. 2009, Watson et al. 2009 ** Cluster beinhaltet die Studien Eippert et al. 2009, Watson et al. 2009

In der Metaanalyse über die willentliche Emotionsregulation stellten sich schließlich 13 weitere Gehirnregionen mit Überlappungen von Aktivierungsfokussen heraus (Tabelle 3.3). Zu diesen 
Regionen zählten der linke dorsomediale präfrontale Kortex mit dem angrenzenden Pars anterior des Gyrus cinguli und der mediale frontale Gyrus, der rechte und linke mittlere frontale Gyrus mit angrenzendem DLPFC, der rechte und linke inferiore frontale Kortex bis hin zur Inselrinde, der rechte und linke intraparietale Kortex, der linke inferiore temporale Sulkus, das rechte und linke Operkulum mit angrenzender anteriorer Inselrinde, der linke mittlere temporale Kortex, der rechte frontomarginale Sulkus und LPFC und der rechte superiore frontale Gyrus. Noch weitere fünf Gehirnareale zeigten konkordante Aktivierungen, jedoch mit geringerer Clustergröße. Dazu zählten der rechte (ALE-Wert: 0.0127) und linke Gyrus cinguli (ALE-Werte: 0.0138, 0.0118, 0.0107), der rechte Nucleus caudatus (ALE-Wert: 0.0153) und der rechte (ALE-Wert: 0.0127) und linke superiore temporale Gyrus (ALE-Wert: 0.0141).

Tabelle 3.3: ALE-Metaanalyse der Gehirnaktivierungen in Emotionsregulationsstudien.

\section{Regionen mit geringerer Clustergröße abgegrenzt am Ende der Tabelle dargestellt.}

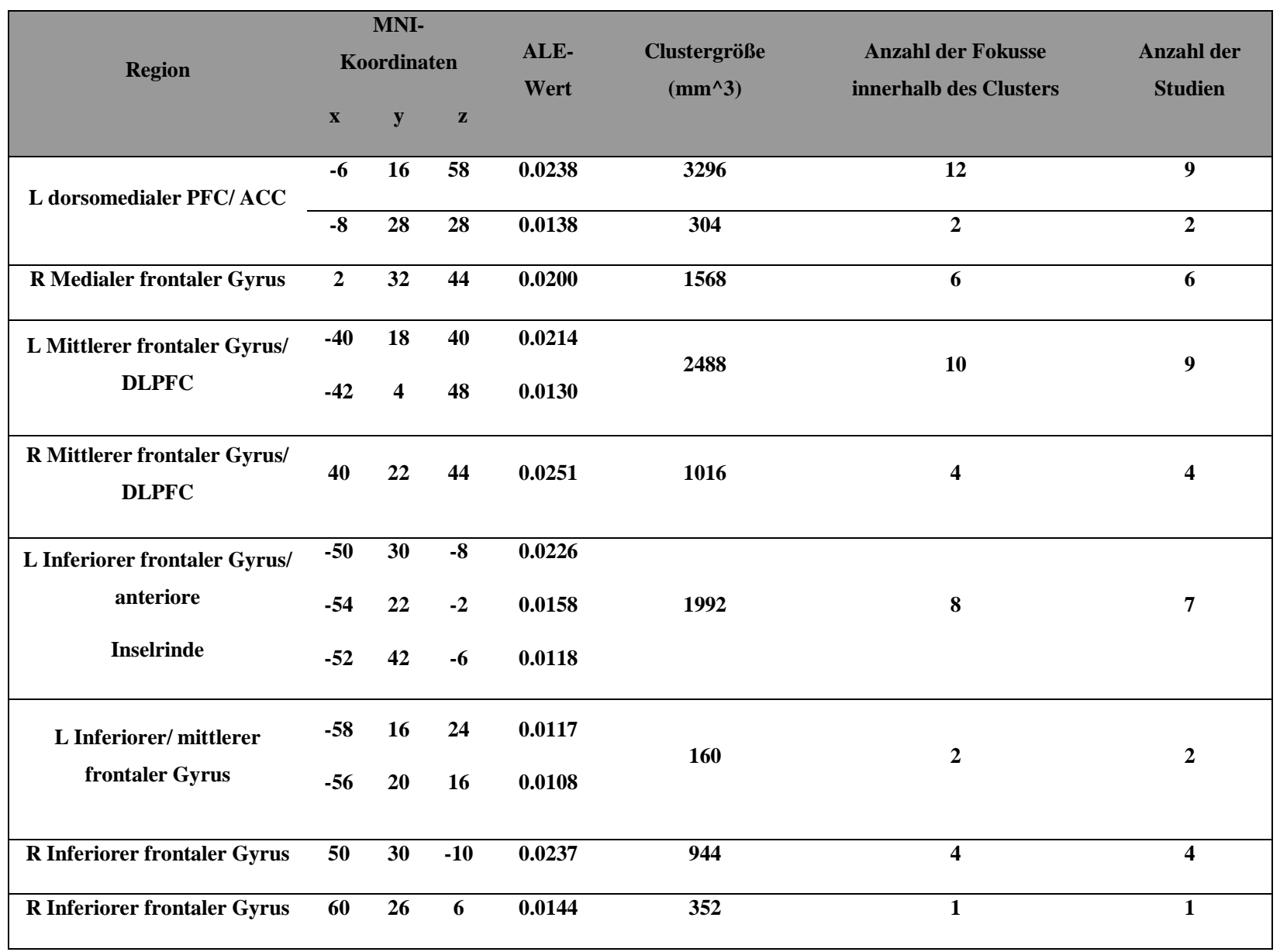


Tabelle 3.3: ALE-Metaanalyse der Gehirnaktivierungen in Emotionsregulationsstudien. Regionen mit geringerer Clustergröße abgegrenzt am Ende der Tabelle dargestellt. (Fortsetzung)

\begin{tabular}{|c|c|c|c|c|c|c|c|}
\hline \multirow{2}{*}{ Region } & \multicolumn{3}{|c|}{$\begin{array}{c}\text { MNI- } \\
\text { Koordinaten }\end{array}$} & \multirow{2}{*}{$\begin{array}{l}\text { ALE- } \\
\text { Wert }\end{array}$} & \multirow{2}{*}{$\begin{array}{c}\text { Clustergröße } \\
\left(\mathbf{m m}^{\wedge} \mathbf{3}\right)\end{array}$} & \multirow{2}{*}{$\begin{array}{l}\text { Anzahl der Fokusse innerhalb } \\
\text { des Clusters }\end{array}$} & \multirow{2}{*}{$\begin{array}{c}\text { Anzahl der } \\
\text { Studien }\end{array}$} \\
\hline & $\mathbf{x}$ & $\mathbf{y}$ & $\mathbf{z}$ & & & & \\
\hline \multirow{3}{*}{ L Intraparietaler Kortex } & -46 & -66 & 36 & 0.0202 & \multirow{3}{*}{1840} & \multirow{3}{*}{8} & \multirow{3}{*}{6} \\
\hline & -42 & -56 & 38 & 0.0137 & & & \\
\hline & -38 & -62 & 30 & 0.0125 & & & \\
\hline R Intraparietaler Kortex & 50 & -58 & 42 & 0.0159 & 384 & 3 & 3 \\
\hline $\begin{array}{c}\text { L Inferiorer temporaler } \\
\text { Sulkus }\end{array}$ & -62 & -36 & -2 & 0.0222 & 1368 & 5 & 4 \\
\hline $\mathbf{R}$ anteriore Inselrinde & 46 & 14 & $\mathbf{0}$ & 0.0171 & 1256 & 6 & 4 \\
\hline $\mathrm{L}$ anteriore Inselrinde & -38 & 20 & -4 & 0.0130 & 288 & 2 & 2 \\
\hline L/ R VMPFC & $\begin{array}{l}6 \\
0\end{array}$ & $\begin{array}{l}40 \\
38\end{array}$ & $\begin{array}{l}-22 \\
-18\end{array}$ & $\begin{array}{l}0.0162 \\
0.0144\end{array}$ & 656 & 4 & 3* \\
\hline $\begin{array}{c}\text { L Mittlerer temporaler } \\
\text { Gyrus }\end{array}$ & -64 & -4 & -22 & 0.0153 & 384 & 3 & 3 \\
\hline $\begin{array}{c}\text { R Frontomarginaler Sulkus/ } \\
\text { LPFC }\end{array}$ & 34 & 60 & 8 & 0.0157 & 368 & 3 & 3 \\
\hline R Superiorfrontaler Gyrus & 18 & 24 & 58 & 0.0127 & 240 & 3 & 2 \\
\hline \multirow[b]{2}{*}{ L Gyrus cinguli } & -8 & 28 & 28 & 0.0138 & 304 & 2 & 2 \\
\hline & $\begin{array}{l}0 \\
0\end{array}$ & $\begin{array}{l}-24 \\
-30\end{array}$ & $\begin{array}{l}34 \\
36\end{array}$ & $\begin{array}{l}0.0118 \\
0.0107\end{array}$ & 176 & 3 & 3 \\
\hline R Gyrus cinguli & 6 & 20 & 22 & 0.0127 & 160 & 2 & 1 \\
\hline R Nucleus caudatus & 20 & 14 & 20 & 0.0153 & 256 & 2 & 2 \\
\hline $\begin{array}{c}\text { L Superiorer temporaler } \\
\text { Gyrus }\end{array}$ & -62 & -52 & 20 & 0.0141 & 192 & 2 & 2 \\
\hline $\begin{array}{c}\text { R Superiorer temporaler } \\
\text { Gyrus }\end{array}$ & 56 & -56 & 28 & 0.0127 & 128 & 2 & 2 \\
\hline
\end{tabular}

Diese Metaanalyse beinhaltet Koordinaten von 23 Studien mit 478 Probanden und insgesamt 196 Fokusse.

* Cluster beinhaltet die Studien Delgado et al. 2008, Johnstone et al. 2007, Urry et al. 2006 


\subsection{Gehirnaktivierungen bei der Emotionsregulation - Beschränkung auf Studien mit ähnlicher Verteilung an weiblichen und männlichen Probanden}

Eine weitere ALE- Metaanalyse über die Emotionsregulation, deren inkludierte Studien anteilig ungefähr gleich viele weibliche als auch männliche Probanden beinhaltete, bestätigte die meisten der aktivierten Gehirnregionen der Analyse der Emotionsregulationsstudien mit allen Studien, einschließlich der Anhäufung im VMPFC (Tabelle 3.4). Diese Analyse wurde durchgeführt, da einige der Emotionsregulationsstudien der allgemeinen Metaanalyse nur mit Frauen durchgeführt wurden und diese möglicherweise stärkere Emotionen zeigen, die folglich eine stärkere emotionale Regulation erfordern (z.B. Drabant et al. 2008, Eippert et al. 2007, Goldin et al.2007).

Tabelle 3.4: ALE-Metaanalyse der Gehirnaktivierungen in Emotionsregulationsstudien $\underline{\text { (Studien mit ähnlicher Anzahl an Männern und Frauen) }}$

\begin{tabular}{|c|c|c|c|c|c|c|c|}
\hline Region & $\begin{array}{r}\text { MNI } \\
\mathbf{x}\end{array}$ & $\overline{\text { Koorc }}$ & Iaten & $\begin{array}{l}\text { ALE- } \\
\text { Wert }\end{array}$ & $\begin{array}{c}\text { Clustergröße } \\
\left(\mathbf{m m}^{\wedge} \mathbf{3}\right)\end{array}$ & $\begin{array}{c}\text { Anzahl der Fokusse } \\
\text { innerhalb des Clusters }\end{array}$ & $\begin{array}{c}\text { Anzahl der } \\
\text { Studien }\end{array}$ \\
\hline L intraparietaler Kortex & $\begin{array}{l}-46 \\
-42\end{array}$ & $\begin{array}{l}-66 \\
-56\end{array}$ & $\begin{array}{l}36 \\
38\end{array}$ & $\begin{array}{l}0.0200 \\
0.0136\end{array}$ & 2152 & 8 & 6 \\
\hline $\mathbf{R}$ intraparietaler Kortex & 50 & -58 & 42 & 0.0159 & 552 & 3 & 3 \\
\hline L dorsomedialer PFC/ ACC & $\begin{array}{l}-10 \\
-6\end{array}$ & $\begin{array}{l}22 \\
16\end{array}$ & $\begin{array}{l}60 \\
58\end{array}$ & $\begin{array}{l}0.0151 \\
0.0148\end{array}$ & 1104 & 5 & 5 \\
\hline $\begin{array}{c}\text { L inferiorer frontaler Gyrus/ } \\
\text { anteriore Inselrinde }\end{array}$ & $\begin{array}{l}-54 \\
-52\end{array}$ & $\begin{array}{l}20 \\
30\end{array}$ & $\begin{array}{l}0 \\
-8\end{array}$ & $\begin{array}{l}0.0152 \\
0.0137\end{array}$ & 912 & 4 & 4 \\
\hline $\mathbf{R}$ inferiorer frontaler Gyrus & 52 & 30 & -8 & 0.0204 & 744 & 3 & 3 \\
\hline $\begin{array}{l}\text { R anteriore Inselrinde, } \\
\text { frontales Operkulum }\end{array}$ & $\begin{array}{l}46 \\
42\end{array}$ & $\begin{array}{l}16 \\
20\end{array}$ & $\begin{array}{l}4 \\
-4\end{array}$ & $\begin{array}{l}0.0141 \\
0.0107\end{array}$ & 912 & 5 & 3 \\
\hline L medialer frontaler Gyrus/ & $\begin{array}{l}-42 \\
-42\end{array}$ & $\begin{array}{l}4 \\
12\end{array}$ & $\begin{array}{l}48 \\
50\end{array}$ & $\begin{array}{l}0.0128 \\
0.0122\end{array}$ & 896 & 5 & 5 \\
\hline (R) VMPFC & $\begin{array}{l}6 \\
0\end{array}$ & $\begin{array}{l}40 \\
28\end{array}$ & $\begin{array}{l}-22 \\
-18\end{array}$ & $\begin{array}{l}0.0162 \\
0.0144\end{array}$ & 856 & 4 & $3 *$ \\
\hline $\mathrm{L}$ inferiorer temporaler Sulkus & -62 & -36 & -4 & 0.0146 & 552 & 3 & 3 \\
\hline Posteriorer Gyrus cinguli & $\begin{array}{l}\mathbf{0} \\
\mathbf{0}\end{array}$ & $\begin{array}{l}-24 \\
-30\end{array}$ & $\begin{array}{l}34 \\
36\end{array}$ & $\begin{array}{l}0.0118 \\
0.0107\end{array}$ & 400 & 3 & 3 \\
\hline
\end{tabular}


Tabelle 3.4: ALE-Metaanalyse der Gehirnaktivierungen in Emotionsregulationsstudien (Studien mit ähnlicher Anzahl an Männern und Frauen) (Fortsetzung)

\begin{tabular}{|c|c|c|c|c|c|c|c|}
\hline \multirow{2}{*}{ Region } & \multicolumn{3}{|c|}{ MNI- Koordinaten } & \multirow{2}{*}{$\begin{array}{l}\text { ALE- } \\
\text { Wert }\end{array}$} & \multirow{2}{*}{$\begin{array}{c}\text { Clustergröße } \\
\left(\mathbf{m m}^{\wedge} \mathbf{3}\right)\end{array}$} & \multirow{2}{*}{$\begin{array}{l}\text { Anzahl der Fokusse } \\
\text { innerhalb des Clusters }\end{array}$} & \multirow{2}{*}{$\begin{array}{c}\text { Anzahl der } \\
\text { Studien }\end{array}$} \\
\hline & $\mathbf{x}$ & $\mathbf{y}$ & $\mathbf{z}$ & & & & \\
\hline $\mathbf{R}$ inferiorer parietaler Kortex & 56 & -56 & 28 & 0.0123 & 240 & 2 & 2 \\
\hline R Nucleus Caudatus & 20 & 14 & 20 & 0.0153 & 344 & 2 & 2 \\
\hline L mittlerer temporaler Gyrus & -60 & -6 & -20 & 0.011 & 160 & 2 & 2 \\
\hline \multirow{3}{*}{ L Inselrinde/ Claustrum } & -38 & 20 & -2 & 0.0104 & \multirow{3}{*}{152} & \multirow{3}{*}{2} & \multirow{3}{*}{2} \\
\hline & & & & & & & \\
\hline & -36 & 16 & 2 & 0.0103 & & & \\
\hline
\end{tabular}

Diese Metaanalyse beinhaltet Koordinaten von 13 Studien mit 274 Probanden und insgesamt 99 Fokusse.

* Cluster beinhaltet die Studien Delgado et al. 2008, Johnstone et al. 2007, Urry et al. 2006

\subsection{Zentrum der Überlappung von Aktivität}

Die abschließende Metaanalyse von allen Studientypen unabhängig von dem experimentellen Design sowie die visuelle Darstellung der Ergebnisse der drei Metaanalysen mit der Software Mango zeigte ein Zentrum der Überlappungen der Brennpunkte im VMPFC mit zwei Clustermaxima (MNI-Koordinaten: 0,40,-18, ALE-Wert: 0.0372, Clustergröße: 3024 mm^3 und MNI-Koordinaten: 6,26,-22; ALE-Wert: 0.0198, Clustergröße: 3024 mm^3). Die Berechnung der größten Überlappung von Gehirnaktivität aus den neun Studien, die direkt zum Ergebnis des VMPFC betrugen, ergab das Zentrum der stärksten Aktivität mit den MNI- Koordinaten 2, 40,-18 mit dem ALE-Wert 0,0274 und der Clustergröße von 1040 mm^3.

\subsection{Paarmetaanalyse mit Emotionsregulations- und Placebostudien}

Es wurde noch eine weitere Analyse durchgeführt, um Überlappungen von Gehirnaktivierungen zwischen den zwei Studientypen „Emotionsregulation und Placeboeffekt" herauszufinden. Grundlage dieser Untersuchung war der Gedanke, dass sich diese beiden Studientypen besonders 
ähnelten, da sie im Vergleich zur Furchtextinktion geistig anspruchsvoller sind und der negative Reiz permanent dargeboten wird.

Die Metaanalyse deckte neben dem VMPFC zwei weitere Gehirnregionen mit verstärkter Aktivierung auf. Eine Häufung der Aktivierungspunkte zeigte sich in der rechten anterioren Inselrinde (MNI-Koordinaten: 46,18,-4; ALE-Wert: 0.0257; Clustergröße: 2864 mm^3). Zusätzlich fiel noch der linke anteriore Gyrus cinguli (MNI-Koordinaten: -6,28,26; ALEWert:0.026; Clustergröße:1216 mm^3) auf (Abbildung 3.2). 
Abbildung 3.2: Paarmetaanalyse: Verstärkte Aktivierungen in der rechten Inselrinde und dem linken ACC bei Emotionsregulation- und Placebostudien. Gehirnschnitte des Colin T1template im MNI- Koordinatenraum.

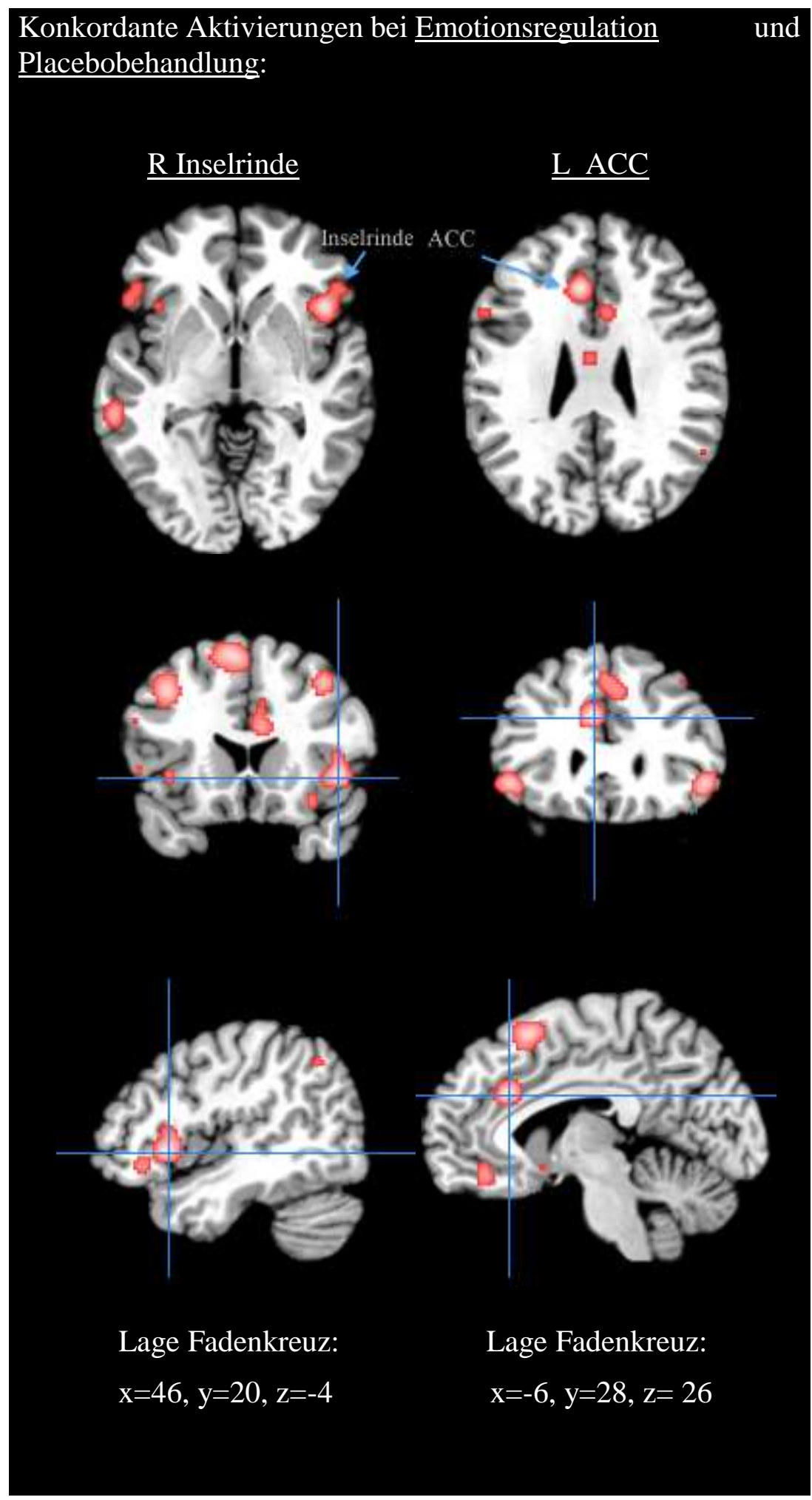




\subsection{Amygdala-Deaktivierung während der Reduktion negativer Emotionen}

Schließlich wurde untersucht, ob die verstärkte Aktivierung im VMPFC während der Angstreduktion von einer verminderten Aktivierung in einer anderen Gehirnregion begleitet war. Daraus ließe sich schließen, dass diese Regionen mit reduzierter Gehirnaktivität während der Regulation negativer Emotionen eine zentrale Rolle bei der Wahrnehmung von negativen Emotionen und insbesondere von Angst spielen. Auffällig war, dass viele Studien während der Regulation negativer Emotionen durch Furchtextinktion, Placebobehandlung oder willentliche Emotionsregulation von einer verminderten Aktivität in der Amygdala berichteten. Dies ließ eine funktionelle Verbindung zwischen dem VMPFC und der Amygdala vermuten.

Nicht alle Studien, die in die Metaanalysen miteinbezogen wurden untersuchten auch Gehirndeaktivierungen. Vier der neun Furchtextinktionsstudien berichteten von verminderten Aktivierungen während des Vorgangs, die nicht unbedingt auf die Amygdala beschränkt waren (Finger et al. 2008, Knight et al. 2004, Soliman et al. 2010, Spoormaker et al. 2010). Bei den Studien über den Placeboeffekt waren es 10 von 13 Studien, die Deaktivierungen auch außerhalb der Amygdala berichteten (Bingel et al. 2006, Diekhof et al. 2011b, Eippert et al. 2009, Kong et al. 2006, Kong et al. 2009, Petrovic et al. 2005, Sarinopoulos et al. 2006, Wager et al. 2004 (1),(2), Watson et al. 2009). Ebenso zählten 15 der 23 Emotionsregulationsstudien dazu, die von Gehirndeaktivierungen während der Regulation negativer Emotionen berichteten (Delgado et al. 2008, Eippert et al. 2007, Goldin et al. 2007, Herwig et al. 2007, Johnstone et al. 2007, Kalisch et al. 2006c, Koenigsberg et al. 2010, Kross et al. 2009, Mak et al. 2009, McRae et al. 2009, Ochsner et al. 2002, 2004, Urry et al. 2006, Walter et al. 2009, Winecoff et al. 2010). Insgesamt waren es 29 Studien aus allen drei Studientypen, die in die Berechnungen mit verminderter Gehirnaktivierung bei der Regulation negativer Emotionen miteinflossen. Von diesen Studien berichteten drei der Furchtextinktionsstudien über verminderte Aktivität im Bereich der Amygdala während der Angstreduktion (Knight et al. 2004, Soliman et al. 2010, Spoormaker et al. 2010) (Tabelle 8.1). Bei den Placebostudien zeigten vier Studien Gehirndeaktivierungen in der Amygdalaregion (Bingel et al. 2006, Eippert et al. 2009; Petrovic et al. 2005, Sarinopoulos et al. 2006) (Tabelle 8.2) und bei den Emotionsregulationsstudien waren es 11 Studien, die auf verminderte Aktivierungen der Amygdala hinwiesen (Delgado et al. 2008, Eippert et al. 2007, Goldin et al. 2007, Herwig et al. 2007, Johnstone et al. 2007, Koenigsberg et al. 2010, McRae et al. 2009, Ochsner et al. 2004, Urry et al. 2006, Walter et al. 2009, Winecoff et al. 2010) (Tabelle 
8.3). Folglich ist festzustellen, dass $\mathbf{1 8}$ der 29 Studien, die von verminderten Gehirnaktivitäten während der Regulation negativer Emotionen berichteten, verminderte Aktivität unter anderem speziell in der Amygdala herausfanden. In den Studien Sarinopoulos et al. (2006) und Johnstone et al. (2007) wurde von Deaktivierungen im Amygdalabereich berichtet, was jedoch die Analyse mit Ginger ALE 2.0 nicht zeigte. Dies lag möglicherweise an verminderten Aktivierungen einer anderen Hemisphäre oder in einem anderen Amygdalabereich, sodass Ginger ALE diese Bereiche fälschlicherweise nicht erfasste.

Weiterhin wurden aus den 29 Studien, die von verminderten Aktivierungen in Gehirnbereichen während der Gefühlsregulation berichteten, 11 auffällig durch verstärkte Aktivität im VMPFCBereich. Dies waren zwei Furchtextinktionsstudien (Soliman et al. 2010, Finger et al. 2008), fünf Placebostudien (Bingel et al. 2006, Diekhof et al. 2011b, Eippert et al. 2009, Petrovic et al. 2005, Watson et al. 2009), und vier Emotionsregulationsstudien (Delgado et al. 2008, Johnstone et al. 2007, Mak et al. 2009, Urry et al. 2006). Somit war festzustellen, dass 7 der 29 Studien gleichzeitig von VMPFC-Aktivierungen und einer Reduktion der Aktivität im Bereich der Amygdala während der Regulation negativer Emotionen und im Besonderen während der Angstreduktion berichteten (Soliman et al. 2010, Bingel et al. 2006, Petrovic et al. 2005, Eippert et al. 2009, Urry et al. 2006, Delgado et al. 2008, Johnstone er al. 2007). Daraus war zu schlussfolgern, dass in 64\% (7/11=0.64) der Studien, die über eine VMPFC-Aktivierung während der Reduktion negativer Emotionen berichteten, auch eine Amygdala-Deaktivität festgestellt wurde. Jedoch ist zu erwähnen, dass von den 29 Studien, die auch die reduzierten Gehirnaktivitäten während der Reduktion negativer Emotionen untersuchten, nur sieben Studien eine VMPFC-Aktivierung mit gleichzeitiger Amygdala-Deaktivität feststellten, was lediglich 24\% ergibt.

Desweitern zeigten die Analysen über die Gehirndeaktivierungen während der Regulation negativer Emotionen nur noch Gehirnregionen mit kleinen Clustern an Deaktivierungen, die sich maximal aus Fokussen von zwei Studien zusammensetzten (Tabellen 8.1-8.3). 
Tabelle 3.5: ALE-Metaanalyse der Gehirndeaktivierungen in allen Studientypen $\underline{\text { (Furchtextinktions-, Placebo-, Emotionsregulationsstudien) }}$

\begin{tabular}{|c|c|c|c|c|c|c|c|}
\hline \multirow{2}{*}{ Region } & \multicolumn{3}{|c|}{ Koordinaten } & \multirow{2}{*}{$\begin{array}{l}\text { ALE- } \\
\text { Wert }\end{array}$} & \multirow{2}{*}{$\begin{array}{c}\text { Clustergröße } \\
\left(\mathbf{m m}^{\wedge} \mathbf{3}\right)\end{array}$} & \multirow{2}{*}{$\begin{array}{l}\text { Anzahl der Fokusse innerhalb } \\
\text { des Clusters }\end{array}$} & \multirow{2}{*}{$\begin{array}{c}\text { Anzahl der } \\
\text { Studien }\end{array}$} \\
\hline & $\mathbf{x}$ & $\mathbf{y}$ & $\mathbf{z}$ & & & & \\
\hline & & & & & 4832 & & \\
\hline L Amygdala & -26 & -2 & -22 & 0.0443 & & 18 & $15^{*}$ \\
\hline \multirow{3}{*}{ R Amygdala } & 26 & -4 & -14 & 0.0175 & & & \\
\hline & 22 & $\mathbf{0}$ & -24 & 0.0164 & 2464 & 10 & $8^{*}$ \\
\hline & 36 & 2 & -24 & 0.0137 & & & \\
\hline \multirow{3}{*}{ L Inselrinde } & -36 & 2 & -4 & 0.0171 & 1232 & 6 & 4 \\
\hline & -44 & -16 & $\mathbf{0}$ & 0.0185 & 760 & 3 & 3 \\
\hline & -40 & -24 & 20 & 0.0134 & 224 & 2 & 2 \\
\hline R Inselrinde & 40 & 6 & $\mathbf{0}$ & 0.0160 & 352 & 2 & 2 \\
\hline L Postzentraler Gyrus & -58 & -24 & 24 & 0.0206 & 1008 & 4 & 3 \\
\hline R Postzentraler Gyrus & 58 & -24 & 22 & 0.0129 & 288 & 2 & 2 \\
\hline L ACC & $\mathbf{0}$ & 28 & 22 & 0.0194 & 792 & 4 & 3 \\
\hline & & & & & 752 & & \\
\hline R Thalamus & 12 & -4 & 14 & 0.0176 & & 4 & 3 \\
\hline R Okzipitaler Kortex & 4 & -86 & 2 & 0.0135 & 256 & 2 & 1 \\
\hline R parietaler Kortex & 42 & -42 & 48 & 0.0132 & 256 & 2 & 2 \\
\hline $\begin{array}{c}\text { Inferiorer frontaler } \\
\text { Gyrus }\end{array}$ & -42 & 24 & -26 & 0.0143 & 200 & 2 & 2 \\
\hline
\end{tabular}

Diese Metaanalyse beinhaltete Koordinaten von 29 Studien mit 557 Probanden und insgesamt 182 Fokusse.

*Bingel et al. 2006, Delgado et al. 2008, Eippert et al. 2007, Eippert et al. 2009, Goldin et al. 2007, Herwig et al. 2007, Knight et al. 2004, McRae et al. 2009, Ochsner et al. 2004, Petrovic et al. 2005, Solimann et al. 2010, Spoormaker et al. 2010, Urry et al. 2006, Walter et al. 2009, Winecoff et al. 2010

**Eippert et al. 2009, Koenigsberg et al. 2010, McRae et al. 2009, Ochsner et al. 2004, Petrovic et al. 2005, Urry et al. 2006, Walter et al. 2009, Winecoff et al. 2010 
Abbildung 3.3: Maximum der geringsten Aktivierung in der Amygdala in allen drei Studientypen. Gehirnschnitte des Colin T1-template im MNI-Koordinatenraum.

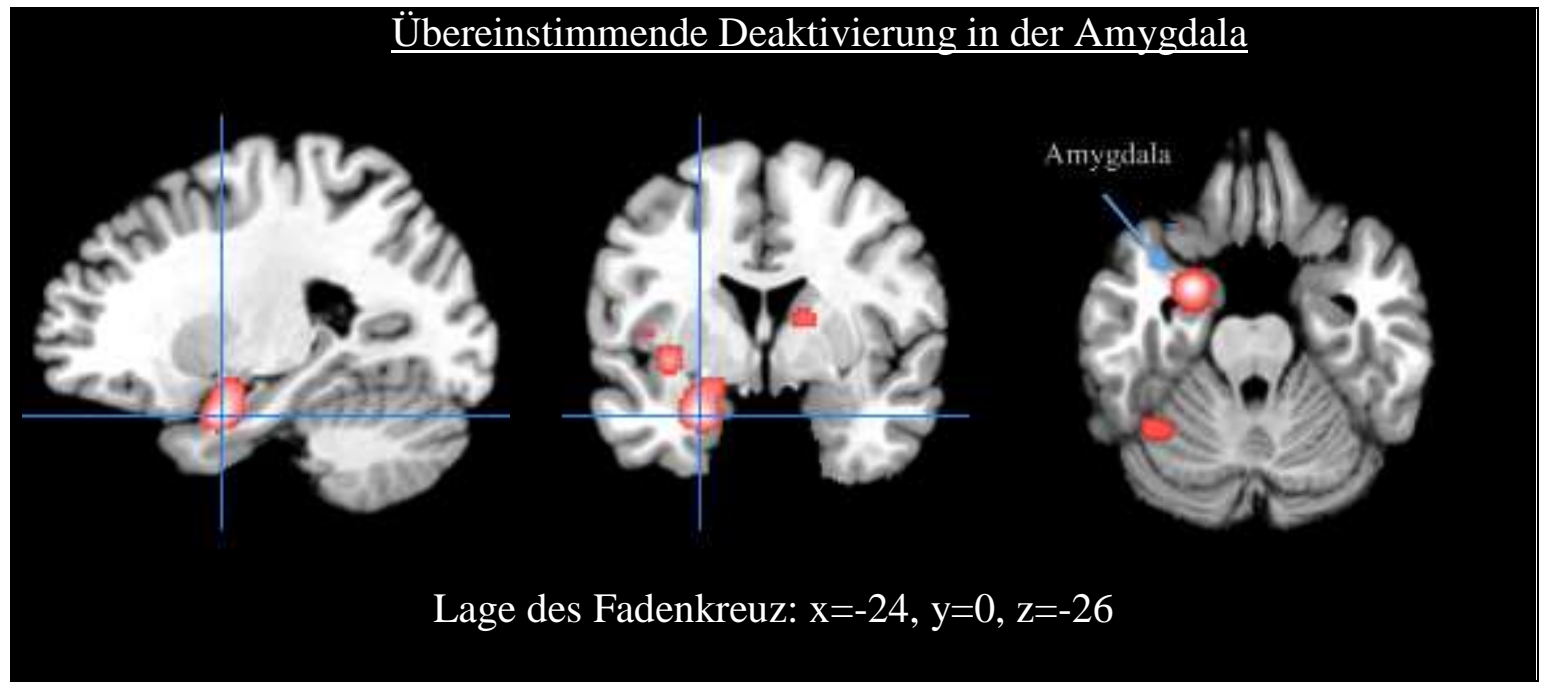




\section{Diskussion}

\subsection{Ziele und Überblick über die wichtigsten Ergebnisse dieser Arbeit}

Das Ziel dieser Arbeit war es, mit Hilfe der ALE-Metaanalyse Gehirnareale zu identifizieren, die an der Reduktion von negativen Emotionen, insbesondere der Angst, beteiligt sind. In den Studien, die in die Metaanalyse miteinbezogen wurden, erfolgte die Regulierung der negativen Emotionen entweder willentlich durch kognitive Emotionsregulation, unbewusst durch Placebobehandlung oder durch die Furchtextinktion. Die Metaanalysen basierten auf den Gehirnkoordinaten aus den Studien, die von vermehrter Aktivierung während dieses Vorgangs berichteten. Es wurde zunächst für jeden Studientyp (Emotionsregulation, Placeboeffekt, Furchtextinktion) eine Analyse durchgeführt und anschließend eine studientypübergreifende. Das Ziel war, Gehirnregionen zu identifizieren, die in allen drei Studientypen vermehrte Aktivität zeigen. Bei diesen Regionen war zu vermuten, dass sie eine Art Kontrollfunktion über wahrgenommene negative Emotionen und Angst haben.

Für eine weitere Metaanalyse wurden die Koordinaten mit verminderter Gehirnaktivität bei der Reduktion negativer Emotionen zusammengetragen. Da diese Gehirnregionen bei der Reduktion von negativen Emotionen eine Deaktivierung aufwiesen, handelte es sich folglich um Regionen, die bei der Wahrnehmung von Angst und negativen Emotionen eine Rolle spielten.

In den drei voneinander unabhängigen Metaanalysen sowie in einer studientypübergreifenden Metaanalyse über die Furchtextinktion, den Placeboeffekt und die Emotionsregulation war übereinstimmend die Region des VMPFC während der Reduktion negativer Emotionen aktiviert (Tabellen 3.1-3.3, Abbildung 3.1).

Zusätzlich deckten die Ergebnisse der Metaanalysen weitere Gehirnregionen auf, die während der Reduktion negativer Emotionen erhöhte Aktivitäten aufwiesen. Diese aktivierten Regionen waren allerdings für jeden Studientyp spezifisch und zeigten zwischen den drei Studientypen kaum Überlappungen (Tabellen 3.1-3.3). Diese Gehirnregionen sind wahrscheinlich an spezifischen Prozessen in den drei experimentellen Studientypen beteiligt.

Weiterhin fielen noch verstärkte Aktivitäten im linken anterioren Gyrus cinguli und in der rechten anterioren Inselrinde in einer Analyse auf, die nur Studien über Placeboeffekte und willentliche Emotionsregulation betrachtete (Abbildung 3.2). 
Die Ergebnisse zeigten studientypübergreifend, dass bei der Reduktion negativer Emotionen die Aktivierung im VMPFC in 64\% von einer verminderten Aktivität in der linken Amygdala begleitet wurde (Tabelle 3.5).

Insgesamt ließ sich aus den Ergebnissen schließen, dass der VMPFC wahrscheinlich ein generelles Kontrollzentrum wahrgenommener negativer Gefühle im menschlichen Gehirn darstellt, da der VMPFC in allen drei Studientypen an der Reduktion negativer Emotionen beteiligt war. Zusätzlich steht der VMPFC in einem engen funktionellen Zusammenhang mit der Amygdala, wodurch die Intensität der Angstwahrnehmung beeinflusst wird. Die Frage, ob der VMPFC im diesem Fall die Amygdala beeinflusst oder umgekehrt, kann jedoch durch diese Ergebnisse nicht abschließend beantwortet werden.

Im Verlauf dieser Diskussion werden die Funktionen des VMPFC aufgezeigt und es werden weitere mögliche Erklärungen für die beobachtete Aktivierung des VMPFC in den Studien gesucht. Anhand dieser Erklärungsmodelle basierend auf der neueren Literatur wird diskutiert, ob der VMPFC auf Grund der vorliegenden Ergebnisse als Kontrollzentrum negativer Emotionen angesehen werden kann. Weiterhin wird beleuchtet, warum manche Studien, die in die Metaanalyse miteinbezogen wurden, nicht von einer VMPFC während der Regulation negativer Emotionen berichteten.

Zusätzlich werden weitere aktivierte Gehirnregionen und ihre Funktionen betrachtet, die allerdings nur studientypspezifisch Aktivierungen aufwiesen. Es wird anhand von beschriebenen Funktionen dieser Regionen in der Literatur versucht, die beobachteten Aktivierungen in den jeweiligen Studientypen zu erklären.

Zudem werden die Ergebnisse der Paarmetaanalyse über die Placebobehandlung und die willentliche Emotionsregulation untersucht, in der insbesondere der ACC und die anteriore Inselrinde auffielen.

Anschließend wird die verminderte Aktivität der Amygdala näher beleuchtet, die in allen drei Studientypen während der Reduktion negativer Emotionen festgestellt wurde.

Abschließend werden die Grenzen dieser Metaanalysen aufgezeigt und es wird auf die Bedeutung dieser Analysen für das Verständnis von Erkrankungen wie Angststörungen eingegangen. 


\subsection{Der VMPFC: Anatomie, Einflüsse und Funktionen}

Der präfrontale Kortex ist eine Gehirnstruktur, die sich phylogenetisch wie ontogenetisch erst spät entwickelt hat. Sie ist an kognitiven und emotionalen Prozessen beteiligt und repräsentiert die höchste Ebene der funktionellen kortikalen Hierarchie (Fuster 2002).

Der VMPFC ist eine Gehirnregion, die anatomisch und funktionell aus verschiedenen Einheiten besteht (Bos et al. 2007). Er ist ein Teil des Frontalhirns und besteht aus Teilen des medialen orbitopräfrontalen Kortex und des anterioren Gyrus cingulis. Genauer setzt sich der VMPFC aus den Brodmann-Arealen 10 und 32, dem rostralen anterioren Gyrus cinguli zwischen dem medialen orbitofrontalen Kortex (BA 14) und weiteren posterioren Arealen des mediofrontalen Kortex mit den BA 8, 9 und 24 zusammen (Amodio und Frith 2006). Die Abbildung 4.1 zeigt einen Mediosagittalschnitt eines Gehirns um Abgrenzungen zwischen den präfrontalen Gehirnregionen $\mathrm{zu}$ verdeutlichen. Der VMPFC ist anatomisch und funktionell eng mit anderen Gehirnregionen verbunden - mit dem Limbischen System und speziell der Amygdala, dem Hypothalamus, dem ventralen Striatum, der anterioren Inselrinde, dem Hippokampus und dem orbitofrontalen Kortex (Simmons et al. 2006). Der VMPFC ist ein Verbindungspunkt zwischen stammesgeschichtlich neueren und differenzierteren Gehirnstrukturen, wie dem lateralen präfrontalen Regulationssystem, und phylogenetisch älteren Strukturen, wie dem emotionalen System mit Amygdala und dem intero- und exterozeptiven sensorischen Kortex (Banks et al. 2007, Delgado et al. 2008, Diekhof et al. 2011 b, Ochsner und Gross 2005, Wager et al. 2008, Wager et al. 2009 a, Wager et al. 2009 b, Watson et al. 2009). 
4. Diskussion

\section{Abbildung 4.1 Abgrenzungen zwischen den präfrontalen Regionen (Etkin et al. 2011 S. 86)}

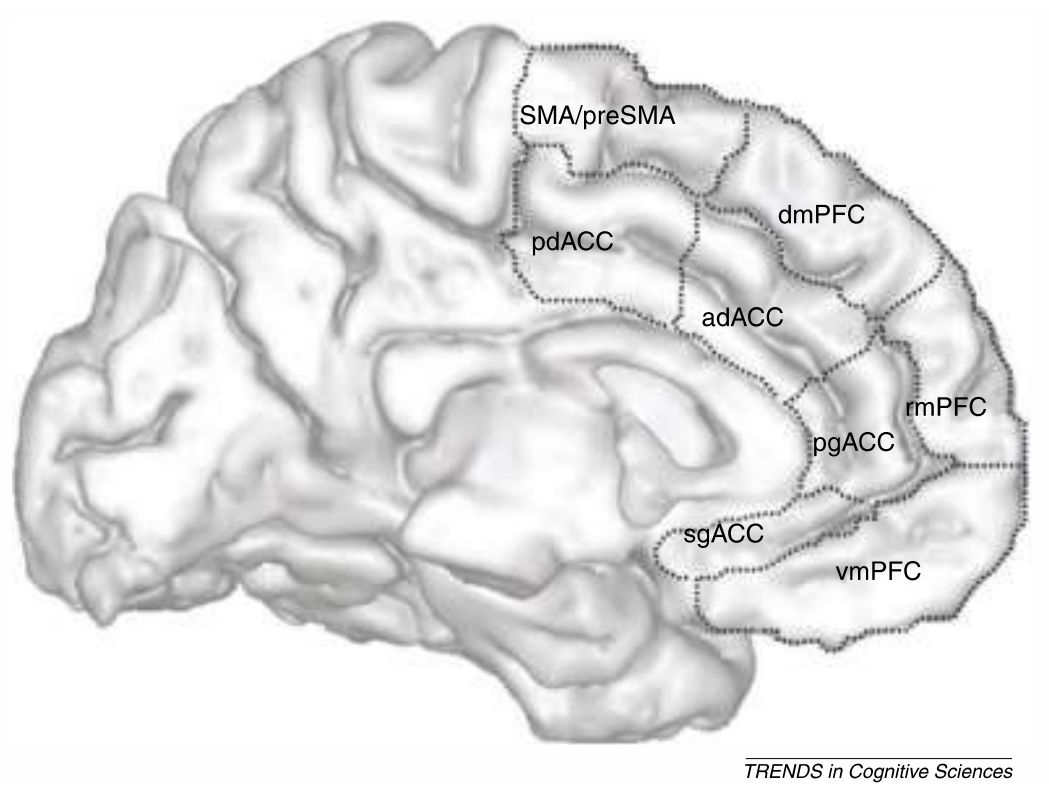

„Parcellation of ACC and mPFC subregions. Abbreviations: sg, subgenual; pg, pregenual; vm, ventromedial; rm, rostromedial; dm, dorsomedial; ad, anterior dorsal; pd, posterior dorsal.“ (Etkin et al. 2011, S. 86)

Studien haben gezeigt, dass der VMPFC verschiedene Funktionen besitzt. Einige Funktionen des VMPFC werden im Folgenden aufgeführt. Andere Funktionen der Gehirnregion können als mögliche Erklärung für die in der Metaanalyse beobachtete VMPFC-Aktivität dienen, und werden in einem folgenden Kapitel (4.2.2) näher beleuchtet.

Dem VMPFC wird u.a. eine wichtige Funktion bei emotionalen Bewertungen von Informationen und Reizen zugeschrieben (Aouizerate et al. 2007, Simmons et al. 2006, D’Argembeau et al. 2007). Dies ist die Grundlage für das Treffen von emotionalen Entscheidungen, woran der VMPFC ebenfalls beteiligt ist (Clark et al. 2008). Die Fähigkeit der emotionalen Bewertung als Voraussetzung für eine erfolgreiche Gefühlsregulation unterstützt die Annahme über die wichtige Funktion des VMPFC bei der Regulation negativer Emotionen. „Ebenso zeigt der VMPFC eine Aktivität bei der affektiven Beurteilung wie der Bearbeitung von Belohnungen und bei der sozialen Kognition wie bei der Vorstellung des Gedankenguts anderer Menschen“(Bos et al. 2007, S. 337). 
Der rACC scheint auch bei der Lösung von emotionalen Konflikten beteiligt zu sein. In einer Studie von Etkin et al. (2006) zeigte sich eine rACC-Aktivität begleitet von einer AmygdalaDeaktivierung. Auch in dem Design der Emotionsregulationsstudien der eigenen Metaanalyse zeichnete sich ein Gefühlskonflikt ab. Die Aufgabe der Probanden war es, ihre starken natürlichen Gefühle nicht zuzulassen und die ihnen dargebotene negative Situation neu zu bewerten.

Weiterhin wurde eine VMPFC-Aktivierung bei der Empfindung von Mitgefühl und Freundschaft (Güroglu et al. 2008) und bei der Identifikation mit anderen Menschen nachgewiesen (Mitchell et al. 2006). In den Emotionsstudien der eigenen Metaanalyse sollten sich die Probanden nicht in die ihnen präsentierte Situation, mit Bildern von Menschen in angsteinflößenden Situationen, hineinversetzten. Güroglus et al. (2008) und Mitchells et al. (2006) Studien zeigten, dass der VMPFC dabei hilft, Situationen zu bewerten und einzuschätzen um Emotionen regulieren zu können oder diese zu intensivieren, um Emotionen wie Empathie empfinden zu können. Zudem fanden Modinos et al. (2010) eine positive Korrelation zwischen der dispositionellen Aufmerksamkeit und dem Erfolg bei der willentlichen Emotionsregulation - Menschen, die sich in einem Zustand erhöhter Aufmerksamkeit befanden, berichteten von einem stärkeren willentlichen Regulationserfolg.

Es wird angenommen, dass die VMPFC-Aktivität auch einen Einfluss auf die Kortisolsekretion hat. Es wurde beobachtet, dass Menschen, die während der willentlichen Emotionsregulation und Neubewertung von negativen Emotionen eine verstärkte Aktivität im VMPFC und verminderte Aktivität im Bereich der Amygdala zeigten, ebenfalls eine steilere und anpassungsfähigere tägliche Kortisolausschüttung aufwiesen (Urry et al. 2006, Johnstone et al. 2007), was wichtig für ein gutes Wohlbefinden ist. Das stimmt auch mit den Erkenntnissen über Patienten mit Depressionen oder Posttraumatischen Belastungsstörungen überein, bei denen eine Fehlregulation der Kortisolsekretion gezeigt wurde (Urry et al. 2006). Ein gut adaptiertes Vermögen, auf stressige Erfahrungen zu reagieren, das gleichzeitig mit einer vermehrten Kortisolausschüttung verbunden ist, erleichtert auch die Fähigkeit, negative Gefühle zu regulieren (Urry et al. 2006). Akirav und Maroun (2007) vermuten, dass Stress das Erlernen der Furchtextinktion negativ beeinflusst. Grundlage für diese Vermutung ist, dass das Löschgedächtnis labiler als das Angstgedächtnis ist und negative Stresssituationen an Angstsituationen erinnern und so das Angstgedächtnis stabilisiert wird. 


\subsubsection{Studientypübergreifende VMPFC-Aktivität während der Regulation negativer Emotionen}

Die Auswertungen der vorliegenden Metaanalyse zeigten, dass 17 der 45 Studien aus den drei Studienbereichen eine Gehirnaktivierung im Bereich des VMPFC mit Anteilen des medialen OFC, des subgenualen und rostralen ACC und des anterioren medialen PFC aufzeigten.

Die Beobachtung von übereinstimmenden Aktivierungen im VMPFC während erfolgreicher Reduktion negativer Emotionen stimmt mit zuvor veröffentlichten Theorien überein. Diese zeigten die Funktion dieser Gehirnregion während der Reduktion negativer Emotionen auf (Delgado et al. 2008, Milad et al. 2007, Quirk und Beer 2006, Quirk et al. 2006).

Auch mit Tierstudien ergaben sich Gemeinsamkeiten. Die Ergebnisse der Tierstudien zeigten die wichtige Funktion des medialen präfrontalen Kortex bei der Regulation von Angst bei gleichzeitiger Hemmung der Amygdala (Milad und Quirk 2002, Maren und Quirk 2004, SotresBayon et al. 2004, Milad et al. 2006) und lassen somit vermuten, dass speziesübergreifend der Prozess der Furchtextinktion auf ähnliche Weise funktioniert. Tier- und Menschenstudien wiesen zudem nach, dass Schäden im frontalen Gehirnbezirk oder in der Amygdala zur Fehlregulation von Emotionen führen (Williams und Mateer 1992, Izquierdo und Murray 2005, Sanchez-Navarro et al. 2005). Ebenso bewiesen Tierstudien, in denen der präfrontale Kortex elektrisch stimuliert wurde, den hemmenden Einfluss des medialen präfrontalen Kortex auf die Amygdala (Herwig et al. 2007, Quirk et al. 2003).

Es wurde gezeigt, dass sich die Furchtextinktion aus einer kurzfristigen und längerfristigen Verarbeitung im Gedächtnis zusammensetzt (Santini et al. 2001). Es wird vermutet, dass die kurzfristige Löschverarbeitung durch einen Amygdala-abhängigen Kreislauf reguliert wird und dass die längerfristige Löschung durch den VMPFC geregelt wird, der wiederum Einfluss auf den Amygdala-abhängigen Kreislauf hat (Milad und Quirk 2002, Quirk et al. 2000, Santini et al. 2001). Das spiegelt auch die Funktion des VMPFC auf höherer Ebene wider, der die langfristige Kontrolle über die Angstwahrnehmung ausübt und bestätigt die Vermutung über die Regulationsfunktion über negative Emotionen. Genauer beeinflusst und kontrolliert der VMPFC die Amygdala während der Furchtextinktion, indem er gabanerge Zellen der Amygdala aktiviert (Milad et al. 2007, Berretta et al. 2005, Quirk et al. 2003, Rosenkranz et al. 2003) die Amygdala zur opioidvermittelten endogenen Antinozizeption anregt (Fanselow 1994). Das heißt, dass der VMPFC subkortikale 
antinozizeptive Netzwerke rekrutiert, um Kognition (Erwartung von Schmerzfreiheit) mit endogener Schmerzkontrolle/Antinozizeption zu verbinden (Bingel et al. 2006). Der RACC, als Teil des VMPFC, hat eine der höchsten Konzentrationen von Opioidrezeptoren im Gehirn (Willoch et al. 1999). Damit spielt der ACC eine zentrale Rolle bei der Schmerzwahrnehmung und deren Regulierung (Büchel et al. 2002, Petrovic und Ingvar 2002, Mohr et al. 2005), bei der emotionalen Wahrnehmung und deren Regulierung (Drevets 2000, Davidson et al. 2002) und ihrer Interaktionen (Vogt 2005).

Die Ergebnisse dieser Metaanalyse lassen vermuten, dass der VMPFC allgemeine negative emotionale Reaktionen kontrolliert, insbesondere wahrgenommene Angst.

Die zentrale Rolle des VMPFC bei der Reduktion der negativen Gefühle, ohne Betrachtung der studientypspezifischen Unterschiede, ist zum Ersten die Aktivierung während der Regulation negativer Emotionen, studientypunabhängig und auch unabhängig vom verwendeten Reiz (Schmerz oder Bilder), zum Zweiten die Herabregulation der Aktivität der Amygdala, was studientypübergreifend in $64 \%$ der Studien festzustellen war. Zusätzlich zeigte sich, dass die restlichen Gehirnregionen mit verstärkter Aktivität eher studientypspezifisch als studientypübergreifend waren und wahrscheinlich nicht im direkten funktionellen Zusammenhang mit dem VMPFC und seiner Kontrollfunktion über die Regulation negativer Emotionen standen. Es ist zu vermuten, dass diese weiteren Gehirnregionen einen anderen Einfluss auf Gefühlsprozesse haben. Die Funktionen der zusätzlich aktivierten Gehirnregionen werden im Verlauf der Diskussion ebenfalls teilweise beleuchtet.

\subsubsection{Weitere Erklärungsmodelle für die VMPFC-Aktivierung}

Es bleibt festzustellen, dass eine studienübergreifende Aktivität im VMPFC während der Regulation negativer Emotionen beobachtet werden konnte. Es stellte sich die Frage, ob der VMPFC die Funktion eines Emotions- und Angstregulationszentrums übernimmt oder ob die beobachtete VMPFC-Aktivität auf andere Weise erklärt werden kann. Der folgende Abschnitt liefert basierend auf der derzeitigen Studienlage zu den Funktionen des VMPFC mögliche 
Erklärungsmodelle für die in dieser Metaanalyse beobachtete VMPFC-Aktivität. Es wird diskutiert ob die Kontrollfunktion des VMPFC über negativen Emotionen wahrscheinlich ist.

\subsubsection{Veränderte Wahrnehmung durch Erwartungsmanipulation}

Eine Reihe von bildgebenden Studien haben angedeutet, dass die Aktivität des VMPFC von Vorurteilen und Voreingenommenheiten beeinflusst werden kann und objektive sensorische Eingänge dadurch überschrieben werden können (Diekhof et al. 2011b, Grabenhorst et al. 2008). Die Forschung zeigte, dass der VMPFC zu einem Gehirnsystem gehört, das sich mit Umweltreizen beschäftigt und diese im Gehirn eintreffenden sensorischen Informationen mit inneren Vorstellungen, die sich vom Gedächtnis, von mentalen Bildern und Selbstbeobachtung ableiten, verknüpft (Schacter et al. 2007, Summerfield et al. 2009). Reize wie Schmerz und dadurch erzeugte Gefühle werden als Zusammensetzung aus Erfahrungen angesehen, die sich wiederum aus voraussagenden Informationen und somit erlernten Erwartungen und aktuell eintreffenden Reizen aus der inneren und äußeren Welt zusammensetzen (Petrovic et al. 2005, S. 965). Viele Studien, die in diese Metaanalyse miteibezogen wurden, zeigten, dass falsche Erwartungen die Veränderung von Beurteilungen, Erfahrungen, Emotionen und neuronalen Verarbeitungsprozessen bewirken können (z. B. Diekhof et al. 2011b, Wager et al. 2004, Petrovic et al. 2005); das heißt, dass sie auch die Wahrnehmung von aversiven Stimuli verändern können. Diese während der Zeit der Erwartung wirkenden präfrontalen Einflüsse könnten auf tieferer Ebene die sensorischen und emotionalen Reaktionen regulieren (Diekhof et al. 2011b, S. 1704).

Dieses Phänomen der Erwartungsmanipulation zeigte sich bei einigen Emotionsregulations-, Placebo- und Furchtextinktionsstudien dieser Metaanalyse.

In einigen Emotionsregulationsstudien, die von einer verstärkten VMPFC-Aktivität während der Angstreduktion berichteten (z. B. Delgado et al. 2008, Johnstone et al. 2007, Urry et al. 2006), war $\mathrm{zu}$ beobachten, dass die Probanden vor Darbietung des aversiven Reizes geistige Vortäuschungsstrategien benutzten, um die negativen Gefühle zu reduzieren. Erreicht wurde dies durch Förderung beruhigender Gedanken zur Natur oder durch Umdeutung der dargebotenen Situation als unwirklich (,,imagery“ (Delgado et al. 2008, S. 836) oder „simulation“ (Johnstone et al. 2007, S. 8879, Urry et al. 2006, S. 4417)). Ebenso erfuhren die Probanden einiger Placebostudien vor Darbietung des aversiven Reizes eine Konditionierung der Placeboanalgesie (siehe Einleitung) und dadurch die Erwartung der Schmerzreduktion. Auch bei den 
Furchtextinktionsstudien lernten die Probanden eine neue Verbindung zwischen dem konditionierten Stimulus (CS) und der Abwesenheit des unkonditionierten Stimulus (UCS), bevor die Probanden wieder während des Wiederabrufs des Löschens getestet wurden (z.B. Kalisch et al. 2006b).

Generell beeinflusst die Anzahl der Versuche des Konditionierens (siehe Einleitung) vor einer Placebobehandlung die Beständigkeit des anschließenden Placeboeffekts, was vermutlich die mit dem Lernen verbundene Verstärkung der CS-UCS-Verbindung widerspiegelt (Colloca et al. 2010). Auch Petrovic et al. (2005, S. 966) sagen, dass der Placeboeffekt nur als „subjektive Welterfahrung“ beschrieben werden kann, mit einer Erwartungshaltung, die von vorausgegangenem Lernen beeinflusst worden ist. Somit ist zu vermuten, dass die VMPFCAktivität auch von dem vorherigen Lernerfolg abhängt.

Schließlich ist zu vermuten, dass der VMPFC während der Erwartung agieren könnte um somit das Wahrnehmungssystem in Richtung einer bestimmten Empfindung oder Fehlwahrnehmung zu beeinflussen (Sarinopoulos et al. 2006).

Die Studie von Plassmann et al. (2008) bestätigte die Vermutung, dass eine Erwartung die Wahrnehmung beeinflussen kann. Hier wurde vermehrte Aktivität im medialen orbitofrontalen Kortex gefunden, als die Probanden einen Wein als geschmackvoller bewerteten, nachdem sie einen Hinweis bekommen hatten, einen teuren Wein zu trinken. Bei der Verkostung des gleichen Weins mit der vorausgehenden Information, einen preiswerten Wein zu trinken, zeigte sich diese neuronale Aktivierung nicht. Ebenso wurde bei Grabenhorst et al. (2008) ein geschmacklicher Reiz durch vorhergehende Worthinweise, wie zum einen „Mononatriumglutamat“ und zum anderen „lecker und schmackhaft“, verändert. Menschliche durch Umweltreize hervorgerufene Gefühlsreaktionen sind nicht nur abhängig von Sinneseigenschaften, sondern auch von kognitiven und sprachlichen Verarbeitungen auf höherer Ebene (Grabenhorst et al. 2008). Es wurde ferner gezeigt, dass allein die Erwartung eines bevorstehenden Ereignisses die Kraft hat, grobe und unverarbeitete Formen der mentalen Stimulation auszulösen, die das wahrzunehmende erwartete Ereignis nachahmen (Holland 1990, Rescorla 1988, Rilling und Neiworth 1987), und das damit verbundene sensorische und emotionale Wahrnehmungssystem vorzeitig aktivieren (Bermpohl et al. 2006, Boly et al. 2007, Carlsson et al. 2000, Nitschke et al. 2006, Onoda et al. 2008, Ploghaus et al. 1999, Ploner et al. 2010, Porro et al. 2002). 
Der VMPFC könnte jedoch auch bei der Bewertung des dargebotenen Reizes mitwirken und somit eher in der Wahrnehmungsphase eine Rolle spielen (Grabenhorst et al. 2008).

Folglich ist zu vermuten, dass Teile des VMPFC während der Erwartung und andere während der Wahrnehmung eine Rolle spielen (Diekhof et al. 2011a).

\subsubsection{VMPFC-Aktivität bei positiven Emotionen}

Grabenhorst et al. (2008) vermuteten, dass eine Aktivität im VMPFC durch die positive Geschmacksempfindung und nicht durch die Intensität eines Geschmacksreizes erzeugt wird.

Ebenso zeigten positive Gefühle, die zur Regulation und Reduktion von negativen Emotionen dienen können, assoziierte Aktivierungen im sgACC, VMPFC und pgACC (Etkin et al. 2011) und auch Vogt et al. (2005) meinen, dass eine Aktivität im rACC mit positiven Gefühlszuständen verbunden ist.

Diese Annahme, dass der VMPFC verstärkte Aktivität in angenehmen und freundlichen Situationen zeigt, passt auch zu den Ergebnissen der eigenen Metaanalyse, da eine vermehrte VMPFC-Aktivität bei der Änderung von etwas „Negativem“ zu etwas „Positivem“ beobachtet wurde. Gleichzeitig schließt diese Funktion des VMPFC nicht die Vermutungen aus, zusätzlich eine zentrale Funktion bei der Regulation negativer Emotionen zu haben.

De Araujo et al. (2005) kamen zu ähnlichen Erkenntnissen. Sie berichteten von Aktivierungen im Bereich des VMPFC bei der Präsentation eines Geruchsreizes, der mit dem Hinweis kombiniert wurde, es handle sich um Käse anstatt um Körperschweiß. Es zeigte sich bei dem positiv besetzten ,Geruchserlebnis' eine VMPFC-Aktivität. Weiterhin berichteten McClure et al. (2004) von einer VMPFC-Aktivierung bei Versuchsteilnehmern, die ihre Lieblingsgetränke (Coca-Cola oder Pepsi) testeten. Freude und Annehmlichkeit ließen sich wieder im VMPFC erkennen. Die Freude und Vorliebe für das Getränk zeigte sich auch stärker, wenn die Probanden aus mit Getränkenamen gekennzeichneten Gläsern (z. B. Coca-Cola) statt aus namenlosen oder anonymen Gläsern tranken. Auch hier wurde die VMPFC-Aktivität von der Voreingenommenheit wie der Annahme, das Getränk sei Coca-Cola und keine Pepsi, angetrieben, womit sich der „objektive Sinneseindruck“ veränderte. Auch in dem Design der Studien, die in dieser Metaanalyse genauer betrachtet wurden, zeigte sich, dass bei den Probanden durch Hinweise eine positive Erwartung bezüglich des Reizes 
erzeugt werden konnte und die negativen Emotionen schließlich durch Regulationsformen reduziert wurden (siehe auch Abschnitt 4.2.2.1).

\subsubsection{VMPFC-Aktivität in Belohnungs- und Entscheidungssituationen}

Grabenhorst und Rolls (2011) zeigten, dass der OFC und der VMPFC wichtig sind, um Erwartungen, Belohnungen und erfahrenes Vergnügen einzuschätzen. Unterschiedliche Erwartungen in Bezug auf Belohnungen und Ergebnisse beeinflussen menschliche Gefühle und bei der Darbietung bestimmter Reize auch die emotionale Wahrnehmung (Mellers 2000).

Bei den inkludierten Studien dieser Metaanalyse ist ein wesentliches Merkmal, dass der Mensch mit einer Furcht- bzw. Schmerzreduktion belohnt wird. Dies zeigt sich beispielsweise beim Konditionieren des Furchtextinktionsgedächtnisses sowie beim konditionierten Placeboerfolg.

Auch Petrovic et al. (2005) erwähnten Ähnlichkeiten zwischen Placeboanalgesie und Belohnungsverarbeitung, denn bei beiden spielt die Vorahnung eines positiven Ergebnisses eine Rolle und sie sind abhängig von Erwartungen. Zusätzlich verwiesen Grabenhorst et al. (2008, S. 1555) darauf, dass im VMPFC, insbesondere im orbitofrontalen Kortex und im pregenualen Gyrus cinguli, der emotionale Wert von vielen unterschiedlichen sensorischen Belohnungen repräsentiert wird wie beispielsweise von geschmacklichen, visuellen oder somatosensorischen Stimuli (Kringelbach 2005, Rolls 2007, Padoa-Schioppa und Assad 2006).

Somit könnte die in dieser Metaanalyse beobachtete VMPFC-Aktivierung auch teilweise durch die Einschaltung des Belohnungssystems erklärt werden.

Wie schon im Kapitel 4.1.1 erwähnt, ist der präfrontale Kortex wichtig, um Entscheidungen zu treffen (Grabenhorst und Rolls 2011). Bei der willentlichen Emotionsregulation wird auch die Entscheidung getroffen, eine Situation positiver zu bewerten. Dies könnte somit als ein weiteres Erklärungsmodell für die VMPFC-Beteiligung bei der Emotionsregulation dienen.

Diese Annahme wurde auch durch Bechara et al. (1994) unterstützt. Die Forscher vermuteten, dass Patienten mit Schäden im VMPFC unsensibel für zukünftige Belohnungen oder Bestrafungen sind und Probleme beim Treffen von Entscheidungen zeigen. 


\subsubsection{Fazit der Erklärungsmodelle}

Alle diese Erklärungsversuche können die in dieser Metaanalyse beobachtete VMPFC-Aktivität erklären, jedoch schließen sie nicht aus, dass der VMPFC die vermutete Funktion des Regulationszentrums über negative Emotionen besitzt. Die Modelle zur Erklärung der VMPFCAktivität lassen sich nicht auf alle in dieser Metaanalyse untersuchten Studien beziehen. Das Erklärungsmodell der VMPFC-Aktivität in Belohnungssituationen lässt sich nur in den Furchtextinktions- und Placebostudien beobachten und die Erklärung der VMPFC-Aktivität in Entscheidungssituationen kann man nur auf die willentlichen Emotionsregulationsstudien beziehen. Wie im Kapitel 4.2.2.2 beschrieben zeigte sich eine VMPFC-Aktivität bei der Empfindung von positiven Emotionen. Diese Ergebnisse bestätigen die erfolgreiche Regulation der Emotionen in den Studien der vorliegenden Metaanalyse, da in diesen eine VMPFC-Aktivität nach bzw. während der Regulation negativer Emotionen vorlag. Da die Regulation negativer Emotionen allen drei Studientypen gemein war, lässt sich vermuten, dass der VMPFC eine entscheidende und zentrale Rolle bei der Regulation negativer Emotionen spielt.

\subsubsection{Inkludierte Studien der Metaanalyse ohne VMPFC-Aktivität}

Es ist festzustellen, dass nicht alle Studien dieser Metaanalyse von einer verstärkten Aktivität im Bereich des VMPFC während der Regulation negativer Emotionen berichteten. 28 der 45 Studien, die mit in die Metaanalyse einbezogen wurden, berichteten nicht über eine verstärkte Gehirnaktivität im Bereich des VMPFC. Die Summe der 28 Studien setzte sich zusammen aus vier von neun Furchtextinktionsstudien (Knight et al. 2004, Lang et al. 2009, Spoormaker et al. 2010, Yágüez et al. 2005), aus weiteren sechs der 13 Placebostudien (Kong et al. 2009, Petrovic et al. 2002, Sarinopoulos et al. 2006, Wager et al. 2004 (1) und (2), Zubieta et al. 2005) und schließlich aus 18 der 23 Emotionsregulationsstudien (Domes et al. 2010, Drabant et al. 2008, Eippert et al. 2007, Goldin et al. 2007, Harenski und Hamann 2006, Herwig et al. 2007, Kalisch et al. 2006c, Kim und Hamann 2007, Koenigsberg et al. 2010, Kross et al. 2009, McRae et al. 2009, Modinos 2010, Ochsner et al. 2002, Ochsner et al. 2004, Wager et al. 2008, Walter et al. 2009, Winecoff et al. 2010) Grundsätzlich muss darauf hingewiesen werden, dass aus dem Studienbereich der Emotionsregulation nur eine geringe Anzahl der Studien von einer verstärkten Aktivierung im 
ventromedialen präfrontalen Gehirnbereich berichteten. Die Ursachen dieser abweichenden Ergebnisse der 28 zuvor genannten Studien werden im Folgenden näher betrachtet.

Der Grund dieser Ergebnisunterschiede könnte durch verschiedenen Faktoren erklärt werden, wie etwa unterschiedliche Reize, unterschiedliche Gefühlszustände oder Unterschiede in den Regulationsstrategien, die in den Studien benutzt wurden (Delgado et al. 2008, Ochsner et al. 2004). Zudem lagen in einigen Studien weitere kleinere Unterschiede im Studiendesign vor, die möglicherweise zu der Divergenz der Ergebnisse beigetragen haben können. Beispielsweise gaben Domes et al. (2010, S. 760) den Probanden erst nach der Präsentation der aversiven Bilder die Aufgabe zur Neubewertung (siehe auch Kross et al. (2009) und Modinos et al. (2010)). Im Gegensatz dazu wurde den Probanden in den meisten der Studien (z. B. Delgado et al. (2008)) die Aufgabe zur Neubewertung vor Darbietung des aversiven Reizes erteilt.

Außerdem können die Entwicklung, das Alter, die Anwesenheit von leichten Depressionen oder Ängsten sowie die generell individuellen und kulturellen Unterschiede der Probanden emotionale Reaktionen und somit auch das emotionale Regulationsvermögen und die Wirksamkeit von Neubewertungsstrategien beeinflussen (Davidson 2000, Mather et al. 2004). Weiterhin ist festzustellen, dass die Studien sich in der Art der Reize unterschieden. Drabant et al. (2008) beispielsweise benutzten angsterfüllte und ärgerliche Gesichter als Stimulus, Harenski und Hamann (2006) verwendeten Bilder von moralischen Übertretungen und Banks et al. (2007) gebrauchten Bilder mit angsterzeugenden und traurigen Szenen von brennenden Häusern oder Beerdigungen. Dies kann bedeuten, dass manche Reize stärkere emotionale Erregung bei den Probanden auslösten als andere und manche Stimuli eine moralische Beurteilung bei den Probanden induzierten (Bishop et al. 2004) und auf Grund der unterschiedlichen Anregung des Urteilsvermögens unterschiedliche Gehirnareale aktiviert wurden.

In den meisten Studien wurde nicht das komplette Gehirn nach verstärkter Aktivierung bei der Regulation negativer Emotionen durchsucht, sondern man beschränkte sich auf Regionen von besonderem Interesse (Regions of interests = ROIs). Diese ROIs variierten studienabhängig, was ein weiterer Grund für die Divergenz der Ergebnisse sein könnte.

Andere Ursachen für die in einigen Studien fehlende VMPFC-Aktivität können weitere Mechanismen im Gehirn sein, die negative emotionale Reaktionen regulieren; so könnte die laterale präfrontale Gehirnregion einen Einfluss haben, die eine Rolle bei Konfliktregelungen 
(Sohn et al. 2007) und bei der Hemmung von Reaktionen (Tsujimoto et al. 2007) spielt. Die Regionen, die auch eine verstärkte Aktivierung während der Reduktion von negativen Gefühlen zeigen, ergänzen wahrscheinlich die VMPFC-Mechanismen. Man könnte auch annehmen, dass geistige Regulationsstrategien, wie die willentliche Veränderung der Interpretation bestimmter Situationen, möglicherweise indirekt reflexartig mit Angst verbundene Reaktionen in der Amygdala und in den sensorischen Rindenbereichen abschwächen. Dies könnte im Gegenzug die Notwendigkeit direkter Eingriffe und Interventionen des ventromedialen präfrontalen Kortex reduzieren. Dies könnte auch als Erklärungsmodell für die in allen Studien fehlende Übereinstimmung der Gehirnaktivität im VMPFC dienen.

Die Betrachtung der Vermutungen von Ochsner und Gross (2005), die das Regulieren von Gefühlen in zwei mögliche Kontrollprozesse unterteilen, kann ebenfalls einen Hinweis für die einigen Studien fehlende VMPFC-Aktivität geben.

Zum einen existiert ein Kontrollsystem, das bei Gefühlsregulationsprozessen wie bei der Furchtextinktion verwendet wird und nur den ventralen präfrontalen und den orbitofrontalen Kortex einbezieht. Dieser schätzt den emotionalen Wert des zugehörigen Reizes ein und leitet diese Information direkt über eine reziproke Verbindung an die Amygdala weiter. Somit hat ein emotionales Bewertungssystem wie die Amygdala umgekehrt auch Einfluss auf den VMPFC. Drei der Furchtextinktionsstudien der Metaanalyse bestätigen diese Annahme, indem sie von verstärkter Aktivierung im VMPFC bei der Furchtreduktion berichteten.

Zum anderen gibt es ein Kontrollsystem, das den dorsalen präfrontalen Kortex einbezieht, der nur wenige bis keine direkten Verbindungen zur Amygdala besitzt. Dieses Kontrollsystem wird nur aus bestimmten Gründen genutzt und zeigt, wie Verbindungen zwischen Reizen und emotionalen Reaktionen geändert werden können. Dies geschieht indirekt über Verbindungen mit dem ventralen Kontrollsystem, das Verarbeitungsprozesse anregt, oder im Wahrnehmungsgedächtnis, das alternative Interpretationen der Reize vorschlägt, die wiederum die neuen Informationen direkt an das Gefühlssystem weiterleiten. Diese zweite Möglichkeit der Gefühlsregulation wird bei Neubewertungsstrategien wie der Emotionsregulation und bei der Placebobehandlung angewandt (Ochsner und Gross 2005). Die verstärkte Aktivität im DPFC zeigten auch die Ergebnisse der Metaanalyse mit den Emotionsregulationsstudien. Möglicherweise kaschierte dieses Ergebnis die 
VMPFC-Aktivität, die nicht so prägnant war und damit nicht in den Studien aufgezeigt wurde, da sie nicht die Aktivitätsschwelle erreichte, ab der Gehirnaktivierungen aufgezeichnet wurden.

Zusätzlich wurde gezeigt, dass die funktionelle Wichtigkeit einer Gehirnregion in bestimmten Situationen nicht nur von der Stärke der Aktivierung dieser Region abhängt.

Man fand heraus, dass Wiederholungen bestimmter Situationen (wie beim Konditionieren bei der Furchtextinktion und der Placebobehandlung oder wie bei der häufig benötigten Emotionsregulation) zu verstärkten Verbindungen zwischen den Gehirnregionen führt, die bei der Verarbeitung beteiligt sind. Diese fördern dann den Erfolg der Verarbeitung und verlangen reduzierte Verarbeitungsprozesse der involvierten Regionen (Ghuman et al. 2008, S. 8405). Somit kann man nicht ausschließen, dass auch in den Studien, die nicht von einer verstärkten VMPFCAktivität während der Regulation negativer Emotionen berichten, der VMPFC als Kontroll- und Regulationszentrum fungiert hat und diese Aktivitätsbeteiligung einfach nur nicht angezeigt wurde oder die Aktivität unter der Aktivitätsschwelle lag, ab der Aktivitäten in den Studien erfasst wurden.

\subsection{Weitere Gehirnaktivierungen während der Regulation negativer Emotionen}

Die Auswertungen der Metaanalyse wiesen noch weitere Gehirnregionen mit verstärkter Aktivität während der Regulation von Emotionen auf, jedoch waren keine weiteren Übereinstimmungen zwischen allen drei Studientypen zur Furchtextinktion, zum Placeboeffekt und zur Emotionsregulation erkennbar. Es zeigten sich demnach nur studientypspezifische Aktivierungen. In der Paarmetaanalyse über die Placebo- und Emotionsregulationsstudien fielen jedoch gemeinsame Gehirnaktivierungen auf. Gründe für diese Unterschiede könnten die unterschiedliche Reizmodalität in den Studientypen und die Variationen in den kognitiven Aufgaben sein. Bei den Furchtextinktionsstudien erfolgte die Gefühlsregulierung lediglich passiv, während bei den Emotionsregulationsstudien die Probanden willentlich und aktiv die dargebotene Situation neu bewerten mussten. Als Gemeinsamkeit zwischen den Emotionsregulationsstudien und den

Placebostudien war die permanente Darbietung des negativen Reizes und die geistig anspruchsvollere Form der Gefühlsregulation zu sehen. 
Die folgenden Abschnitte zeigen Funktionen einiger aktivierter Gehirnregionen auf. Es werden nur die in der Literatur beschriebenen Funktionen der Gehirnregionen angesprochen, durch die die Aktivierung der Gehirnregion in den jeweiligen Studientypen erklärt werden kann.

Weiterhin ist $\mathrm{zu}$ betonen, dass diese beobachteten Gehirnaktivierungen von untergeordneter Relevanz für das Gesamtergebnis dieser Metaanalyse sind.

\subsubsection{Weitere Gehirnaktivierungen in Emotionsregulationsstudien}

Bei der Auswertung der Ergebnisse fiel auf, dass in den Emotionsregulationsstudien der dorsolaterale PFC und der dorsomediale PFC, neben dem VMPFC, verstärkte Gehirnaktivierungen während der Regulation der negativen Emotionen aufzeigten. Der am meisten frontal gelegene Kortex wird als präfrontaler Kortex bezeichnet. Die präfrontalen Gehirnareale spielen eine Rolle bei der geistigen Kontrollverarbeitung, Zielrepräsentation und Bewertung von Gefühlsreizen auf höherer Ebene (Kalisch et al. 2006b, Miller und Cohen 2001). Diese Fähigkeiten wurden von den Probanden der Emotionsregulationsstudien verlangt. Abbildung 4.2 zeigt die anatomische Lage der lateralen und medialen präfrontalen Regionen auf.

Abbildung 4.2: links ist eine laterale sagittale Ansicht, rechts eine mediale sagittale Ansicht des menschlichen Gehirns dargestellt. Es werden die verschiedenen lateralen und medialen präfrontalen Regionen gezeigt. (Ray und Zald 2012, Anhang Abbildung 1)
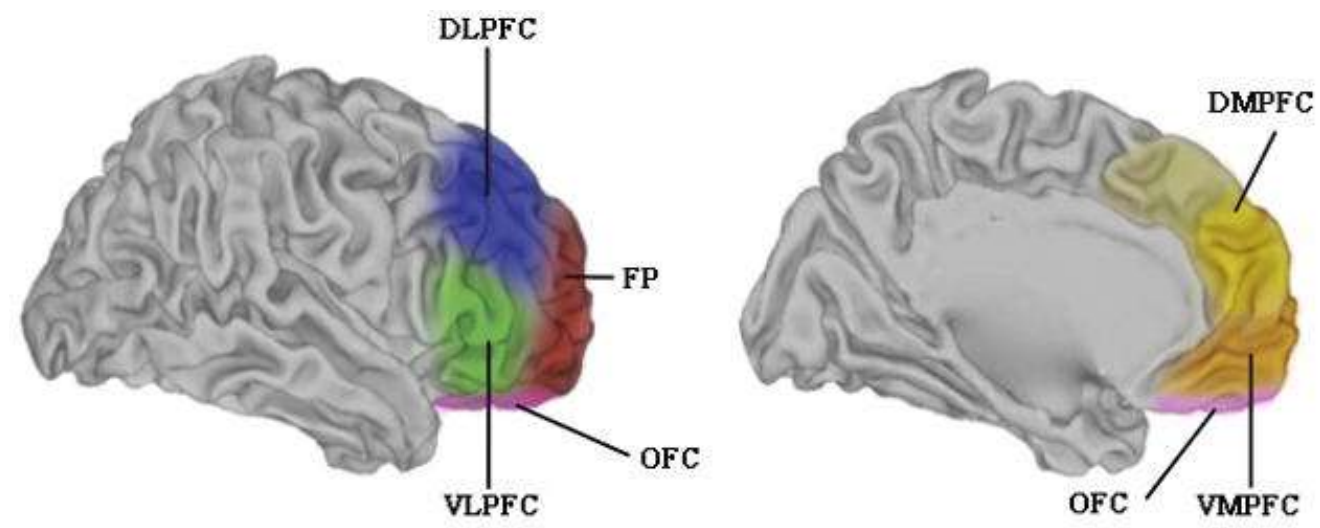


\subsubsection{LPFC}

Der LPFC ist, unabhängig vom Reiz, verantwortlich für die Überwachung von Handlungen und die kognitive Verarbeitung (Sommer et al. 2007). Grabenhorst und Rolls (2011) zeigten, dass der laterale präfrontale Kortex an der willentlichen Selbstkontrolle beteiligt ist. Das kann darauf schließen lassen, dass der LPFC generell sowohl an der willentlichen Kontrolle wie auch an der willentlichen Emotionsregulation beteiligt ist. Der LPFC zeigt eine Funktion bei der Vorbereitung auf Konflikte, bei der Regelung von Konflikten, der Problemlösung und der Anpassung an Konflikte (Sohn et al. 2007). Zusätzlich nahmen Etkin et al. (2011) bei der Konfliktbeurteilung eine Beteiligung der Amygdala, des dACC, des DMPFC und des DLPFC an. Die Probanden in den Emotionsregulationsstudien der Metaanalyse wurden auch mit einem Konflikt konfrontiert. Dieser innere Konflikt entstand bei Probanden zwischen den erzeugten ängstlichen Emotionen und der Neubewertung der dargebotenen Situation zum Positiven.

Der LPFC hat zusätzlich eine Funktion bei der Koordination zweier zeitgleicher Aufgaben, wie einer auditorischen und einer visuellen (Szameitat et al. 2006). Auch in den Emotionsregulationsstudien mussten die Probanden zwei Aufgaben gleichzeitig bewältigen. Sie wurden visuellen Reizen ausgesetzt und gleichzeitig sollten sie die Situationen neu interpretieren. Zusätzlich ist der LPFC ein Teil des Arbeitsgedächtnisses. Es ist für die Aufrechterhaltung von abrufbaren Informationen zuständig (Smith und Jonides 1999) und spielt wie schon im Kapitel 4.2.3 beschrieben eine Rolle bei der Hemmung von Reaktionen (Tsujimoto et al. 2007). Auch nach den Emotionsregulationsstudien hemmen die Probanden ihre ursprünglichen Gefühle und bewerten die dargebotene Situation neu.

\subsubsection{DLPFC}

Der DLPFC ist mit der Repräsentation und Aufrechterhaltung von Informationen assoziiert, die für die kognitive Kontrolle benötigt werden (Miller und Cohen 2001, MacDonald et al. 2000). Auch in den Emotionsregulationsstudien dieser Metaanalyse wird die kognitive Kontrolle der Probanden erwartet. Weiterhin scheint der DLPFC bei der Konzentration auf relevante Reize eine Rolle zu spielen (Orr JM und Weissmann 2009). Die Probanden mussten sich in den Emotionsregulationsstudien dieser Metaanalyse auf die ihnen gestellte Aufgabe konzentrieren und die dargebotene Situation neu bewerten. 
Es ist bekannt, dass Schäden im DLPFC zu Depressionen führen (Davidson 2002). Dies ist wahrscheinlich das Ergebnis einer verminderten Fähigkeit, positive Gefühle zu nutzen (Mineka et al. 1998). Bei den Emotionsregulationsstudien wurde ebenso eine DLPFC-Aktivität beobachtet, was möglicherweise an der Reduktion der negativen Emotionen und der damit verbundenen Bewertung der Situation zum Positiven liegt.

\subsubsection{3 dACC}

Rushworth et al. (2007) vermuteten, dass der dACC bei der Beurteilung von Aktionen und Handlungen aktiv ist, im Gegensatz zum VMPFC, der eine Funktion bei der Bewertung von Reizen hat (Schiller und Delgado 2010). Diese Annahmen stimmen auch mit den Ergebnissen der in dieser Metaanalyse untersuchten Emotionsregulationsstudien überein, die eine verstärkte dACC-Aktivität aufzeigten. In diesem Studiendesign mussten die Probanden die ihnen dargebotenen negativen Situationen neu bewerten, das heißt aktiv handeln, im Vergleich zu dem passiv konditionierenden Placeboeffekt oder der Furchtextinktion. Auch die in dieser Metaanalyse beobachtete VMPFCAktivierung kann man durch die Bewertung von Reizen durch die Probanden erklären.

Eine Aktivierung der dACC-Region wurde außerdem bei der Manipulation und Änderung von Erwartungen (Keltner et al. 2006) gezeigt. Dabei werden vorherige Gedanken, ein Verhalten oder eine ursprüngliche Einstellung geändert, was vergleichbar ist mit dem Design der Emotionsregulationsstudien.

\subsubsection{DMPFC}

Generell wird der DMPFC verbunden mit emotionalem Bewusstsein (Kalisch 2006a, Mechias et al. 2010), der Beeinflussung der Gefühlswelt (Paradiso et al. 1999, Gallagher et al. 2000, Happe et al. 1996) und deren Verarbeitung (Gusnard et al. 2001).

Verarbeitungsprozesse bezüglich der eigenen Person zeigten eine Beteiligung des VMPFC und des DMPFC auf. Die Perspektive Anderer einzunehmen, bezieht nur den DMPFC mit ein. Der DMPFC scheint somit bei der Differenzierung der eigenen Perspektive von anderen Perspektiven eine Rolle zu spielen (D’Argembeau et al. 2007). Ebenso sagten Mitchell et al. (2006), dass der DMPFC eine Beteiligung bei der Abgrenzung des Individuums zu einem anderen Menschen zeigt. Auch die Probanden in den Emotionsregulationsstudien dieser Metaanalyse sollten sich von den aversiven Bildern mit Personen in unerfreulichen Situationen distanzieren, was die verstärkte DMPFC- 
Aktivität in den Studien erklären könnte.

\subsubsection{Parietaler Kortex, temporaler Kortex, inferiorer frontaler Gyrus}

Zusätzlich war die negative Gefühlsregulation begleitet von verstärkten Aktivierungen in den Bereichen des parietalen und des temporalen Kortex, die auch bei der visuellen Aufmerksamkeit eine Rolle spielen (Corbetta und Shulman 2002, Corbetta et al. 2008, Gruber et al. 2009). Diese Aktivität kann durch die visuelle Reizform in den Emotionsregulationsstudien erklärt werden. Eine weitere Rolle des temporalen Kortex zusammen mit dem Parahippocampus spielt die Speicherung und der Wiederabruf von Erinnerungen, um das Verhalten und Entscheidungen zu leiten (Bar 2007, Bar 2009, Bar et al. 2008, Mobbs et al. 2006, Olson et al. 2007). Auch in den Emotionsregulationsstudien sollten die Probanden sich an etwas Positives oder an Beruhigendes aus der Natur erinnern.

Der parietale Kortex zeigt zusätzlich eine Beteiligung bei der Erkennung von aversiven Reizen, auch wenn sie anderen Personen zugefügt werden (Benuzzi et al. 2008). Auch den Probanden der Emotionsregulationsstudien wurden aversive Situationen präsentiert, was die Beteiligung des parietalen Kortex erklären könnte. Außerdem ist anzunehmen, dass der parietale Kortex bei der willentlichen Umlenkung der Aufmerksamkeit von gefühlsbetonten negativen Reizen auf gefühlsneutrale Aspekte involviert ist und so eine Neubewertung der Situation auf höherer Ebene entsteht (Diekhof et al. 2011a).

Weiterhin zeigte der inferiore frontale Gyrus Gehirnaktivität während der willentlichen Regulation negativer Emotionen. Dieser ist beim Treffen von Entscheidungen wichtig und zeigt eine Beteiligung bei der Hemmung von Reaktionen (Brown et al. 2012). Die Probanden in den Emotionsregulationsstudien dieser Metaanalyse entschieden sich, die ihnen präsentierten Bilder zum Positiven zu bewerten und hemmten folglich ihre physiologische Angstreaktion.

\subsubsection{Weitere Gehirnaktivierungen in Placebostudien}

In den Studien über den Placeboeffekt zeigten unter anderem der anteriore OFC und der laterale inferiore frontale Kortex während der Reduktion negativer Gefühle eine verstärkte Gehirnaktivität. Diese verstärkte Aktivierung repräsentiert verschiedene Aspekte der 
Placebostudien, wie beispielsweise die „Placeboerwartung“, die weitere regulierende Gehirnareale während der Zeit der Erwartung mit einbeziehen (Petrovic et al. 2005, Sarinopoulos et al. 2006, Wager et al. 2004, Atlas et al. 2010). Zusätzlich könnte man vermuten, dass diese Gehirnregionen den placeboinduzierten Effekt der opioiden Neurotransmission widerspiegeln (Scott et al. 2008, Zubieta at al. 2005).

\subsubsection{OFC}

Eine Schlüsselfunktion des OFC ist die Überwachung und Regulierung der externen Reize (Gottfried et al. 2003, Kringelbach und Rolls 2003, O’Doherty et al. 2001, Tremblay und Schultz 1999), um ein zielgerichtetes Verhalten durchzuführen (Schoenbaum und Setlow 2001). Ebenso zeigt der OFC eine Beteiligung bei der emotionalen Beurteilung von Informationen unter Einbeziehung von Vorwissen (Aouizerate et al. 2007). Auch in den Placebostudien wurden die Probanden Schmerzreizen ausgesetzt, die sie bewerten sollten mit dem Vorwissen, eine Pille mit analgetischer Wirkung erhalten zu haben. Eine OFC-Aktivität wurde auch bei der Verbesserung des wahrgenommenen und zugeführten Schmerzes beobachtet (Wrase et al. 2007). Dies zeigte sich auch in den Placebostudien - die Probanden empfanden nach der Placebobehandlung eine Schmerzreduktion. Zusätzlich ist der OFC beim Konditionieren beteiligt (Gottfried und Dolan 2004). In den Placebostudien erlebten die Probanden eine Form der Konditionierung, indem sie lernten, dass eine Pille einen analgetischen Effekt hat. Weiterhin zeigt der OFC eine Funktion beim Abruf des emotionalen Gedächtnisses (Hakamata et al. 2007). Durch die Konditionierung kann das zuvor Erlernte wieder abgerufen werden. Die Probanden der Placebostudien erinnerten sich an die analgetische Wirkung von Pillen.

\subsubsection{IPFC}

Wie bereits beschrieben, spielt der inferiore PFC unter anderem beim Treffen von Entscheidungen eine Rolle und ist beteiligt an der Hemmung von Reaktionen (Brown et al. 2012). Auch die Placebostudien zeigten, dass das ursprüngliche Angstgedächtnis gehemmt und das konditionierte Gedächtnis mit der Information über den analgetischen Effekt einer Pille abgerufen wird. 


\subsubsection{Weitere Gehirnaktivierungen in Furchtextinktionsstudien}

Die Metaanalyse der Studien über die Furchtextinktion ergab neben dem VMPFC weitere Gehirnregionen, die während der Reduktion negativer Gefühle verstärkte Aktivität aufweisen. Darunter waren neben dem sgACC und dem medialen Gyrus cinguli der linke Parahippocampus und die rechte Uvula vermis des Cerebellums. Der sgACC und der mediale ACC liegen dem VMPFC anatomisch nah an. Die neuere Literatur bestätigt die wichtige Funktion des sgACC bei der Furchtextinktion (z. B. Etkin et al. 2011).

Wie bereits erwähnt, ist der Parahippocampus an der Speicherung und dem Wiederabruf von Erinnerungen beteiligt, was zur Anpassung von Verhalten und Entscheidungen führt (Bar 2007, Bar 2009, Bar et al. 2008, Mobbs et al. 2006, Olson et al. 2007).

Dem Vermis cerbelli als Teil des Spinocerebellums werden u.a. Einfluss auf Affekt und Verhalten zugeschrieben (Schmahmann und Caplan 2006). Diese Funktion könnte die Gehirnaktivierung während der Furchtextinktion erklären.

\subsubsection{Paarmetaanalyse von Emotionsregulations- und Placebostudien}

Die Paarmetaanalyse von den Placebo- und Emotionsregulationsstudien zeigte zwei weitere übereinstimmende Gehirnareale mit verstärkter Aktivität während der Regulation der negativen Emotionen - zum einen der linke ACC und zum anderen die rechte anteriore Inselrinde. Grundlage dieser Metaanalyse waren Gemeinsamkeiten zwischen den beiden Studientypen. Eine dieser Gemeinsamkeiten ist die geistig anspruchsvollere Regulation der negativen Emotionen durch den Placeboeffekt und durch die willentliche Emotionsregulation bzw. durch die Neubewertung im Gegensatz zur Furchtextinktion, welche auch von Tieren erlernbar ist. Das bewusste Wahrnehmen von Gefühlen hat die Emotionsregulation mit dem Placeboeffekt gemein. Bei den Emotionsregulationsstudien nahmen die Patienten die negativen Situationen, die ihnen präsentiert wurden, bewusst wahr, bevor sie ihren Inhalt neu bewerteten, und auch bei den Placebostudien war den Patienten bewusst, dass ihnen ein Schmerzreiz zugefügt wird. Dieses Bewusstsein zeigte sich im Vergleich dazu nicht bei den Furchtextinktionsstudien, was eine weitere mögliche Erklärung für das übereinstimmende Ergebnis der Paaranalyse von Placebo- und Emotionsregulationsstudien ist. Eine weitere Übereinstimmung ist die Präsenz des negativen Reizes während der 
Gefühlsregulation in den Emotionsregulationsstudien und den Placebostudien. Im Vergleich dazu wurde in den Furchtextinktionsstudien der konditionierte Reiz (CS) ohne den negativen und aversiven unkonditionierten Reiz (UCS) dargeboten. Man könnte demnach annehmen, dass die Präsenz des negativen Reizes während der Regulation einen größeren Regulationsaufwand erfordert, um mit der Situation in gewünschter Weise umzugehen. Dies passt auch zu früheren Erkenntnissen, die besagten, dass der ACC anders als bei der automatischen Herabregulation von negativen Emotionen eine Rolle bei der bemühten und mit Anstrengung verbundenen Emotionsund Angstregulation spielt (Eippert et al. 2007, Kim et al. 2007, Modinos et al. 2010).

Diese Gemeinsamkeiten von Placebostudien und Emotionsregulationsstudien scheinen ursächlich für die gemeinsamen Aktivierungspunkte im Gehirn während der Regulation negativer Emotionen zu sein.

Die folgenden Abschnitte zeigen einige Funktionen der aktivierten Gehirnregionen auf. Es werden nur die Funktionen der Gehirnregionen angesprochen, durch die die Aktivierung der entsprechenden Gehirnregion in den Placebo- und Emotionsregulationsstudien erklärt werden kann.

\subsubsection{ACC}

Der ACC besteht aus einem ventralen Anteil, einer gefühlsbetonten Region und einem kognitiven Anteil der dorsalen ACC-Region. Der ventrale Bereich ist mit dem emotionalen und motivationalen Status und der Regulierung autonomer Reaktionen assoziiert. Der dorsale Bereich des ACC beinhaltet viele Funktionen wie die Aufmerksamkeit, das Arbeitsgedächtnis, Fehlererkennung, Überwachung von Konflikten und die Reaktionsauswahl (Aouizerate et al. 2007).

Der rostrale ACC als Teil des VMPFC zeigte eine Beteiligung bei der Erwartung eines positiven Ereignisses (Sharot et al. 2007). Dies passt zu dem Studiendesign der Emotionsregulations- und Placebostudien. Bei den Probanden wurde die negative Erwartung reduziert. In den Emotionsregulationsstudien wurde den Probanden die Aufgabe erteilt, eine präsentierte Situation distanziert zu betrachten. In den Placebostudien wurden die Probanden einer Placebobehandlung unterzogen und folglich die Erwartung, Schmerz zu erfahren, reduziert. 
Botvinick (2007) fand, dass der ACC wichtig für die Überwachung von Verarbeitungsprozessen und die Einschätzung über die Notwendigkeit kognitiver Kontrolle ist. Es ist anzunehmen, dass eine erfolgreiche Regulation negativer Emotionen abhängig ist von dem Gebrauch dieses Prozesses.

Weiterhin ist der ACC wichtig für die Konzentration und erfüllt die Aufgabe, Ablenkungen zu minimieren (Orr JM und Weissmann 2009). Die kognitiv anspruchsvolleren Formen, durch willentliche Emotionsregulation und die Placebobehandlung negative Emotionen zu regulieren, verlangten die Konzentration der Probanden.

Der ACC ist auch an der Wahrnehmung von Schmerz beteiligt und für die Auf- und Abregulierung der Aktivität jener Gehirnregionen zuständig, die wie die Amygdala und der PAG opioidregulierte antinozizeptive Antworten hervorrufen (Eippert et al. 2009, Petrovic et al. 2002). Diese beobachtete Funktion des ACC könnte auch die ACC-Aktivierung in den Placebostudien erklären. Außerdem zeigte sich eine ACC-Beteiligung bei der Bewertung von Situationen (Lamm et al. 2010) wie bei der willentlichen Neubewertung von Emotionen.

Zusätzlich spielt der ACC eine Rolle bei der Selbstregulierung (Posner et al. 2007), beim Treffen von Entscheidungen (Walton und Mars 2007) und ist ein Teil des Belohnungssystems (Hauber und Sommer 2009). Auch diese Funktionen wurden von den Probanden der Studien dieser Metaanalyse verlangt.

\subsubsection{Anteriore Inselrinde}

Die anteriore Inselrinde ist beteiligt an der Reizverarbeitung (Corbetta und Shulman 2002) sowie an der interozeptiven Wahrnehmung (Craig 2009, Critchley et al. 2004).

Benuzzi et al. (2008) zeigten eine Aktivierung der Inselrinde bei der Schmerzwahrnehmung und beim Betrachten von negativen und abstoßenden Videos. Freund et al. (2007) differenzierten genauer den anterioren Bereich der Inselrinde, der für die Schmerzwahrnehmung verantwortlich ist, vom posterioren Bereich, der eine Rolle bei der Schmerzunterdrückung spielt. Diese Beobachtung wird durch das Ergebnis der Paaranalyse von Emotionsregulationsstudien und Placebostudien bestätigt. In den Emotionsregulationsstudien wurden den Probanden aversive Bilder gezeigt und in den Placebostudien wurde den Probanden ein Schmerzreiz zugefügt. Die 
Inselrindenaktivität könnte somit durch das (restliche) Auf und Ab der vom stetigen negativen Reiz ausgelösten Erregung erzeugt worden sein (Diekhof et al. 2011a).

Die Inselrinde ist auch ein Teil des reaktionshemmenden Systems (Nakata et al. 2008). In den Emotionsregulationsstudien wurde die Gefühlsreaktion der Probanden und in den Placebostudien die Schmerzwahrnehmung gehemmt.

Ebenso ist eine Inselrindenaktivität auffällig bei der Erwartung unangenehmer Ereignisse oder bei der Vorbereitung auf unklare Situationen, die einen möglicherweise negativen Ausgang vermuten lassen (Herwig et al. 2007). Auch die Probanden der Emotionsregulationsstudien erwarteten die Präsentation unangenehmer Bilder, bekamen jedoch die Aufgabe, die Bilder neu zum Positiven zu interpretieren. Die erhöhte Inselrindenaktivität in der Paaranalyse von Placebo- und Emotionsregulationsstudien ist möglicherweise auf den Zeitpunkt der Erwartung zurückzuführen. Auch die Probanden der Placebostudien hatten eine Erwartung bezüglich des Schmerzreizes. Sie erwarteten eine Reduktion des Schmerzes durch den analgetischen Effekt des Placebos.

Zusätzlich trägt die anteriore Inselrinde durch reziproke Verbindungen mit der Amygdala zur Vermittlung von angstinduzierter Erregung und negativem Gefühlsstatus bei (Anders et al. 2004, Augustine 1996). Möglicherweise unterstützt die während der Placeboeinnahme und der Emotionsregulation erhöhte anteriore Inselaktivierung die VMPFC-Funktion, indem sie bei der inneren Verarbeitung von durch aversive Reize hervorgerufenen Gefühlen hilft.

\subsection{Die Amygdala - Zentrum der Deaktivierung während Regulation negativer Emotionen}

Die Ergebnisse dieser Metaanalyse bestätigen frühere Erkenntnisse über die wichtige Funktion der Amygdala für die Angstwahrnehmung und die Wahrnehmung negativer Emotionen. Die Ergebnisse dieser Metaanalyse zeigten bei der Reduktion negativer Emotionen eine Deaktivierung im Bereich der Amygdala. 18 von 29 Studien der vorliegenden Metaanalyse trugen zu diesem Ergebnis bei. 


\subsubsection{Die Amygdala: Kurzer Einblick in Einfluss und Funktion}

Die Amygdala ist eine Gehirnstruktur im medialen Teil des Temporallappens und ein Teil des limbischen Systems. Sie spielt eine Rolle bei der Entstehung von Angst, Wut, Entspannung, bei der Bewertung und Wiedererkennung von Situationen oder auch Gesichtsausdrücken (Adolphs et al. 1994, Rasia-Filho et al. 2000) sowie bei der Analyse möglicher Gefahren (Costafreda et al. 2008). Die Amygdala teilt sich in vier Kerngruppen, phylogenetisch ältere (Nucleus corticalis, Nucleus centralis) und jüngere (Nucleus basalis, Nucleus lateralis) (Prometheus 2006), hat Einfluss auf unterschiedliche funktionelle Systeme wie z. B. die sensorische Reizverarbeitung und ist für die emotionale Bewertung von Informationen zuständig.

\subsubsection{Inkludierte Studien der Metaanalyse ohne Amygdala-Deaktivität}

Es war zu beobachten, dass nicht bei allen Studien der Metaanalyse eine Amygdala-Deaktivierung festgestellt werden konnte. Von 29 Studien, die zusätzlich zu den Gehirnaktivierungen auch Gehirndeaktivierungen untersuchten, waren es 11 Studien, die nicht von Gehirndeaktivierungen im Bereich der Amygdala berichteten.

Ein möglicher Grund dafür könnte sein, dass nach einer erfolgreich abgeschlossenen willentlichen Gefühlsregulation eine paradoxe Aktivitätssteigerung der Amygdala, im Sinne eines Rebounds nach einer Unterdrückung von Gefühlen, eintritt (Walter et al. 2009). Als eine weitere Erklärung könnte dienen, dass die Probanden nach der Regulation der negativen Emotionen und Neubewertung des Erlebten oder Präsentierten nachsinnten und grübelten (Walter et al. 2009). In diesem Fall ist anzunehmen, dass die Hirnaktivität fälschlicherweise zu spät und nicht während der Regulation der Emotionen, sondern nach erfolgter Regulierung gemessen wurde.

Zusätzlich besteht die Möglichkeit, dass manche Reize stärkere emotionale Erregung bei den Probanden auslösten und manche Stimuli eine moralische Beurteilung bei den Probanden induzierten und somit die Verarbeitung und Neubewertung des wahrgenommenen Reizes eine stärkere Amygdala-Hemmung verursachte (Bishop et al. 2004). Dadurch ist auch schwer zu beurteilen, ob die Amydala-Aktivitätsänderung dem Regulationserfolg zu Grunde liegt oder den Misserfolg einer vorherigen fehlgeschlagenen Erregung widerspiegelt (Ochsner und Gross 2005). 


\subsection{Grenzen dieser Metaanalyse}

Beim Betrachten der Ergebnisse dieser Metaanalyse ist es wichtig, gewisse Einschränkungen zu berücksichtigen.

Es ist nicht außer Acht zu lassen, dass einige Studien dieser Metaanalyse besondere Untersuchungsschwerpunkte setzten, nur bestimmte Gehirnregionen von besonderem Interesse (Regions of interests $=$ ROIs) betrachteten oder nicht das komplette Gehirn mit in die magnetresonanztomografischen Untersuchungen einbezogen (meist „,small volume corrections““, z. B. Kim und Hamann 2007). ROIs ermöglichen es, Analysen auf bestimmte Bereiche eines Datensatzes zu begrenzen. Dieses führt folglich dazu, dass die ROIs überbewertet werden, da andere Gehirnareale gar nicht betrachtet werden, die möglicherweise ebenfalls während der Gefühlsregulation Aktivierungen aufweisen. Insgesamt führten sieben der neun Studien über die Furchtextinktion ihre Analysen mit ROIs durch (Kalisch et al. 2006b, Lang et al. 2009, Milad et al. 2007, Schiller et al. 2008, Soliman et al. 2010, Spoormaker et al. 2010, Yagues et al. 2005). Bei den Studien über den Placeboeffekt waren es sieben der 13 Studien, die ROIs definierten (Bingel et al. 2006, Eippert et al. 2009, Kong et al. 2009, Kong et al. 2006, Sarinopoulos et al. 2006, Wager et al. 2004 (1),(2)) und bei den Emotionsregulationsstudien zählten 17 der 23 Studien zu den Studien, die ROIs bestimmten (Banks et al. 2007, Delgado et al. 2008, Domes et al. 2010, Drabant et al. 2008, Eippert et al. 2007, Harenski und Hamann 2006, Kalisch et al. 2006c, Kim und Hamann 2007, Koenigsberg et al. 2010, Mak et al. 2009, McRae et al. 2008, 2009, Modinos et al. 2010, Ochsner et al. 2002, 2004, Urry et al. 2006, Winecoff et al. 2010). Dabei ist auch zu erwähnen, dass von den 18 Studien, die eine Amygdala-Deaktivität bei der Reduktion von negativen Emotionen festgestellt haben, nur sechs ohne ROIs arbeiteten (Knight et al. 2004, Petrovic et al. 2005, Goldin et al. 2007, Herwig et al. 2007, Johnstone et al. 2007, Walter et al. 2009). Ebenso zeigte sich bei den 11 Studien, die während der Regulation negativer Emotionen eine vermehrte VMPFC-Aktivität feststellten, dass lediglich fünf Studien ihre Untersuchungen durchführten, ohne vorher ROIs festzulegen (Finger et al. 2008, Diekhof et al. 2011b, Petrovic et al. 2005, Watson et al. 2009, Johnstone et al. 2007). Außerdem sind unter den sieben Studien, die gleichzeitig von VMPFC-Aktivierungen und Deaktivierung der Amygdala während der Regulation negativer 
Emotionen berichteten, nur zwei Studien, die zuvor keine ROIs bestimmt haben (Petrovic et al. 2005, Johnstone et al. 2007).

Es muss noch einmal betont werden, dass mit dieser Metaanalyse nur die Regulation von negativen Reizen untersucht wurde. Somit sind die Ergebnisse nicht generell auf andere Formen emotionaler Erfahrungen anwendbar (Banks et al. 2004) und nicht auf die generelle Gefühlsregulation transferierbar. Zusätzlich wurde beobachtet, dass besonders der orbitofrontale Kortex beim Menschen individuelle Unterschiede aufweisen kann. Dies kann zu Problemen führen, wenn Koordinaten vieler Probanden auf ein Standardgehirn übertragen werden und generelle Aussagen zu Funktion und Struktur getroffen werden (Kringelbach und Rolls 2004). Weiterhin können Unterschiede in der Studiendurchführung (z.B. unterschiedliche fMRTs) oder Fehler der Studien, die in die Metaanalyse inkludiert wurden, wiederum zu möglichen Fehlern der Ergebnisse dieser Metanalyse geführt haben. Zusätzlich werden Studien, die signifikante Ergebnisse aufweisen häufiger veröffentlicht (,publication bias“), was zu einer Verfälschung von Ergebnissen einer Metaanalyse führen kann (Walker et al. 2008).

Die Ergebnisse der Furchtextinktionsstudien sind wegen der geringen Studienanzahl und der damit verbundenen geringen Anzahl an untersuchten Probanden nur beschränkt generalisierbar. Außerdem wurden in den Emotionsregulationsstudien Regulationsstrategien angewandt, die sich etwas voneinander unterschieden, und über die erfolgte Regulation der Emotionen wurde nur subjektiv berichtet. Es gab keine objektive Messmethode, die einen möglichen Regulationserfolg hätte widerspiegeln können (Banks et al. 2007). Lediglich die verminderte Aktivierung im Bereich des limbischen Systems konnte als Regulationserfolg angesehen werden (Harenski und Harmann 2005). Außerdem wurden nicht die unterschiedlichen emotionalen Reaktionsfähigkeiten der Probanden bedacht. Die Probanden konnten sich beispielsweise in der Fähigkeit, Emotionen zu regulieren, unterscheiden (Stein et al. 2007, Etkin et al. 2004, Canli 2004, Haas et al. 2007).

Ebenso muss noch einmal daran erinnert werden, dass nicht alle Studien während der Regulation negativer Emotionen eine Aktivierung im VMPFC und eine Deaktivierung der Amygdala gezeigt haben. In $64 \%$ der Fälle war eine VMPFC-Aktivierung mit gleichzeitiger AmygdalaDeaktivierung feststellbar.

Es darf ebenfalls nicht unerwähnt bleiben, dass eine unterschiedliche Anzahl an Studien je Studienbereich (Furchtextinktion, Placeboeffekt, Emotionsregulation) mit in die Metaanalyse 
integriert wurde und folglich eine unterschiedliche Gewichtung der Bereiche im Ergebnis stattgefunden hat. Jedoch zeigten mehrere Studien aus den verschiedenen Studienbereichen die gleiche Tendenz. Die Hypothese, dass in allen drei Versuchstypen emotionale Regulationsstrategien genutzt wurden, konnte bestätigt werden konnte, da unter den sieben Studien, die gleichzeitig von VMPFC-Aktivierungen und Deaktivierung der Amygdala während der Reduktion negativer Emotionen berichteten, eine Studie aus dem Bereich Furchtextinktion, drei aus dem Studienbereich der Placebobehandlung und drei aus dem Bereich der Emotionsregulation zu finden waren. Basierend auf der Anzahl an Studien pro Studienbereich ergibt sich ein Verhältnis von 1:3:3 (Furchtextinktion: Placeboeffekt: Emotionsregulation), im Vergleich zu dem Verhältnis, aus dem die Summe der 29 Studien, die von Deaktivierungen berichteten, zusammengesetzt ist, 4:10:15 (Furchtextinktion: Placeboeffekt: Emotionsregulation). Dieser Vergleich zeigt, dass in jedem Studienbereich und unter Berücksichtigung der Anzahl der Studien ein gleich stark bedeutendes Ergebnis festgestellt wurde, mit der Besonderheit, dass die Studien über den Placeboeffekt das Ergebnis mehr als die beiden anderen Studientypen beeinflusst haben.

Nur wenige Studien haben die funktionellen Verbindungen zwischen den beteiligten Gehirnregionen untersucht. Folglich wäre es interessant, die genauen Mechanismen in Zukunft näher zu erforschen. Dies würde weitere Aufklärung über den Gefühlskreislauf geben und die Regulationsfunktion des VMPFC bei der emotionalen Verarbeitung bestätigen. Inwieweit die weiteren Gehirnregionen, die studientypspezifisch zusätzlich verstärkte Aktivität zeigten, Funktionen während der unterschiedlichen Versuchssituationen wie der Furchtextinktion, der Placebobehandlung und der Emotionsregulation übernehmen, konnte durch diese Arbeit nicht herausgearbeitet werden.

\subsection{Ausblick und Bedeutung für die Klinik}

Es ist wichtig, die präfrontal-limbischen Verbindungen zu verstehen, um gesunde emotionale Vorgänge nachvollziehen zu können. Besonders wichtig sind diese Erkenntnisse für die Ermittlung der Ätiologie und Diagnosen von psychischen Erkrankungen (Radulescu und Mujica-Parodi 2009). Koenigs et al. (2008) zeigten, dass viele Kriegsveteranen aus dem Vietnamkrieg an einer Posttraumatischen Belastungsstörung (PTSD) litten und diese mit Störungen im Bereich des 
VMPFC oder der Amygdala verbunden war. Es wurde meist eine Gehirndeaktivierung im Bereich des VMPFC und eine verstärkte Aktivität der Amygdala beobachtet. Etkin und Wager (2007, S. 1484) teilten Koenigs' et al. (2008) Vermutungen und meinten, dass die verminderte Aktivität im VMPFC bei Patienten mit PTSD die Defizite im emotionalen Regulationsprozess widerspiegeln.

Bei psychischen Erkrankungen wie sozialen Angststörungen (Phan et al. 2006), spezifischen Phobien wie der Spinnenphobie (Dilger et al. 2003), Panikstörungen und Zwangsstörungen (van den Heuvel et al. 2005) wurden Aktivitätsveränderungen bzw. Aktivierungen im Bereich der Amygdala gefunden. Ebenso wurde bei Patienten mit Depressionen (Drevets et al. 1992) eine verstärkte Amygdala-Aktivität beobachtet, die auf Grund von Dysfunktionen der inhibitorischen Kontrollmechanismen im präfrontalen Kortex auftrat (Yamasue et al. 2004).

Auch die mäßige Fehlregulation von Gefühlserregungen ist mit Substanzmissbrauch oder mit Erkrankungen wie Persönlichkeitsstörungen assoziiert. Grundlage von bipolaren Störungen oder der Schizophrenie sind ebenso Störungen der Regulation von Emotionen (Radulescu und MujicaParodi 2009).

\subsubsection{Klinische Relevanz der Placebostudien dieser Metaanalyse}

Die Placebostudien dieser Metaanalyse sind für die Klinik von starker Bedeutung. Placebos sind allgegenwärtig in der modernen Medizin, auch in klinischen Feldern wie Parkinsonerkrankungen, Depressionen und Schmerz (Benedetti et al. 2005, Enck et al. 2008, Price et al. 2008). Fregni et al. (2006) behandelten Patienten, die an Parkinson erkrankt waren mit dem Medikament Levodopa, alternativ mit einer Placebopille sowie mit vorgetäuschter transkranieller magnetischer Stimulation. Die Ergebnisse dieser Studie zeigten zwar nur eine objektive Besserung der Symptome der Patienten mit Levodopa-Behandlung, jedoch berichteten die Patienten mit der Placebobehandlung subjektiv von einer gleichen Besserung und einem Erfolg der Behandlung wie die Patienten der Levodopa-Gruppe (Kong et al. 2009).

Auch Placeboakupunkturmethoden bei Migränepatienten, Patienten mit Spannungskopfschmerz, chronischen Rückenschmerzen sowie mit Osteoarthritis führten zu ähnlichen Ergebnissen (Linde et al. 2007). 


\subsubsection{Klinische Relevanz der Emotionsregulationsstudien dieser Metaanalyse}

Für das menschliche Verhalten ist es wichtig, die Fähigkeit zu besitzen, Gefühle neu bewerten zu können, was die Emotionsregulationsstudien zu einem klinisch relevanten Thema macht.

Die „Realität“‘ durch verstärkte kontrollierte geistige Vorstellung neu zu interpretieren, ist Teil der kognitiven Verhaltenstherapie. Dies funktioniert wahrscheinlich über verstärkte regulierende präfrontale Mechanismen, die den sensorischen Kortex auf tieferer Ebene beeinflussen und die Erwartung einer reduzierten Angst induzieren und somit Auswirkung auf die Wahrnehmung haben (Hirsch und Holmes 2007).

Menschen, die häufig in ihrem täglichen Leben willentlich Neubewertungsstrategien nutzen, zeigen mehr zwischenmenschliche Fähigkeiten und sogar ein besseres psychisches und physischen Wohlbefinden (Gross und John 2003) und berichten von weniger negativen Gefühlsregungen und von weniger Depressionen (Groß und John 2003).

Im Vergleich zur Ablenkung, die nur einen kurzfristigen Erfolg aufweist, scheint die willentliche Neubewertung und Emotionsregulation einen erfolgreicheren und langfristigeren Effekt bei der Reduktion von negativen Gefühlen zu haben (Kalisch et al. 2006c, Chua et al. 1999, Kross und Ayduk 2008). Es ist $\mathrm{zu}$ vermuten, dass der VMPFC bei Personen, die häufiger Neubewertungsstrategien anwenden, stärker aktiv zu sein scheint, um einen hemmenden Effekt auf die Amygdala auszuüben.

\subsubsection{Klinische Relevanz der Furchtextinktionsstudien dieser Metaanalyse}

Mit Hilfe weiterer Forschung über das Erlernen der Furchtextinktion und damit verbundene neuronale Mechanismen werden Erklärungen von Krankheiten und Störungen wie Sucht, frontotemporale Demenz und andere psychische Störungen möglich sein, die eine Beeinträchtigung bei der Extinktion zeigen (Finger et al. 2008). Verspätete Extinktion oder Fehler bei der Löschung von erworbener Furcht könnten auch entscheidend für Krankheiten wie Angststörungen sein (Milad et al. 2006). Das Erlernen der Furchtextinktion ist daher wichtig für die Behandlung von Angststörungen. Auch Tierstudien über die Furchtextinktion versuchten neue therapeutische und pharmakologische Behandlungsmöglichkeiten $\mathrm{zu}$ untersuchen (Davis et al. 2006, LeDoux und Gorman 2001, Rauch et al. 2006). Ein vielversprechender Ansatz zur 
Behandlung von Angststörungen ist die kognitive Verhaltenstherapie, die die Konfrontation mit einem angsterzeugenden Hinweis in einem sicheren Umfeld beinhaltet und auf der Fähigkeit zur Extinktion basiert (Foa et al. 1999). Eine eingeschränkte Fähigkeit zur Extinktion kann jedoch zu Komplikationen bei der Angsttherapie führen, wenn das Angstgedächtnis in einer neuen Situation wieder aktiviert wird, obwohl die Löschung im therapeutischen Rahmen erfolgreich war (Lang et al. 2009). Dies bestätigen auch Orr SP et al. (2000) und Wessa und Flor (2007), die bei Patienten mit Angststörungen und Posttraumatischen Belastungsstörungen Defizite bzw. verminderte Aktivität im präfrontalen Bereich und im Bereich des Hippocampus, bei gleichzeitig fehlender Fähigkeit zur Extinktion, festgestellt haben. Der mPFC inhibiert in der Regel die Amygdala; fällt diese aktive Hemmung weg, reguliert die Amygdala frei die Gefühle. Dadurch entsteht über lange Zeit zusätzlich eine verstärkte Konditionierung des Angstgedächtnisses (Morgan et al. 1993).

\subsubsection{Geschlechterunterschiede in der Klinik}

Interessant sind auch die Geschlechterunterschiede bei der Häufigkeit von psychischen Erkrankungen. Frauen haben zweimal so häufig diagnostizierte affektive Störungen (Gater et al. 1998) und zeigen häufiger Depressionen als Männer (Kessler et al. 1993). Zusätzlich werden bei Frauen auch häufiger spezifische Phobien beobachtet (Fredrikson et al. 1996). Es wurde festgestellt, dass Frauen eine größere Neigung zum „Sinnieren“ besitzen, was zu negativen Emotionen führt (Thayer et al. 1994). Wegen möglicher Geschlechterunterschiede wurde in dieser Metaanalyse eine Untersuchung mit Probanden beiderlei Geschlechts durchgeführt. Diese zeigte ein ähnliches Ergebnis wie die Metaanalyse mit allen Emotionsregulationsstudien, auch solchen, in denen nur Frauen untersucht wurden. Folglich scheinen die Geschlechterunterschiede bei der Angstregulation und der Regulation negativer Emotionen eine untergeordnete Rolle zu spielen. Eine weitere Analyse mit nur einem Geschlecht würde möglicherweise kleinere Unterschiede bei der Furchtregulation zwischen den Geschlechtern aufdecken. 


\section{$5 \quad$ Zusammenfassung}

Angststörungen wie Posttraumatische Belastungsstörungen oder soziale Phobien sind Erkrankungen, die mit einer starken Beeinträchtigung des allgemeinen Gesundheitszustands eines Menschen einher gehen. Diese Arbeit stellte sich der Herausforderung, die Ergebnisse bereits publizierter Studien zu diesem Themenkomplex im Hinblick auf unterschiedliche Formen der Regulation negativer Emotionen und im Besonderen der Angstregulation zu vergleichen und mithilfe dieses Vergleichs ein mögliches übergreifendes Emotionsregulationszentrum zu identifizieren. Dazu wurden im Rahmen einer Pubmed-Recherche anhand definierter Einschlusskriterien Placebostudien, Furchtextinktionsstudien und Emotionsregulationsstudien selektiert und einer Metaanalyse unterzogen.

Es zeigte sich, dass der VMPFC als Regulationszentrum für negative Emotionen mit Verbindungen zum limbischen System, im Besonderen zur Amygdala, eine herausgehobene Funktion während der Emotionskontrolle einnimmt. Hierbei fiel auf, dass eine VMPFCAktivierung während der Angstregulation sowie der Reduktion einer negativen Empfindung von einer Amygdala-Deaktivierung begleitet war.

Zudem konnten beim Vergleich von Placebostudien und Emotionsregulationsstudien zwei weitere Regionen mit verstärkter Aktivität während des Regulationsprozesses von negativen Emotionen beobachtet werden - die rechte anteriore Inselrinde und der linke ACC.

Außerdem zeigten die Metaanalysen, dass zusätzlich zu dem VMPFC je nach Studientyp noch weitere Hirnregionen während der Regulation von Furcht und negativen Emotionen aktiviert waren, um zusätzlich Einfluss auf den Gefühlsprozess auszuüben. Es ist somit nicht auszuschließen, dass neben dem ventromedialen präfrontalen Gefühlsregulationsmechanismus, der auch in Tierstudien beobachtet wurde (Quirk und Beer 2006, Milad et al. 2006), auf höherer Ebene beim Menschen noch weitere Kontrollmechanismen existieren (Delgado et al. 2008), die wahrscheinlich die ventromedialen präfrontalen Mechanismen ergänzen (Diekhof et al. 2011a).

Es bleibt festzuhalten, dass der VMPFC und die Amygdala in einem engen funktionellen Zusammenhang stehen.

Zukünftige Forschungsprojekte sollten sich der genaueren Erforschung der Verbindung zwischen dem VMPFC und der Amygdala während der generellen Gefühlsregulation widmen und die 
Funktionen der anderen aktivierten studienbereichsspezifischen Gehirnregionen während der Regulation der negativen Emotionen in die Untersuchung miteinbeziehen.

Solche Forschungsergebnisse könnten dazu beitragen, bei Erkrankungen wie Angststörungen neue und erfolgversprechende Therapiemöglichkeiten zu finden. 
6. Literaturverzeichnis

\section{Literaturverzeichnis}

Adolphs R, Tranel D, Damasio H, Damasio A (1994): Impaired recognition of emotion in facial expressions following bilateral damage to the human amygdala. Nature $\underline{372}, 669-672$

Akirav I, Maroun M (2007): The role of the medial prefrontal cortex-amygdala circuit in stress effects on the extinction of fear. Neural Plast 2007, 30873, 1- 11

Amanzio M, Benedetti F (1999): Neuropharmacological dissection of placebo analgesia: expectation-activated opioid systems versus conditioning-activated specific subsystems. J Neurosci $\underline{19}, 484-494$

Amodio DM, Frith CD (2006): Meeting of minds: the medial frontal cortex and social cognition. Nat Rev Neurosci 7, 268- 277

Anders S, Lotze M, Erb M, Grodd W, Birbaumer N (2004): Brain activity underlying emotional valence and arousal: a response-related fMRI study. Hum Brain Mapp 23, 200-209

Aouizerate B, Rotgé JY, Bioulac B, Tignol J (2007): Present contribution of neurosciences to a new clinical reading of obsessive-compulsive disorder. Encephale 33, 203- 310

Atlas LY, Bolger N, Lindquist MA, Wager TD (2010): Brain mediators of predictive cue effects on perceived pain. J Neurosci $\underline{30}, 12964-12977$

Augustine JR (1996): Circuitry and functional aspects of the insular lobe in primates including humans. Brain Res Rev 22, 229- 244

Banks SJ, Eddy KT, Angstadt M, Nathan PJ, Phan KL (2007): Amygdala-frontal connectivity during emotion regulation. Scan $\underline{2}, 303-312$

Bar M (2007): The proactive brain: using analogies and associations to generate predictions. Trends Cogn Sci 11, 280- 289

Bar M (2009): The proactive brain: memory for predictions. Philos Trans R Soc Lond B Biol Sci $\underline{364}, 1235-1243$ 
Bar M, Aminoff E, Ishai A (2008): Famous faces activate contextual associations in the parahippocampal cortex. Cereb Cortex $\underline{18}$, 1233-1238

Bechara A, Damasio AR, Damasio H, Anderson SW (1994): Insensitivity to future consequences following damage to human prefrontal cortex. Cognition $\underline{50,7-15}$

Bechara A, Damasio H, Damasio AR, Lee GP (1999): Different contributions of the human amygdala and ventromedial prefrontal cortex to decision-making. J Neurosci $\underline{19}, 5473-5481$

Benedetti F, Arduino C, Amanzio M (1999): Somatotopic activation of opioid systems by targetdirected expectations of analgesia. J Neurosci $\underline{19}, 3639-3648$

Benedetti F, Pollo A, Lopiano L, Lanotte M, Vighetti S, Rainero I (2003): Conscious Expectation and Unconscious Conditioning in Analgesic, Motor, and Hormonal Placebo/Nocebo Responses. J Neurosci $\underline{23}, 4315-4323$

Benedetti F, Mayberg HS, Wager TD,Stohler CS, Zubieta J (2005): Neurobiological Mechanisms of the Placebo Effect. J Neurosci 25, 10390- 10402

Benuzzi F, Lui F, Duzzi D, Nichelli PF, Porro CA (2008): Does it look painful or disgusting? Ask your parietal and cingulate cortex. J Neurosci $\underline{28}$, 923- 931

Bermpohl F, Pascual-Leone A, Amedi A, Merabet LB, Fregni F, Gaab N, Alsop D, Schlaug G, Northoff G (2006): Dissociable networks for the expectancy and perception of emotional stimuli in the human brain. Neuroimage $\underline{30}, 588-600$

Berretta S, Pantazopoulos H, Caldera M, Pantazopoulos P, Pare D (2005): Infralimbic cortex activation increases c-Fos expression in intercalated neurons of the amygdala. Neuroscience $\underline{132}$, 943-953

Bingel U, Lorenz J, Schoell E, Weiller C, Büchel C (2006): Mechanisms of placebo analgesia: rACC recruitment of a subcorticalantinociceptive network. Pain $\underline{120}, 8-15$

Bishop S, Duncan J, Brett M, Lawrence AD (2004): Prefrontal cortical function and anxiety: controlling attention to threat-related stimuli. Nat Neurosci $\underline{7}, 184-188$ 
Boly M, Balteau E, Schnakers C, Degueldre C, Moonen G, Luxen A, Phillips C, Peigneux P, Maquet P, Laureys S (2007): Baseline brain activity fluctuations predict somatosensory perception in humans. Proc Natl Acad Sci U S A $\underline{104}$, 12187- 12192

Bos W, McClure SM, Harris LT, Fiske ST, Cohen JD (2007): Dissociating affective evaluation and social cognitive processes in the ventral medial prefrontal cortex. Cogn Affect Behav Neurosci 7, 337- 346

Botvinick MM (2007): Conflict monitoring and decision making: reconciling two perspectives on anterior cingulate function. Cogn Affect Behav Neurosci 7, 356- 366

Bouton ME (2004): Context and behavioral processes in extinction. Learn Mem $\underline{11}$, 485- 494

Bower GH (1981): Mood and memory. Am Psychol 36, 129- 148

Brown MR, Lebel RM, Dolcos F, Wilman AH, Silverstone PH, Pazderka H, Fujiwara E, Wild TC, Carroll AM, Hodlevskyy O (2012): Effects of emotional context on impulse control. Neuroimage $\underline{15 ; 63(1)}, 434-446$

Brown S, Ingham RJ, Ingham JC, Laird AR, Fox PT (2005): Stuttered and fluent speech production: an ALE meta-analysis of functional neuroimaging studies. Hum Brain Mapp 25, 105117

Büchel C, Bornhovd K, Quante M, Glauche V, Bromm B, Weiller C (2002): Dissociable neural responses related to pain intensity, stimulus intensity, and stimulus awareness within the anterior cingulate cortex: a parametric single-trial laser functional magnetic resonance imaging study. J Neurosci $\underline{22}, 970-976$

Cacioppo JT, Gernston GG, Larsen JT, Poehlmann KM, Ito TA: The psychophysiology of emotion. In: Handbook of Emotions. Lewis M, Haviland-Jones JM, vol 2, Guilford Press, New York 2000, $173-191$

Canli T (2004): Functional brain mapping of extraversion and neuroticism: learning from individual differences in emotion processing. J Pers $\underline{72}, 1105-1132$

Carlsson K, Petrovic P, Skare S, Petersson KM, Ingvar M (2000): Tickling expectations: neural processing in anticipation of a sensory stimulus. J Cogn Neurosci $\underline{12}, 691-703$ 
Caspers S, Zilles K, Laird AR, Eickhoff SB (2010): ALE meta-analysis of action observation and imitation in the human brain. Neuroimage $\underline{50}$, 1148- 1167

Chein JM, Fissell K, Jacobs S, Fiez JA (2002): Functional heterogeneity within Broca's area during verbal working memory. Physiol Behav $\underline{77}$, 635- 639

Chua P, Krams M, Toni I, Passingham R, Dolan R (1999): A functional anatomy of anticipatory anxiety. Neuroimage $\underline{9}, 563-571$

Ciaramelli E, Muccioli M, Làvadas E, di Pellegrino G (2007): Selective deficit in personal moral judgment following damage to ventromedial prefrontal cortex. Scan $\underline{2}, 84-92$

Clark L, Bechera A, Damasio H, Aitken MRF, Sahakian BJ, Robbins TW (2008): Differential effects of insular and ventromedial prefrontal cortex lesions on risky decision- making. Brain $\underline{131}$, $1311-1322$

Colloca L, Petrovic P, Wager TD, Ingvar M, Benedetti F (2010): How the number of learning trials affects placebo and nocebo responses. Pain $\underline{151}, 430-439$

Corbetta M, Shulman GL (2002): Control of goal- directed and stimulus- driven attention in the brain. Nat Rev Neurosci $\underline{3}, 201-215$

Corbetta M, Patel G, Shulman GL (2008): The reorienting system of the human brain: from environment to theory of mind. Neuron $\underline{58}, 306-324$

Corcoran KA, Quirk GJ (2007): Recalling safety: cooperative functions of the ventromedial prefrontal cortex and the hippocampus in extinction. CNS Spectr $\underline{12}$, 200- 206

Costafreda SG, Brammer MJ, David AS, Fu CH (2008): Predictors of amygdala activation during the processing of emotional stimuli: a metaanalysis of 385 PET and fMRI studies. Brain Res Rev $\underline{58}, 57-70$

Craig AD (2009): How do you feel—now? The anterior insula and human awareness. Nat Rev Neurosci $\underline{10}, 59-70$

Critchley HD, Wiens S, Rotshtein P, Öhman A, Dolan RJ (2004): Neural systems supporting interoceptive awareness. Nat Neurosci $\underline{7}, 189-195$ 
D'Argembeau A, Ruby P, Collette F, Degueldre C, Balteau E, Luxen A, Maquet P, Salmon E (2007): Distinct regions of the medial prefrontal cortex are associated with selfreferential processing and perspective taking. J Cogn Neurosci $\underline{19}$, 935- 944

Davidson RJ (2002): Anxiety and affective style: role of prefrontal cortex and amygdala. Biol Psychiatry $\underline{51}, 68-80$

Davidson RJ, Putnam KM, Larson CL (2000): Dysfunction in the neural circuitry of emotion regulation: a possible prelude to violence. Science $\underline{289}, 591-594$

Davidson RJ, Lewis DA, Alloy LB, Amaral DG, Bush G, Cohen JD, Drevets WC, Farah MJ, Kagan J, McClelland JL, Nolen-Hoeksema S, Peterson BS (2002): Neural and behavioral substrates of mood and mood regulation. Biol Psychiatry $\underline{52}, 478$ - 502

Davis M, Ressler K, Rothbaum BO, Richardson R (2006): Effects of D-cycloserine on extinction: translation from preclinical to clinical work. Biol Psychiatry $\underline{60}, 369-375$

de Araujo IE, Rolls ET, Velazco MI, Margot C, Cayeux I (2005): Cognitive modulation of olfactory processing. Neuron $\underline{46}, 671-679$

Decety J, Lamm C (2007): The role of the right temporoparietal junction in social interaction: how low-level computational processes contribute to meta-cognition. Neuroscientist $\underline{13}, 580-593$

Delamater AR (2004): Experimental extinction in Pavlovian conditioning: behavioural and neuroscience perspectives. Q J Exp Psychol B 57, 97- 132

Delgado MR, Nearing KI, Ledoux JE, Phelps EA (2008): Neural circuitry underlying the regulation of conditioned fear and its relation to extinction. Neuron $\underline{59}, 829-838$

DerSimonian R, Laird N (1986): Mata- Analysis in Clinical Trials. Control Clin Trials $\underline{7}$, 177- 188

Diekhof EK, Geier K, Falkai P, Gruber O (2011a): Fear is only as deep as the mind allows: a coordinate-based meta-analysis of neuroimaging studies on the regulation of negative affect. Neuroimage $\underline{58}, 275-285$

Diekhof EK, Kipshagen HE, Falkai P, Dechent P, Baudewig J, Gruber O (2011b): The power of 
imagination - How anticipatory mental imagery alters perceptual processing of fearful facial expressions. Neuroimage $\underline{54}, 1703-1714$

Dilger S, Straube T, Mentzel HJ, Fitzek C, Reichenbach JR, Hecht H, Krieschel S, Gutberlet I, Miltner WH (2003): Brain activation to phobia-related pictures in spider phobic humans: an eventrelated functional magnetic resonance imaging study. Neurosci Lett $\underline{348}, 29-32$

Domes G, Schulze L, Böttger M, Grossmann A, Hauenstein K, Wirtz PH, Heinrichs M, Herpertz SC (2010): The Neural Correlates of Sex Differences in Emotional Reactivity and Emotion Regulation. Hum Brain Mapp 31, 758- 769

Drabant EM, McRae K, Manuck SB, Hariri AR, Gross JJ (2008): Individual Differences in Typical Reappraisal Use Predict Amygdala and Prefrontal Responses. Biol Psychiatry 65, 367- 373

Drevets WC (2000): Neuroimaging studies of mood disorders. Biol Psychiatry $\underline{48}$, 813- 829

Drevets WC, Videen TO, Price JL, Preskorn SH, Carmichael ST, Raichle ME (1992): A functional anatomical study of unipolar depression. J Neurosci $\underline{12}, 3628-3641$

Eickhoff SB, Laird AR, Grefkes C, Wang LE, Zilles K, Fox PT (2009): Coordinate-based activation likelihood estimation meta-analysis of neuroimaging data: a random-effects approach based on empirical estimates of spatial uncertainty. Hum Brain Mapp 30, 2907- 2926

Eippert F, Veit R, Weiskopf N, Erb M, Birbaumer N, Anders S (2007): Regulation of Emotional Responses Elicited by Threat-Related Stimuli. Hum Brain Mapp 28, 409- 423

Eippert F, Bingel U, Schoell ED, Yacubian J, Klinger R, Lorenz J, Büchel C (2009): Activation of the opioidergic descending pain control system underlies placebo analgesia. Neuron $\underline{63}, 533-543$

Enck P, Benedetti F, Schedlowski M (2008): New insights into the placebo and nocebo responses. Neuron $\underline{59}, 195-206$

Etkin A, Wager TD (2007): Functional neuroimaging of anxiety: a meta-analysis of emotional processing in PTSD, social anxiety disorder, and specific phobia. Am J Psychiatry 164, 1476- 1488 
Etkin A, Klemenhagen KC, Dudman JT, Rogan MT, Hen R, Kandel ER, Hirsch J (2004): Individual differences in trait anxiety predict the response of the basolateral amygdala to unconsciously processed fearful faces. Neuron $\underline{44}, 1043-1055$

Etkin A, Egner T, Peraza DM, Kandel ER, Hirsch J (2006): Resolving emotional conflict: a role for the rostral anterior cingulate cortex in modulating activity in the amygdala. Neuron $\underline{51}, 871-$ 882

Etkin A, Egner T, Kalisch R (2011): Emotional processing in anterior cingulate and medial prefrontal cortex. Trends Cogn Sci $\underline{15}, 85-93$

Fanselow MS (1994): Neural organization of the defensive behavior system responsible for fear. Psychon Bull Rev 1, 429- 438

Finger EC, Mitchell DG, Jones M, Blair RJ (2008): Dissociable roles of medial orbitofrontal cortex in human operant extinction learning. Neuroimage $\underline{43}, 748-755$

Fitzgerald PB, Laird AR, Maller J, Daskalakis ZJ (2008): A meta-analytic study of changes in brain activation in depression. Hum Brain Mapp 29, 683- 695

Fredrikson M, Annas P, Fischer H, Wik G (1996): Gender and age differences in the prevalence of specific fears and phobias. Behav Res Ther $\underline{34}, 33-39$

Fregni F, Boggio PS, Bermpohl F, Maia F, Rigonatti SP, Barbosa ER, Pascual-Leone A (2006): Immediate placebo effect in Parkinson's disease--is the subjective relief accompanied by objective improvement? Eur Neurol $\underline{56}, 222-229$

Freund W, Stuber G, Wunderlich AP, Schmitz B (2007): Cortical correlates of perception and suppression of electrically induced pain. Somatosens Mot Res 24, 203- 212

Fuster JM (2002): Frontal lobe and cognitive development. J Neurocytol 31, 373- 385

Gallagher HL, Happe F, Brunswick N, Fletcher PC, Frith U, Frith CD (2000): Reading the mind in cartoons and stories: An fMRI study of 'theory of mind' in verbal and nonverbal tasks. Neuropsychologia $\underline{38}, 11-21$ 
Gater R, Tansella M, Korten A, Tiemens BG, Mavreas VG, Olatawura MO (1998): Sex differences in the prevalence and detection of depressive and anxiety disorders in general health care settings: report from the World Health Organization Collaborative Study on Psychological Problems in General Health Care. Arch Gen Psychiatry 55, 405- 413

Ghuman AS, Bar M, Dobbins IG, Schnyer DM (2008): The effects of priming on frontal-temporal communication. Proc Natl Acad Sci U S A $\underline{105}$, 8405- 8409

Glahn DC, Ragland JD, Abramoff A, Barrett J, Laird AR, Bearden CE, Velligan DI (2005): Beyond hypofrontality: a quantitative meta-analysis of functional neuroimaging studies of working memory in schizophrenia. Hum Brain Mapp 25, 60- 69

Goffaux, P, Redmond WJ, Rainville P, Marchand S (2007): Descending analgesia-when the spine echoes what the brain expects. Pain $\underline{130}, 137-143$

Goldin PR, McRae K, Ramel W, Gross JJ (2007): The Neural Bases of Emotion Regulation: Reappraisal and Suppression of Negative Emotion. Biol Psychiatry 63, 577- 586

Gottfried JA, Dolan RJ (2004): Human orbitofrontal cortex mediates extinction learning while accessing conditioned representations of value. Nat Neurosci $\underline{7}, 1144-1152$

Gottfried JA, O'Doherty J, Dolan RJ (2003): Encoding predictive reward value in human amygdala and orbitofrontal cortex. Science $\underline{301}, 1104-1107$

Grabenhorst F, Rolls ET (2011): Value, pleasure and choice in the ventral prefrontal cortex. Trends Cogn Sci $\underline{15}, 56-67$

Grabenhorst F, Rolls ET, Bilderbeck A (2008): How cognition modulates affective responses to taste and flavor: top-down influences on the orbitofrontal and pregenual cingulate cortices. Cereb Cortex $\underline{18}, 1549-1559$

Gracely RH, Dubner R, Wolskee PJ, Deeter WR (1983): Placebo and naloxone can alter postsurgical pain by separate mechanisms. Nature $\underline{306}, 264-265$

Grevert P, Albert LH, Goldstein A (1983): Partial antagonism of placebo analgesia by naloxone. 
6. Literaturverzeichnis

Pain $\underline{16}, 129-143$

Grosbras MH, Laird AR, Paus T (2005): Cortical regions involved in eye movements, shifts of attention, and gaze perception. Hum Brain Mapp 25, 140- 154

Gross JJ (1998): The emerging field of emotion regulation: An integrative review. Rev Gen Psychol 2, 271-299

Gross JJ (2002): Emotion regulation: Affective, cognitive, and social consequences. Psychophysiology $\underline{39}, 281-291$

Gross JJ, John OP (2003): Individual differences in two emotion regulation processes: Implications for affect, relationships, and well-being. J Pers Soc Psychol $\underline{85}, 348$ - 362

Gruber O, Melcher T, Diekhof EK, Karch S, Falkai P, Goschke T (2009): Brain mechanisms associated with background monitoring of the environment for potentially significant sensory events. Brain Cogn $\underline{69}, 559-564$

Güroğlu B, Haselager GJ, van Lieshout CF, Takashima A, Rijpkema M, Fernández G (2008): Why are friends special? Implementing a social interaction simulation task to probe the neural correlates of friendship. NeuroImage $\underline{39}$, 903- 910

Gusnard DA, Akbudak E, Shulman GL, Raichle ME (2001): Medial prefrontal cortex and selfreferential mental activity: Relation to a default mode of brain function. Proc Natl Acad Sci USA $\underline{98}, 4259-4264$

Haas BW, Omura K, Constable RT, Canli T (2007): Emotional conflict and neuroticism: personality- dependent activation in the amygdala and subgenual anterior cingulate. Behav Neurosci 121, 249- 256

Hakamata Y, Matsuoka Y, Inagaki M, Nagamine M, Hara E, Imoto S, Murakami K, Kim Y, Uchitomi Y (2007): Structure of orbitofrontal cortex and its longitudinal course in cancerrelated posttraumatic stress disorder. Neurosci Res $\underline{59}$, 383- 389 
Happe F, Ehlers S, Fletcher P, Frith U, Johansson M, Gillberg C, Dolan R, Frackowiak R, Frith C (1996): 'Theory of mind' in the brain. Evidence from a PET scan study of Asperger syndrome. NeuroReport $\underline{8}, 197-201$

Harenski CL, Hamann S (2006): Neural correlates of regulating negative emotions related to moral violations. NeuroImage $\underline{30}, 313-324$

Hauber W, Sommer S (2009): Prefrontostriatal circuitry regulates effort-related decision making. Cereb Cortex 19, 2240- 2247

Herwig U, Kaffenberger T, Baumgartner T, Jäncke L (2007): Neural correlates of a 'pessimistic' attitude when anticipating events of unknown emotional valence. Neuroimage $\underline{34}, 848-858$

Hirsch C, Holmes EA (2007): Mental imagery in anxiety disorders. Psychiatry $\underline{6}, 161-165$

Holland PC (1990): Event representation in Pavlovian conditioning: image and action. Cognition $\underline{37}, 105-131$

Holmes EA, Brewin CR, Hennessy RG (2004): Trauma Films, Information Processing, and Intrusive Memory Development. J Exp Psychol 133, 3- 22

Izquierdo A, Murray EA (2005): Opposing effects of amygdala and orbital prefrontal cortex lesions on the extinction of instrumental responding in macaque monkeys. Eur J Neurosci 22, 2341-2346

Jackson DC, Malmstadt JR, Larson CL, Davidson RJ (2000): Suppression and enhancement of emotional responses to unpleasant pictures. Psychophysiology $\underline{37}$, 515- 522

JohnstoneT, van Reekum CM, Urry HL, Kalin NH, Davidson RJ (2007): Failure to regulate: counterproductive recruitment of top-down prefrontal-subcortical circuitry in major depression. $\mathbf{J}$ Neurosci $\underline{27}, 8877-8884$

Kalisch R, Wiech K, Critchley HD, Seymour B, O’Doherty JP, Oakley DA, Allen P, Dolan R J (2005): Anxiety reduction through detachment: Subjective, physiological and neural effects. Journal of Cognitive Neuroscience $\underline{17}, 874-883$ 
Kalisch R, Wiech K, Critchley HD, Dolan RJ (2006a): Levels of appraisal: a medial prefrontal role in high-level appraisal of emotional material. Neuroimage $\underline{30}$, 1458- 1466

Kalisch R, Korenfeld E, Stephan KE, Weiskopf N, Seymour B, Dolan RJ (2006b): Contextdependent human extinction memory is mediated by a ventromedial prefrontal and hippocampal network. J Neurosci 26, 9503-9511

Kalisch R, Wiech K, Herrmann K, Dolan RJ (2006c): Neural Correlates of Self-distraction from Anxiety and a Process Model of Cognitive Emotion Regulation. J Cogn Neurosci 18, 1266- 1276

Kellermann TS, Sternkopf MA, Schneider F, Habel U, Turetsky BI, Zilles K, Eickhoff SB (2011): Modulating the processing of emotional stimuli by cognitive demand. Soc Cogn Affect Neurosci 7, $263-273$

Keltner D, Kring A (1998): Emotion, social function, and psychopathology. Rev Gen Psychol $\underline{2}$, $320-342$

Keltner JR, Furst A, Fan C, Redfern R, Inglis B, Fields HL (2006): Isolating the modulatory effect of expectation on pain transmission: a functional magnetic resonance imaging study. $\mathrm{J}$ Neurosci $\underline{26}, 4437-4443$

Kessler RC, McGonagle KA, Swartz M, Blazer DG, Nelson CB (1993): Sex and depression in the National Comorbidity Survey. I: Lifetime prevalence, chronicity and recurrence. J Affect Disord $\underline{29}, 85-96$

Kim SH, Hamann S (2007): Neural correlates of positive and negative emotion regulation. J Cogn Neurosci $\underline{19}, 776-798$

Knight DC, Smith CN, Cheng DT, Stein EA, Helmstetter FJ (2004): Amygdala and hippocampal activity during acquisition and extinction of human fear conditioning. Cogn Affect Behav Neurosci 4, 317- 325

Koenigs M, Huey ED, Raymont V, Cheon B, Solomon J, Wassermann EM, Grafman J (2008): Focal brain damage protects against post-traumatic stress disorder in combat veterans. Nat Neurosci $\underline{11}, 232$ - 237 
Koenigsberg HW, Fan J, Ochsner KN, Liu X, Guise K, Pizzarello S, Dorantes C, Tecuta L, Guerreri S, Goodman M, New A, Flory J, Siever LJ (2010): Neural correlates of using distancing to regulate emotional responses to social situations.Neuropsychologia $\underline{48}, 1813-1822$

Koler JI: Der Einfluss von $\Delta 9$-THC auf Lernen und Gedächtnis. Psychologische Diplomarbeit. Heidelberg 2006

Kong J, Gollub RL, Rosman IS, Webb JM, Vangel MG, Kirsch I, Kaptchuk TJ (2006): Brain activity associated with expectancyenhanced placebo analgesia as measured by functional magnetic resonance imaging. J Neurosci 26, 381- 388

Kong J, Kaptchuk TJ, Polich G, Kirsch I, Vangel M, Zyloney C, Rosen B, Gollub R (2009): Expectancy and treatment interactions: a dissociation between acupuncture analgesia and expectancy evoked placebo analgesia. Neuroimage $\underline{45}$, 940- 949

Kringelbach ML (2005): The human orbitofrontal cortex: linking reward to hedonic experience. Nat Rev Neurosci $\underline{6}, 691-702$

Kringelbach ML, Rolls ET (2003): Neural correlates of rapid reversal learning in a simple model of human social interaction. Neuroimage 20, 1371- 1383

Kringelbach ML, Rolls ET (2004): The functional neuroanatomy of the human orbitofrontal cortex: evidence from neuroimaging and neuropsychology. Prog Neurobiol 르, 341- 372

Kross E, Ayduk O (2008): Facilitating adaptive emotional analysis: distinguishing distancedanalysis of depressive experiences from immersed-analysis and distraction. Pers Soc Psychol Bull $\underline{34}, 924-938$

Kross E, Davidson M, Weber J, Ochsner K (2009): Coping with emotions past: the neural bases of regulating affect associated with negative autobiographical memories. Biol Psychiatry $\underline{65}, 361-366$ Laird AR (2009): User Manual for Ginger ALE 2.1. http://brainmap.org

Laird AR, McMillan KM, Lancaster JL, Kochunov P, Turkeltaub PE, Pardo JV, Fox PT (2005a): A comparison of label-based review and activation likelihood estimation in the Stroop task. Hum Brain Mapp 25, 6- 21 
Laird AR, Fox PM, Price CJ, Glahn DC, Uecker AM, Lancaster JL, Turkeltaub PE, Kochunov P, Fox PT (2005b): ALE Meta-Analysis: Controlling the False Discovery Rate and Performing Statistical Contrasts. Hum Brain Mapp 25, 155- 164

Laird AR, Eickhoff SB, Kurth F, Fox PM, Uecker AM, Turner JA, Robinson JL, Lancaster JL, Fox PT (2009): ALE Meta-Analysis Workflows Via the Brainmap Database: Progress Towards A Probabilistic Functional Brain Atlas. Front Neuroinform 3: 23, 1- 11

Lamm C, Decety J, Singer T (2011): Meta-analytic evidence for common and distinct neural networks associated with directly experienced pain and empathy for pain. Neuroimage $\underline{54}, 2492-$ 2502

Lancaster JL, Tordesillas-Gutiérrez D, Martinez M, Salinas F, Evans A, Zilles K, Mazziotta JC, Fox PT (2007): Bias between MNI and Talairach coordinates analyzed using the ICBM-152 brain template. Hum Brain Mapp 28, 1194- 1205

Lang S, Kroll A, Lipinski SJ, Wessa M, Ridder S, Christmann C, Schad LR, Flor H (2009): Context conditioning and extinction in humans: differential contribution of the hippocampus, amygdala and prefrontal cortex. Eur J Neurosci 29, 823- 832

LeDoux JE, Gorman JM (2001): A call to action: overcoming anxiety through active coping. Am J Psychiatry $\underline{158}, 1953-1955$

Levenson RW: Autonomic specificity and emotion. In: Handbook of Affective Sciences; Davidson RJ, Scherer KR, Goldsmith HH; Oxford University Press. New York 2003, 212- 224

Liberzon I, Zubieta JK, Fig LM, Phan KL, Koeppe RA, Taylor SF (2002): mu-Opioid receptors and limbic responses to aversive emotional stimuli. Proc Natl Acad Sci USA $\underline{99}, 7084-7089$

Linde K, Witt CM, Streng A, Weidenhammer W, Wagenpfeil S, Brinkhaus B, Willich SN, Melchart D (2007): The impact of patient expectations on outcomes in four randomized controlled trials of acupuncture in patients with chronic pain. Pain $\underline{128}, 264-271$

MacDonald AW 3rd, Cohen JD, Stenger VA, Carter CS (2000): Dissociating the role of the dorsolateral prefrontal and anterior cingulate cortex in cognitive control. Science $\underline{288}, 1835-1838$ Mak AK, Hu ZG, Zhang JX, Xiao ZW, Lee TM (2009): Neural correlates of regulation of positive 
6. Literaturverzeichnis

and negative emotions: an fmri study. Neurosci Lett $\underline{457,101-106}$

Maren S, Quirk GJ (2004): Neuronal signalling of fear memory. Nat Rev Neurosci $\underline{5}$, 844-852

Mather M, Canli T, English T, Whitfield S, Wais P, Ochsner K, Gabrieli JD, Carstensen LL (2004): Amygdala responses to emotionally valenced stimuli in older and younger adults. Psychol Sci $\underline{15}$, 259- 263

Mathews A (2004): On the malleability of emotional encoding. Behav Res Ther $\underline{42}$, 1019- 1036

Matre D, Casey KL, Knardahl S (2006): Placebo-induced changes in spinal cord pain processing. J Neurosci 26, 559- 563

McClure SM, Li J, Tomlin D, Cypert KS, Montague LM, Montague PR (2004): Neural correlates of behavioral preference for culturally familiar drinks. Neuron $\underline{44}$, 379- 387

McRae K, Ochdner KN, Mauss IB, Gabrieli JJD, Gross JJ (2008): Gender differences in emotion regulation: an fMRI study of cognitive reappraisal. Group Process Inerg 11, 143- 162

McRae K, Hughes B, Chopra S, Gabrieli JD, Gross JJ, Ochsner KN (2009): The neural bases of distraction and reappraisal. J Cogn Neurosci $\underline{22}, 248-262$

Mechias ML, Etkin A, Kalisch R (2010): A meta-analysis of instructed fear studies: implications for conscious appraisal of threat. Neuroimage $\underline{49}, 1760-1768$

Mellers, BA (2000): Choice and the relative pleasure of consequences. Psychol Bull 126, 910-924

Menzies L, Chamberlain SR, Laird AR, Thelen SM, Sahakian BJ, Bullmore ET (2008): Integrating evidence from neuroimaging and neuropsychological studies of obsessive-compulsive disorder: the orbitofronto-striatal model revisited. Neurosci Biobehav Rev $\underline{32}, 525-549$

Milad MR, Quirk GJ (2002): Neurons in medial prefrontal cortex signal memory for fear extinction. Nature $\underline{420}, 70-74$

Milad MR, Rauch SL, Pitman RK, Quirk GJ (2006): Fear extinction in rats: implications for human 
6. Literaturverzeichnis

brain imaging and anxiety disorders. Biol Psychol $\underline{73}, 61-71$

Milad MR, Wright CI, Orr SP, Pitman RK, Quirk GJ, Rauch SL (2007): Recall of fear extinction in humans activates the ventromedial prefrontal cortex and hippocampus in concert. Biol Psychiatry $\underline{62}, 446-454$

Mineka S, Watson D, Clark LA (1998): Comorbidity of anxiety and unipolar mood disorders. Annu Rev Psychol 49, 377- 412

Miller EK, Cohen JD (2001): An integrative theory of prefrontal cortex function. Annu Rev Neurosci 24, 167- 202

Minzenberg MJ, Laird AR, Thelen S, Carter CS, Glahn DC (2009): Meta-analysis of 41 functional neuroimaging studies of executive function in schizophrenia. Arch Gen Psychiatry $\underline{66}, 811-822$

Mitchell JP, Macrae CN, Banaji MR (2006): Dissociable medial prefrontal contributions to judgments of similar and dissimilar others. Neuron $\underline{50}, 655-663$

Mobbs D, Weiskopf N, Lau HC, Featherstone E, Dolan RJ, Frith CD (2006): The Kuleshov Effect: the influence of contextual framing on emotional attributions. Soc Cogn Affect Neurosci 1, 95106

Modinos G, Ormel J, Aleman A (2010): Individual differences in dispositional mindfulness and brain activity involved in reappraisal of emotion. Soc Cogn Affect Neurosci $\underline{5}$, 369- 377

Mohr C, Binkofski F, Erdmann C, Buchel C, Helmchen C (2005): The anterior cingulate cortex contains distinct areas dissociating external from self-administered painful stimulation: a parametric fMRI study. Pain $\underline{114}, 347-357$

Montgomery GH, Kirsch I (1997): Classical conditioning and the placebo effect. Pain $\underline{72,107-113}$

Morgan MA, Romanski LM, LeDoux JE (1993): Extinction of emotional learning: contribution of medial prefrontal cortex. Neurosci Lett $\underline{163}, 109-113$

Myers KM, Davis M (2002): Behavioral and neural analysis of extinction. Neuron $\underline{36}, 567-584$

Myers KM, Davis M (2007): Mechanisms of fear extinction. Mol Psychiatry $\underline{12}$, 120- 150 
Nakata H, Sakamoto K, Ferretti A, Gianni Perrucci M, Del Gratta C, Kakigi R, Luca Romani G (2008): Somato-motor inhibitory processing in humans: an event-related functional MRI study. Neuroimage $\underline{39}, 1858-1866$

Nitschke JB, Dixon GE, Sarinopoulos I, Short SJ, Cohen JD, Smith EE, Kosslyn SM, Rose RM, Davidson RJ (2006): Altering expectancy dampens neural response to aversive taste in primary taste cortex. Nat Neurosci $\underline{9}$, 435- 442

Ochsner KN, Gross JJ (2005): The cognitive control of emotion. TICS $\underline{9}$, 242- 249

Ochsner KN, Bunge SA, Gross JJ, Gabrieli JD (2002): Rethinking feelings: an FMRI study of the cognitive regulation of emotion. J Cogn Neurosci 14,1215- 1229

Ochsner KN, Ray RD, Cooper JC, Robertson ER, Chopra S, Gabrieli JD, Gross JJ (2004): For better or for worse: neural systems supporting the cognitive down- and up-regulation of negative emotion. Neuroimage 23, 483- 499

Olson IR, Plotzker A, Ezzyat Y (2007): The Enigmatic temporal pole: a review of findings on social and emotional processing. Brain $\underline{130}, 1718-1731$

O’Doherty J, Kringelbach ML, Rolls ET, Hornak J, Andrews C (2001): Abstract reward and punishment representations in the human orbitofrontal cortex. Nat Neurosci $\underline{4}, 95-102$

Onoda K, Okamoto Y, Toki S, Ueda K, Shishida K, Kinoshita A, Yoshimura S, Yamashita H, Yamawaki S (2008): Anterior cingulate cortex modulates preparatory activation during certain anticipation of negative picture. Neuropsychologia $\underline{46}, 102-110$

Orr JM, Weissman DH (2009): Anterior cingulate cortex makes 2 contributions to minimizing distraction. Cereb Cortex 19, 703- 711

Orr SP, Metzger LJ, Lasko NB, Macklin ML, Peri T, Pitman RK (2000): De novo conditioning in trauma-exposed individuals with and without posttraumatic stress disorder. J Abnorm Psychol $\underline{109}$, $290-298$

Padoa-Schioppa C, Assad JA (2006): Neurons in the orbitofrontal cortex encode economic value. Nature 441, 223- 226 
Paradiso S, Jhonson DL, Andreasen NC, O’Leary DS, Watkins GL, Ponto LL, Hichwa RD (1999): Cerebral blood flow changes associated with attribution of emotional valence to pleasant, unpleasant, and neutral visual stimuli in a PET study of normal subjects. Am J Psychiatry 156, 1618- 1629

Pavlov IP, Anrep GV (1927): Conditioned reflexes: an investigation oft the physiological activity oft the cerebral cortex; in Classics in the History of Psychology; hrsg. Green CD; York University, Toronto, Ontario; http://psychclassics.yorku.ca/Pavlov

Petrovic P, Ingvar M (2002) Imaging cognitive modulation of pain processing. Pain $\underline{95}, 1-5$

Petrovic P, Kalso E, Petersson KM, Ingvar M (2002): Placebo and opioid analgesia- imaging a shared neuronal network. Science 295, 1737- 1740

Petrovic P, Dietrich T, Fransson P, Andersson J, Carlsson K, Ingvar M (2005): Placebo in emotional processing-induced expectations of anxiety relief activate a generalized modulatory network. Neuron $\underline{46}, 957-969$

Phan KL, Fitzgerald DA, Nathan PJ, Tancer ME (2006): Association between amygdala hyperactivity to harsh faces and severity of so- cial anxiety in generalized social phobia. Biol Psychiatry $\underline{59}, 424-429$

Phelps EA (2006): Emotion and cognition: Insights from studies of the human amygdala. Annu Rev Psychol 57, 27- 53

Plassmann H, O'Doherty J, Shiv B, Rangel A (2008): Marketing actions can modulate neural representations of experienced pleasantness. Proc Natl Acad Sci U S A $\underline{105}$, 1050-1054

Ploghaus A, Tracey I, Gati JS, Clare S, Menon RS, Matthews PM, Rawlins JN (1999): Dissociating pain from its anticipation in the human brain. Science $\underline{284}$, 1979- 1981

Ploghaus A, Becerra L, Borras C, Borsook D (2003): Neural circuitry underlying pain modulation: expectation, hypnosis, placebo. Trends Cogn Sci $\underline{7}, 197-200$ 
Ploner M, Lee MC, Wiech K, Bingel U, Tracey I (2010): Prestimulus functional connectivity determines pain perception in humans. Proc Natl Acad Sci USA 107, 355-360

Porro CA, Baraldi P, Pagnoni G, Serafini M, Facchin P, Maieron M, Nichelli P (2002): Does anticipation of pain affect cortical nociceptive systems? J Neurosci $\underline{22}$, 3206- 3214

Posner MI, Rothbart MK, Sheese BE, Tang Y (2007): The anterior cingulate gyrus and the mechanism of self-regulation. Cogn Affect Behav Neurosci ㅁ, 391- 395

Price DD, Milling LS, Kirsch I, Duff A, Montgomery GH, Nicholls SS (1999): An analysis of factors that contribute to the magnitude of placebo analgesia in an experimental paradigm. Pain $\underline{83}$, 147- 156

Price DD, Finniss DG, Benedetti F (2008): A comprehensive review of the placebo effect: recent advances and current thought. Annu Rev Psychol 59, 565- 590

Quirk GJ, Beer JS (2006): Prefrontal involvement in the regulation of emotion: convergence of rat and human studies. Curr Opin Neurobiol 16, 723- 727

Quirk GJ, Russo GK, Barron JL, Lebron K (2000): The role of ventromedial prefrontal cortex in the recovery of extinguished fear. J Neurosci $\underline{20}, 6225-6231$

Quirk GJ, Likhtik E, Pelletier JG, Pare D (2003): Stimulation of medial prefrontal cortex decreases the responsiveness of central amygdala output neurons. J Neurosci $\underline{23}, 8800-8807$

Quirk GJ, Garcia R, González-Lima F (2006): Prefrontal mechanisms in extinction of conditioned fear. Biol Psychiatry $\underline{60}, 337-343$

Radulescua AR, Mujica-Parodib LR (2009): A principal component network analysis of prefrontallimbic fMRI time series in schizophrenia patients and healthy controls. Psychiatry Res $\underline{174}, 184$ 194

Ragland JD, Laird AR, Ranganath C, Blumenfeld RS, Gonzales SM, Glahn DC (2009): Prefrontal activation deficits during episodic memory in schizophrenia. Am J Psychiatry $\underline{166}, 863-874$

Rainville P (2002): Brain mechanisms of pain affect and pain modulation. Curr Opin Neurobiol $\underline{2}$, $195-204$ 
Rasia-Filho AA, Londero RG, Achaval M (2000): Functional activities of the amygdala: an overview. J Psychiatry Neurosci 25, 14- 23

Rauch SL, Shin LM, Phelps EA (2006): Neurocircuitry models of posttraumatic stress disorder and extinction: human neuroimaging research-past, present, and future. Biol Psychiatry 60, 376- 382

Ray RD, Zald DH (2012): Anatomical insights into the interaction of emotion and cognition in the prefrontal cortex. Neurosci Biobehav Rev $\underline{36}, 479-501$

Rescorla RA (1988): Pavlovian conditioning. It's not what you think. Am Psychol 43, 151- 160

Rilling ME, Neiworth JJ (1987): Theoretical and methodological considerations for the study of imagery in animals. Lern Motiv $\underline{18}, 57-79$

Robinson R, Roberts WL, Strayer J, Koopman R (2007): Empathy and emotional responsiveness in delinquent and non-delinquent adolescents. Soc Dev $\underline{16}, 555-579$

Rolls ET (2000): Memory systems in the brain. Annu Rev Psychol 51, 599- 630

Rolls ET (2007): Sensory processing in the brain related to the control of food intake. Proc Nutr Soc $\underline{66}, 96-112$

Rosenkranz JA, Moore H, Grace AA (2003): The prefrontal cortex regulates lateral amygdala neuronal plasticity and responses to previously conditioned stimuli. J Neurosci $\underline{23}, 11054-11064$

Rushworth MF, Behrens TE, Rudebeck PH, Walton ME (2007): Contrasting roles for cingulate and orbitofrontal cortex in decisions and social behaviour. Trends Cogn Sci 11, 168- 176

Sanchez-Navarro JP, Martinez-Selva JM, Roman F (2005): Emotional response in patients with frontal brain damage: effects of affective valence and information content. Behav Neurosci $\underline{119}$, $87-97$

Sander D, Grandjean D, Scherer KR (2005): A systems approach to appraisal mechanisms in emotion. Neural Netw $\underline{18}, 317-352$

Santini E, Muller R U, Quirk GJ (2001): Consolidation of extinction learning involves transfer 
from NMDA-independent to NMDA-dependent memory. J Neurosci 21, 9009- 9017

Sarinopoulos I, Dixon GE, Short SJ, Davidson RJ, Nitschke JB (2006): Brain mechanisms of expectation associated with insula and amygdala response to aversive taste: implications for placebo. Brain Behav Immun 20, 120- 132

Schacter DL, Addis DR, Buckner RL (2007): Remembering the past to imagine the future: the prospective brain. Nat Rev Neurosci $\underline{8}, 657-661$

Schiller D, Delgado MR (2010): Overlapping neural systems mediating extinction, reversal and regulation of fear. Trends Cogn Sci 14, 268- 276

Schiller D, Levy I, Niv Y, LeDoux JE, Phelps EA (2008): From fear to safety and back: reversal of fear in the human brain. J Neurosci $\underline{28}, 11517-11525$

Schmahmann JD, Caplan D (2006): Cognition, emotion and the cerebellum. Brain 129(Pt 2), 290292

Schoenbaum G, Setlow B (2001): Integrating orbitofrontalcortex into prefrontal theory: common processing themes across species and subdivisions. Learn Mem $\underline{8}, 134-147$

Scott DJ, Stohler CS, Egnatuk CM, Wang H, Koeppe RA, Zubieta JK (2008): Placebo and nocebo effects are defined by opposite opioid and dopaminergic responses. Arch Gen Psychiatry $\underline{65}, 220$ 231

Sharot T, Riccardi AM, Raio CM, Phelps EA (2007): Neural mechanisms mediating optimism bias. Nature $\underline{450}, 102-105$

Simmons A, Stein MB, Matthews SC, Feinstein JS, Paulus MP (2006): Affective ambiguity for a group recruits ventromedial prefrontal cortex. Neuroimage $\underline{29}, 655-661$

Smith EE, Jonides J (1999): Storage and executive processes in the frontal lobes. Science $\underline{283}$, $1657-1661$

Sohn MH, Albert MV, Jung K, Carter CS, Anderson JR (2007): Anticipation of conflict monitoring in the anterior cingulate cortex and the prefrontal cortex. Proc Natl Acad Sci USA 104, 1033010334 
Soliman F, Glatt CE, Bath KG, Levita L, Jones RM, Pattwell SS, Jing D, Tottenham N, Amso D, Somerville LH, Voss HU, Glover G, Ballon DJ, Liston C, Teslovich T, Van Kempen T, Lee FS, Casey BJ (2010): A genetic variant BDNF polymorphism alters extinction learning in both mouse and human. Science $\underline{327}, 863-866$

Sommer M, Döhnel K, Sodian B, Meinhardt J, Thoermer C, Hajak G (2007): Neural correlates of true and false belief reasoning. Neuroimage $\underline{35}$, 1378- 1384

Sörös P, Inamoto Y, Martin RE (2009): Functional brain imaging of swallowing: an activation likelihood estimation meta-analysis. Hum Brain Mapp 30, 2426- 1439

Sotres-Bayon F, Bush DEA, LeDoux JE (2004): Emotional preservation: an update on prefrontalamygdala interactions in fear extinction. Learn Mem 11, 525- 535

Sotres-Bayon F, Cain CK, LeDoux JE (2006): Brain Mechanisms of Fear Extinction: Historical Perspectives on the Contribution of Prefrontal Cortex. Biol Psychiatry $\underline{60}$, 329- 336

Spoormaker VI, Sturm A, Andrade KC, Schröter MS, Goya-Maldonado R, Holsboer F, Wetter TC, Sämann PG, Czisch M (2010): The neural correlates and temporal sequence of the relationship between shock exposure, disturbed sleep and impaired consolidation of fear extinction. J Psychiatr Res $\underline{44}, 1121-1128$

Spreng RN, Mar RA, Kim AS (2009): The common neural basis of autobiographical memory, prospection, navigation, theory of mind, and the default mode: a quantitative meta-analysis. J Cogn Neurosci $\underline{21}, 489-510$

Stein MB, Simmons AN, Feinstein JS, Paulus MP (2007): Increased amygdala and insula activation during emotion processing in anxiety- prone subjects. Am J Psychiatry 164, 318- 327

Summerfield JJ, Hassabis D, Maguire EA (2009): Cortical midline involvement in autobiographical memory. Neuroimage $\underline{44}$, 1188- 1200

Szameitat AJ, Lepsien J, von Cramon DY, Sterr A, Schubert T(2006): Task-order coordination in dual-task performance and the lateral prefrontal cortex: an event-related fMRI study. Psychol Res $\underline{70}, 541-552$

Talairach J, Szikla G: Atlas d'anatomie stereotaxique du telencephale: etudes anatomoradiologiques, Masson and Cie, Paris 1967 
Talairach J, Tournoux P (1988): Co-planar stereotaxic atlas of the human brain, Thieme Medical Publishers, New York 1988

Thayer RE, Newman JR, Mc Clain TM (1994): Selfregulation of mood: strategies for changing a bad mood, raising energy, and reducing tension. J Pers Soc Psychol 67, 911- 925

Tobler PN, Fiorillo CD, Schultz W (2005): Adaptive coding of reward value by dopamine neurons. Science $\underline{307}, 1642-1645$

Tremblay L, Schultz W (1999): Relative reward preference in primate orbitofrontal cortex. Nature $\underline{398}, 704-708$

Tsujimoto S, Kuwajima M, Sawaguchi T (2007): Developmental fractionation of working memory and response inhibition during childhood. Exp Psychol 54, 30-37

Turkeltaub PE, Eden GF, Jones KM, Zeffiro TA (2002): Meta-analysis of the functional neuroanatomy of single-word reading: method and validation. Neuroimage $\underline{16}, 765-780$

Urry HL, van Reekum CM, Johnstone T, Kalin NH, Thurow ME, Schaefer HS, Jackson CA, Frye CJ, Greischar LL, Alexander AL, Davidson RJ (2006): Amygdala and ventromedial prefrontal cortex are inversely coupled during regulation of negative affect and predict the diurnal pattern of cortisol secretion among older adults. J Neurosci $\underline{26}$, 4415- 4425

van den Heuvel OA, Veltman DJ, Groenewegen HJ, Witter MP, Merkelbach J, Cath DC, van Balkom AJ, van Oppen P, van Dyck R (2005): Disorder-specific neuroanatomical correlates of attentional bias in obsessive-compulsive disorder, panic disorder, and hypochondriasis. Arch Gen Psychiatry $\underline{62}, 922-933$

Vase L, Robinson ME, Verne GN, Price DD (2005): Increased placebo analgesia over time in irritable bowel syndrome (IBS) patients is associated with desire and expectation but not endogenous opioid mechanisms. Pain $\underline{115}, 338-347$

Vogt BA (2005): Pain and emotion interactions in subregions of the cingulate gyrus. Nat Rev Neurosci $\underline{6}, 533-544$

Völlm BA, Taylor ANW, Richardson P, Corcoran R, Stirling J, McKie S, Deakin JFW, Elliott R (2006): Neural correlates of theory of mind and empathy: a functional magnetic resonance imaging 
6. Literaturverzeichnis

study in a nonverbal task. NeuroImage $\underline{29}, 90-98$

Voudouris NJ, Peck CL, Coleman G (1989): Conditioned response models of placebo phenomena: further support. Pain $\underline{38}, 109-116$

Voudouris NJ, Peck CL, Coleman G (1990): The role of conditioning and verbal expectancy in the placebo response. Pain $\underline{43}, 121-128$

Vuilleumier P (2005): How brains beware: Neural mechanisms of emotional attention. Trends Cogn Sci $\underline{9}, 585-594$

Waber RL, Shiv B, Carmon Z, Ariely D (2008): Commercial features of placebo and therapeutic efficacy. JAMA 299, 1016- 1017

Wager TD (2005): The Neural Bases of Placebo Effects in Pain. Am Psychol 14,175- 179

Wager TD, Rilling JK, Smith EE, Sokolik A, Casey KL, Davidson RJ, Kosslyn SM, Rose RM, Cohen JD (2004): Placebo-induced changes in FMRI in the anticipation and experience of pain. Science $\underline{303}, 1162-1167$

Wager TD, Davidson ML, Hughes BL, Lindquist MA, Ochsner KN (2008): Prefrontal-subcortical pathways mediating successful emotion regulation. Neuron $\underline{59}$, 1037- 1050

Wager TD, Waugh CE, Lindquist M, Noll DC, Fredrickson BL, Taylor SF (2009a): Brain mediators of cardiovascular responses to social threat: part I: Reciprocal dorsal and ventral subregions of the medial prefrontal cortex and heart-rate reactivity. Neuroimage $\underline{47}, 821-835$

Wager TD, van Ast VA, Hughes BL, Davidson ML, Lindquist MA, Ochsner KN (2009b): Brain mediators of cardiovascular responses to social threat, part II: Prefrontal-subcortical pathways and relationship with anxiety. Neuroimage $\underline{47}, 836-851$

Walker E, Hernandez AV, Kattan MW (2008): Meta-analysis: Its strengths and limitations. Cleve Clin J Med 포, 431- 439

Walter H, von Kalckreuth A, Schardt D, Stephan A, Goschke T, Erk S (2009): The temporal dynamics of voluntary emotion regulation. PLoS One $\underline{4}$, e6726 
Walton ME, Mars RB (2007): Probing human and monkey anterior cingulate cortex in variable environments. Cogn Affect Behav Neurosci 7, 413- 422

Watson A, El-Deredy W, Iannetti GD, Lloyd D, Tracey I, Vogt BA, Nadeau V, Jones AK (2009): Placebo conditioning and placebo analgesia modulate a common brain network during pain anticipation and perception. Pain $\underline{145}, 24-30$

Wessa M, Flor H (2007): Failure of extinction of fear responses in posttraumatic stress disorder: evidence from second-order conditioning. Am J Psychiatry 164, 1684- 1692

Williams D, Mateer CA (1992): Developmental impact of frontal lobe injury in middle childhood. Brain and Cognition 20, 196- 204

Willoch F, Tolle TR, Wester HJ, Munz F, Petzold A, Schwaiger M, Conrad B, Bartenstein P (1999): Central pain after pontine infarction is associated with changes in opioid receptor binding: a PET study with 11C diprenorphine. Am J Neuroradiol 20, 686- 690

Winecoff A, Labar KS, Madden DJ, Cabeza R, Huettel SA (2010): Cognitive and neural contributors to emotion regulation in aging. Soc Cogn Affect Neurosci $\underline{6}, 165-176$

Wrase J, Kahnt T, Schlagenhauf F, Beck A, Cohen MX, Knutson B, Heinz A (2007): Different neural systems adjust motor behavior in response to reward and punishment. Neuroimage $\underline{36}, 1253-$ 1262

Yágüez L, Coen S, Gregory LJ, Amaro E Jr, Altman C, Brammer MJ, Bullmore ET, Williams SC, Aziz Q (2005): Brain response to visceral aversive conditioning: a functional magnetic resonance imaging study. Gastroenterology 128, 1819- 1829

Yamasue H, Kasai K, Iwanami A, Ohtani T, Yamada H, Abe O, Kuroki N, Fukuda R, Tochigi M, Furukawa S, Sada- matsu M, Sasaki T, Aoki S, Ohtomo K, Asukai N, Kato N (2004): Voxel-based analysis of MRI reveals anterior cingulate gray-matter volume reduction in posttraumatic stress disorder due to terrorism. Proc Natl Acad Sci U S A 100, 9039- 9043

Zhang W, Robertson J, Jones AC, Dieppe PA, Doherty M (2008): The placebo effect and its determinants in osteoarthritis: meta-analysis of randomised controlled trials. Ann Rheum Dis $\underline{67}$, 1716-1723 
Zubieta JK, Ketter TA, Bueller JA, Xu Y, Kilbourn MR, Young EA, Koeppe RA (2003): Regulation of human affective responses by anterior cingulate and limbic mu-opioid neurotransmission. Arch Gen Psychiatry $\underline{60}, 1145-1153$

Zubieta JK, Bueller JA, Jackson LR, Scott DJ, Xu Y, Koeppe RA, Nichols TE, Stohler CS (2005): Placebo effects mediated by endogenous opioid activity on mu-opioid receptors. J Neurosci $\underline{25}$, 7754- 7762 


\section{Abbildungsverzeichnis}

$\begin{array}{ll}\text { Abbildung } & \text { Seite }\end{array}$

Abbildung 3.1 Gehirnaktivierung bei Furchtextinktionsstudien (Rot),

Placebostudien (Blau), Emotionsregulationsstudien (Grün).

Übereinstimmende Aktivierung im VMPFC

Abbildung 3.2 Paarmetaanalyse: Verstärkte Aktivierungen in der rechten Inselrinde und dem linken ACC bei Emotionsregulations- und Placebostudien

Abbildung 3.3 Maximum der geringsten Aktivierung in der Amygdala in allen drei Studientypen

Abbildung 4.1 Abgrenzung zwischen den präfrontalen Regionen (Etkin et al. 2011, S.86)

Abbildung 4.2 Laterale und mediale präfrontale Regionen (Ray und Zald 2012) 
8. Tabellenverzeichnis

\section{Tabellenverzeichnis}

Tabelle

Seite

Tabelle 2.1 Furchtextinktionsstudien

Tabelle 2.2 Placebostudien

Tabelle 2.3 Emotionsregulationsstudien

Tabelle 3.1 ALE-Metaanalyse der Gehirnaktivierungen in Furchtextinktionsstudien 28

Tabelle 3.2 ALE-Metaanalyse der Gehirnaktivierungen in Placebostudien

Tabelle 3.3 ALE-Metaanalyse der Gehirnaktivierungen in Emotionsregulationsstudien

Tabelle 3.4 ALE-Metaanalyse der Gehirnaktivierungen in Emotionsregulationsstudien (Studien mit ähnlicher Anzahl an Männern und Frauen)

Tabelle 3.5 ALE-Metaanalyse der Gehirndeaktivierungen in allen Studientypen (Furchtextinktions-, Placebo-, Emotionsregulationsstudien)

Tabelle 8.1 ALE-Metaanalyse der Gehirndeaktivierungen in Furchtextinktionsstudien

Tabelle 8.2 ALE-Metaanalyse der Gehirndeaktivierungen in Placebostudien

Tabelle 8.3 ALE-Metaanalyse der Gehirndeaktivierungen in Emotionsregulationsstudien 


\subsection{Ergänzende Tabellen}

Tabelle 8.1: ALE-Metaanalyse der Gehirndeaktivierungen in Furchtextinktionsstudien

\begin{tabular}{|c|c|c|c|c|c|c|c|}
\hline \multirow{2}{*}{ Region } & \multicolumn{3}{|c|}{$\begin{array}{c}\text { MNI- } \\
\text { Koordinaten }\end{array}$} & \multirow{2}{*}{$\begin{array}{l}\text { ALE- } \\
\text { Wert }\end{array}$} & \multirow{2}{*}{$\begin{array}{c}\text { Clustergröße } \\
\left(\mathbf{m m}^{\wedge} \mathbf{3}\right)\end{array}$} & \multirow{2}{*}{$\begin{array}{l}\text { Anzahl der Fokusse innerhalb des } \\
\text { Clusters }\end{array}$} & \multirow{2}{*}{$\begin{array}{l}\text { Anzahl der } \\
\text { Studien }\end{array}$} \\
\hline & $\mathbf{x}$ & $\mathbf{y}$ & $\mathbf{z}$ & & & & \\
\hline L Amygdala & -26 & 0 & -26 & 0.0107 & 312 & 2 & $2 *$ \\
\hline $\begin{array}{l}\text { R frontaler } \\
\text { Gyrus }\end{array}$ & 14 & 38 & -20 & 0.0097 & 128 & 2 & 1 \\
\hline
\end{tabular}

Diese Metaanalyse beinhaltete Koordinaten von vier Studien mit 81 Probanden und insgesamt fünf Fokusse.

* Soliman et al. 2010, Spoormaker et al. 2010

Tabelle 8.2: ALE-Metaanalyse der Gehirndeaktivierungen in Placebostudien

\begin{tabular}{|c|c|c|c|c|c|c|c|}
\hline \multirow{2}{*}{ Region } & & MNI & & \multirow{2}{*}{$\begin{array}{l}\text { ALE- } \\
\text { Wert }\end{array}$} & \multirow{2}{*}{$\begin{array}{c}\text { Clustergröße } \\
\left(\mathbf{m m}^{\wedge} \mathbf{3}\right)\end{array}$} & \multirow{2}{*}{$\begin{array}{c}\text { Anzahl der Fokusse } \\
\text { innerhalb des Clusters }\end{array}$} & \multirow{2}{*}{$\begin{array}{c}\text { Anzahl der } \\
\text { Studien }\end{array}$} \\
\hline & $\mathbf{x}$ & $\mathbf{y}$ & $\mathbf{z}$ & & & & \\
\hline \multirow{3}{*}{ L Inselrinde } & -36 & 2 & -4 & 0.0170 & \multirow{3}{*}{1520} & \multirow{3}{*}{5} & \multirow{3}{*}{3} \\
\hline & & & & & & & \\
\hline & -38 & 2 & 2 & 0.0162 & & & \\
\hline R Inselrinde & 42 & 6 & $\mathbf{0}$ & 0.0158 & 488 & 2 & 2 \\
\hline & & & & & 728 & & \\
\hline $\mathrm{L} A C C$ & -2 & 30 & 20 & 0.0132 & & 4 & 3 \\
\hline L Amygdala & -24 & -2 & -18 & 0.0117 & 440 & 3 & $3 *$ \\
\hline $\begin{array}{l}\text { Fusiformer Gyrus, } \\
\text { Temporaler Lobulus }\end{array}$ & 48 & -54 & -16 & 0.0111 & 232 & 2 & 1 \\
\hline
\end{tabular}

Diese Metaanalyse beinhaltete Koordinaten von 10 Studien mit 196 Probanden und insgesamt 81 Fokusse.

* Bingel et al. 2006, Eippert et al. 2009, Petrovic et al. 2005 
Tabelle 8.3: ALE-Metaanalyse der Gehirndeaktivierungen in Emotionsregulationsstudien.

\begin{tabular}{|c|c|c|c|c|c|c|c|}
\hline \multirow{2}{*}{ Region } & & $\begin{array}{l}\text { MNI- } \\
\text { rdin }\end{array}$ & & ALE- & \multirow{2}{*}{$\begin{array}{c}\text { Clustergröße } \\
\left(\mathbf{m m}^{\wedge} \mathbf{3}\right)\end{array}$} & \multirow{2}{*}{$\begin{array}{l}\text { Anzahl der Fokusse } \\
\text { innerhalb des Clusters }\end{array}$} & \multirow{2}{*}{$\begin{array}{c}\text { Anzahl der } \\
\text { Studien }\end{array}$} \\
\hline & $\mathbf{x}$ & $\mathbf{y}$ & $\mathbf{z}$ & & & & \\
\hline \multirow{3}{*}{ L Amygdala } & -28 & -2 & -20 & 0.0273 & \multirow{3}{*}{3816} & \multirow{3}{*}{12} & \multirow{3}{*}{ 9* } \\
\hline & & & & & & & \\
\hline & -22 & 0 & -26 & 0.0170 & & & \\
\hline \multirow{3}{*}{ R Amygdala } & 22 & 0 & -26 & 0.0162 & \multirow{3}{*}{1776} & \multirow{3}{*}{8} & \multirow{3}{*}{$6^{* * *}$} \\
\hline & & & & & & & \\
\hline & 38 & 2 & -24 & 0.0118 & & & \\
\hline $\begin{array}{c}\text { L Postzentraler Gyrus, } \\
\text { Parietaler Lobulus }\end{array}$ & -58 & -24 & 24 & 0.0206 & 1064 & 4 & 3 \\
\hline \multirow{4}{*}{ L Inselrinde } & -40 & -24 & 20 & 0.0134 & \multirow{3}{*}{432} & \multirow{3}{*}{3} & \multirow{3}{*}{2} \\
\hline & & & & & & & \\
\hline & -40 & -16 & 14 & 0.0092 & & & \\
\hline & -42 & -14 & $\mathbf{0}$ & 0.0135 & 416 & 2 & 2 \\
\hline \multirow{6}{*}{ R Inselrinde } & 48 & -22 & 26 & 0.0108 & \multirow{3}{*}{216} & \multirow{3}{*}{2} & \multirow{3}{*}{2} \\
\hline & & & & & & & \\
\hline & 50 & -28 & 30 & 0.0097 & & & \\
\hline & 38 & -20 & 24 & 0.0103 & \multirow{3}{*}{136} & \multirow{3}{*}{2} & \multirow{3}{*}{2} \\
\hline & & & & & & & \\
\hline & 44 & -14 & 20 & 0.0094 & & & \\
\hline R Okzipitaler Kortex & 4 & -86 & 2 & 0.0135 & \multirow{3}{*}{312} & 2 & 1 \\
\hline \multirow[b]{2}{*}{ L Temporallappen } & & & & & & \multirow[b]{2}{*}{2} & \multirow[b]{2}{*}{2} \\
\hline & -42 & 24 & -26 & 0.0143 & & & \\
\hline L Thalamus & -24 & -28 & -2 & 0.0109 & 160 & 2 & 2 \\
\hline
\end{tabular}

Diese Metaanalyse beinhaltete Koordinaten von 15 Studien mit 280 Probanden und insgesamt 94 Fokusse.

* Delgado et al. 2008, Eippert et al. 2007, Goldin et al. 2007, Herwig et al. 2007, McRae et al. 2009, Ochsner et al. 2004, Urry et al. 2006, Walter et al. 2009, Winecoff et al. 2010

**Koenigsberg et al. 2010, McRae et al. 2009, Ochsner et al. 2004, Urry et al. 2006, Walter et al. 2009, Winecoff et al. 2010 


\section{Abkürzungsverzeichnis}

\begin{tabular}{|c|c|}
\hline ACC & Pars anterior des Gyrus cinguli \\
\hline ALE & Activation likelihood estimation \\
\hline BA & Bordmann-Areal \\
\hline CS & Konditionierter Reiz \\
\hline CR & Bedingte Reaktion \\
\hline $\mathrm{dACC}$ & Dorsaler pars anterior des Gyrus cinguli \\
\hline DLPFC & Dorsolateraler präfrontaler Kortex \\
\hline DMPFC & Dorsomedialer präfrontaler Kortex \\
\hline FDR & False discovery rate \\
\hline Fmri & Functional magnetic resonance imaging \\
\hline FP & Frontopolarer Kortex \\
\hline IAPS & International Affective Picture System \\
\hline $\mathrm{L}$ & Links \\
\hline LPFC & Lateraler präfrontaler Kortex \\
\hline MNI & Montreal Neurologisches Institut \\
\hline MRT & Magnetresonanztomographie \\
\hline NS & Neutral bedingter Reiz \\
\hline OFC & Orbitofrontaler Kortex \\
\hline PAG & Periaquäduktales Grau \\
\hline Pet & Positronen-Emissions-Tomographie \\
\hline PFC & Präfrontaler Kortex \\
\hline PTSD & Posttraumatische Belastungsstörung \\
\hline $\mathrm{R}$ & Rechts \\
\hline ROI & Regionen von besonderem Interesse \\
\hline
\end{tabular}


sgACC Subgenualer anteriorer Gyrus cinguli

UCR Unkonditionierte Reaktion

UCS Unkonditionierter Reiz

VMPFC Ventromedialer präfrontaler Kortex 


\section{Publikation zum Dissertationsthema}

Diekhof EK, Geier K, Falkai P, Gruber O (2011a): Fear is only as deep as the mind allows: a coordinate-based meta-analysis of neuroimaging studies on the regulation of negative affect. Neuroimage $\underline{58}, 275-285$ 


\section{Danksagung:}

Mein Dank gilt insbesondere meinem Doktorvater Prof. Dr. Oliver Gruber sowie meiner Betreuerin Dr. Esther Diekhof. Ihre Unterstützung, ihre wertvollen Ratschläge und Anregungen sowie die während der Anfertigung entgegengebrachte Geduld waren mir eine große Hilfe. Ferner danke ich meiner Familie und meinen Freunden für die ebenfalls tatkräftige Unterstützung während der Entstehung. 